\title{
Mapping the nonlinear crack tip deformation field in soft elastomer with a particle tracking method
}

\author{
Yuan $\mathrm{Qi}^{1}$, Zhanan Zou ${ }^{1}$, Jianliang Xiao ${ }^{1}$, Rong Long ${ }^{1 *}$ \\ ${ }^{1}$ Department of Mechanical Engineering, University of Colorado Boulder, Boulder, CO \\ 80309 \\ *Corresponding author: Rong.Long@,colorado.edu
}

\begin{abstract}
Stretchable and fracture-resistant elastomers or gels are highly desirable in a wide range of existing and emerging applications. Fracture of soft materials, especially the ones with significantly enhanced toughness, typically involves severe deformation near the crack tip. However, experimental characterization of the crack tip deformation field in soft materials has been challenging due to its highly non-uniform and nonlinear nature. This is an important limitation towards a complete understanding of the intricate mechanics involved in soft material fracture. In this paper we present experimental data of the nonlinear, multiaxial crack tip deformation field obtained by tracking randomly distributed tracer particles. Specifically, we measured the fields of displacement and deformation gradient around the tip of a Mode-I crack in a soft silicone elastomer. We experimentally observed, for the first time, a region of validity for the asymptotic solution of crack tip deformation field in hyperelastic solids with strong strain stiffening. Furthermore, the measured crack tip deformation field enables local evaluation of energy release rate through the J-integral, which can remove the requirement of specific experimental geometries, such as the pure shear fracture test, to measure the fracture toughness. It also enables determination of the true crack extension length defined in the reference configuration. Based on these two capabilities, we were able to measure the crack growth resistance behavior and the ratedependence of fracture toughness for soft materials with highly blunted cracks.
\end{abstract}

Keywords: fracture mechanics, particle tracking, large deformation, energy release rate. 


\section{Introduction}

Soft polymeric materials, with modulus on the order of $\mathrm{kPa}$ to $\mathrm{MPa}$, underlie many industrial applications from sealants to pressure sensitive adhesives. Recent advancements in polymer engineering have led to soft elastomers or gels with remarkable new properties such as stimuli-responsiveness ${ }^{1,2}$ and biocompatibility ${ }^{3}$. These new soft materials have enabled a wide range of emerging technologies including soft robotics ${ }^{4-6}$, biomedical implants $^{7}$ and stretchable electronics ${ }^{8-10}$. In most of these applications, the soft material needs to be stretchable to achieve functionality and yet resistant to fracture to maintain reliability. The capability of a material to resist fracture is measured by the toughness $\Gamma$ (unit: $\mathrm{J} / \mathrm{m}^{2}$ ), which quantifies the work required to grow a crack by a unit area and is widely used for evaluating a material's brittleness or defect-sensitivity ${ }^{11,12}$.

Various physical or chemical mechanisms have been developed to enhance the toughness of soft materials ${ }^{13-17}$. These mechanisms share a common principle: to introduce energy dissipation into the polymer network ${ }^{18}$. Following this principle, soft materials that are ultra-stretchable and yet mechanically robust have become reality ${ }^{14-17}$. Although it has been demonstrated that dissipation can lead to a dramatic increase in fracture toughness by orders of magnitude ${ }^{18}$, quantitative principles connecting dissipation and toughness are still missing ${ }^{12}$. A major challenge is that dissipation is closely coupled to the highly nonuniform deformation field near the crack tip ${ }^{19,20}$, which makes it extremely difficult to accurately quantify the amount of energy dissipation. Therefore, an in-depth understanding of the crack tip deformation field in soft materials is necessary for establishing predictive fracture models and quantitative guidelines to engineer soft functional materials with high toughness ${ }^{21,22}$. Indeed, the asymptotic solutions of crack tip stress and strain fields have served as a fundamental pillar supporting the development of classical fracture mechanics ${ }^{23}$. However, solutions based on linear elasticity have been deemed inadequate for soft materials due to nonlinearities associated with large deformation ${ }^{19}$. Analytical or computational approaches to reveal the crack tip field in soft materials are challenging due to incomplete understandings in both the multi-axial constitutive relations under large deformation $^{24,25}$ and the local failure process at crack tip ${ }^{21}$, especially for soft materials with strong dissipative behaviors ${ }^{12}$. As a result, existing crack tip field solutions for soft 
materials have been limited to a few nonlinear elastic models ${ }^{20}$. Nonlinear crack tip field solutions accounting for more complex bulk material behaviors, such as viscoelasticity and Mullins effect, are not yet available. Therefore, experimental measurement of the crack tip deformation field in soft materials becomes especially valuable. More specifically, it can provide critical inputs in at least three aspects described below, which will be addressed in this work.

- Determining region of validity for asymptotic crack tip solutions. Analytical solutions for the nonlinear crack tip field can provide useful theoretical insights on the fracture process $^{20}$. These solutions are asymptotic in nature and thus are only valid in the vicinity of the crack tip. However, in reality the hyperelastic assumption underlying these asymptotic solutions may break down within the fracture process zone at the crack tip ${ }^{12}$. Therefore, whether there exists a region of validity for such asymptotic solutions is unclear, which can be answered by comparing the experimental measured crack tip field with the asymptotic solutions.

- Enabling local evaluation of energy release rate. Current methods to measure the fracture toughness of soft materials are limited to only a few testing geometries ${ }^{26}$ for which the energy release rate $G$ can be calculated through a global energy balance. If the crack tip deformation field can be measured experimentally, one could also use the J-integral ${ }^{23,27,28}$ to evaluate $G$ provided that the material is elastic (except in the vicinity of the crack tip). This approach can greatly expand the capability of current fracture tests by removing the limitation on testing geometry and accommodating more complex loading conditions.

- Decoupling crack extension and large deformation. Large deformation can cause significant blunting of a sharp crack tip ${ }^{29,30}$, which may displace the crack tip even if the crack has not propagated yet. This effect makes it difficult to determine the onset of crack propagation by monitoring the crack tip position in the deformed configuration. The measured crack tip deformation field would allow us to map the crack tip position back to the reference configuration and to define crack extension in the reference configuration, thereby decoupling crack extension and large deformation. 
Although important, experimental measurement of the crack tip field in soft materials has been difficult due to the large deformation and severe strain gradients near the crack tip ${ }^{29,31}$. Digital image correlation ${ }^{32}$ (DIC) has been widely used for in situ measurement of the displacement and strain fields. By correlating sub-elements in the digital images of a material sample taken before and after deformation based on the grayscale pattern ${ }^{32}$, the DIC method is able to accurately measure the displacement field with sub-pixel resolution ${ }^{32-34}$. Large deformation poses a challenge to the DIC method, since the sub-element to be correlated may be subjected to significant stretch and rotation ${ }^{35}$, which are not known a priori but need to be considered when implementing the correlation scheme. This problem has been addressed either by using advanced algorithms to correct the distortion of correlation elements ${ }^{35-37}$ or by dividing the deformation history into small increments $^{38,39}$. Indeed, numerous works in the literature have applied the DIC method to soft elastomers ${ }^{40-42}$, gels ${ }^{21,43}$, biological tissues ${ }^{44}$, or thermoplastic polymers with large plastic deformation ${ }^{45}$. Despite these applications of the DIC method for large deformation, very few works have used it to measure the nonlinear crack tip deformation field in soft materials. For example, Livne et al. ${ }^{19}$ measured the near-tip deformation field for Mode-I dynamic cracks to demonstrate the effect of nonlinear deformation, but this work focused on brittle gels with moderate deformation around the crack tip (e.g., tensile strain up to $\sim 20 \%$ ). Crack tip fields with large strains, i.e., strains up to a few hundred percent, have also been characterized by the DIC method, e.g., in Mzabi et al. ${ }^{46}$ who studied fatigue crack propagation in filled elastomers, or in Zhang et al. ${ }^{21}$ who studied the toughening mechanism of an interpenetrating network gel. However, these works did not discuss quantitative details of the measured crack tip deformation field. Systematic experimental investigations of the nonlinear, multi-axial crack tip deformation field in soft materials are still lacking in the literature.

In this paper, we adopt an experimental method ${ }^{47,48}$ different from DIC to measure the nonlinear crack tip deformation field in soft elastomers, specifically by tracking the displacements of randomly distributed tracer particles deposited on the surface of the fracture sample. Because the tracking is performed discretely for individual tracer particles, this particle tracking method is insensitive to distortions caused by large deformation and is suitable for problems with displacement discontinuity (e.g., opening of a sharp crack). 
These advantage of the particle tracking method is analogous to that of the element-free method ${ }^{49}$ in computation mechanics. The paper is organized as follows. Section 2 describes the experimental procedures and data processing of our particle tracking method. In Section 3, we apply this method to a soft silicone elastomer and show for the first time that the asymptotic crack tip solutions for hyperelastic solids with strain stiffening can be observed experimentally. In Section 4, we demonstrate that for predominantly elastic materials, the measured crack tip deformation field can be used to evaluate the energy release rate through the path-independent J-integral. We are also able to identify the true crack extension length defined in the reference configuration, which further allows us to determine crack growth resistance behavior and rate-dependence of fracture toughness. Conclusions are given in Section 5.

\section{Particle tracking method}

\subsection{Overview}

The working principle of the particle tracking method is illustrated using an example shown in Fig.1. An initially sharp crack in a thin polydimethylsiloxane (PDMS) elastomer sample is subjected to an increasing tensile displacement $\Delta$ perpendicular to the crack. We will use the initial undeformed configuration as the reference configuration throughout this paper. To measure the deformation field, we deposited randomly distributed tracer particles on the surface of the sample. The tracer particles must be much thinner than the sample, such that they can be well attached to the sample and do not affect its deformation. For the example shown in Fig.1a, we used glitter flakes $(0.2 \sim 0.3 \mathrm{~mm}$ in diameter) as the tracer particles. Multiple images of the sample and tracer particles were taken along the loading history and then fed into a custom developed image processing program to locate the two dimensional (2D) coordinates $\mathbf{x}$ of the centroid of each tracer particle. The same particle appearing in different images were then tracked and linked along the loading history. The tracking procedure generated a history of displacement $\mathbf{u}$ for each particle (see Fig. 1b):

$$
\mathbf{u}(\mathbf{X}, t)=\mathbf{x}(\mathbf{X}, t)-\mathbf{X}
$$


where $\mathbf{X}$ is the initial coordinate vector of a tracer particle and $\mathbf{x}$ is the corresponding coordinate vector at time $t$. Finally, the strain field (see Fig.1c) can be obtained by interpolating the discrete displacement data into a continuous displacement field and then calculating the spatial gradient of the interpolated displacement field.

The three main components of the particle tracking method, i.e., 1) sample preparation, mechanical loading and in situ imaging; 2) image processing and particle tracking; 3) interpolation and calculation of the strain field, will be described in detail in Sections 2.2-2.4, respectively.

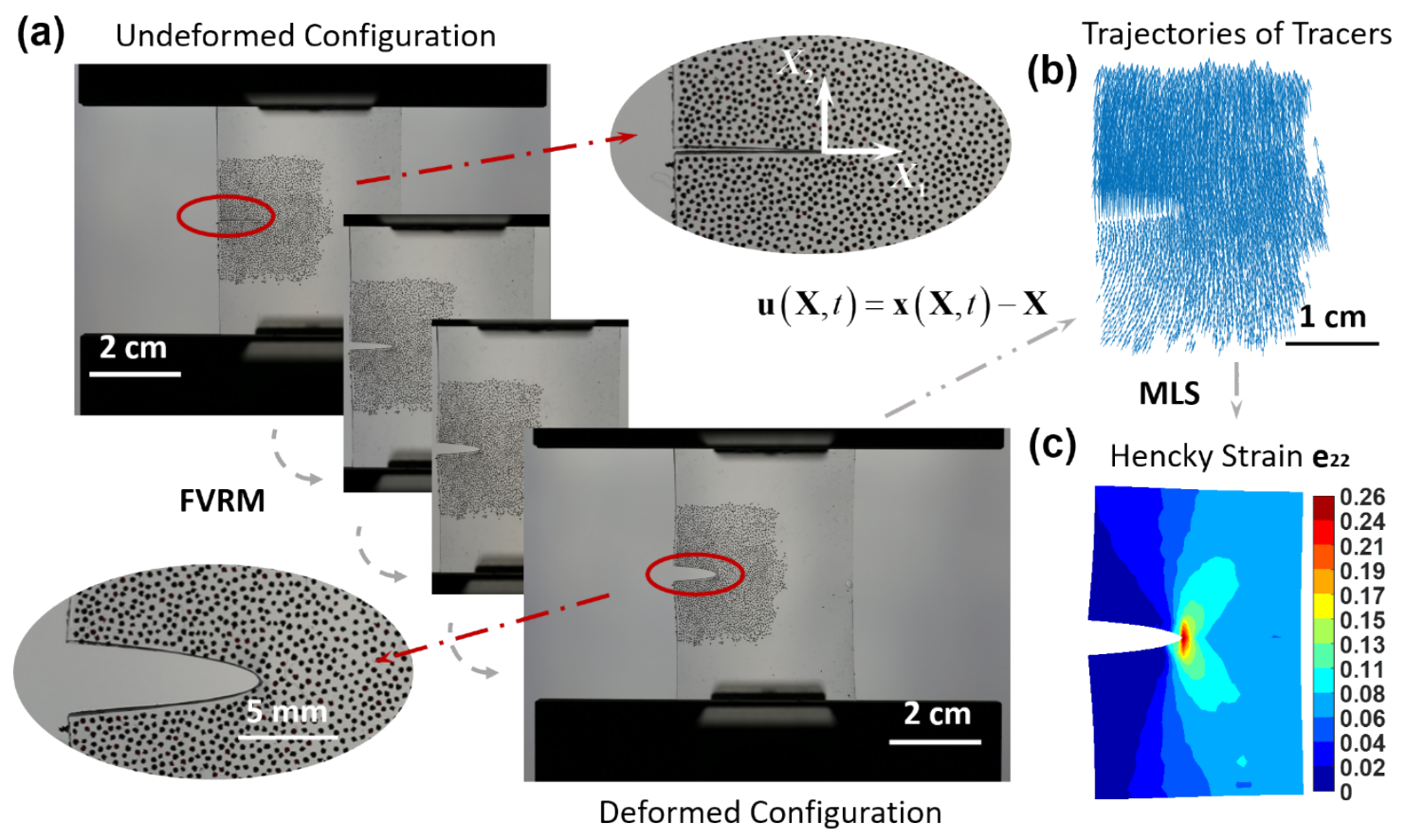

Figure 1 Overview of the particle tracking method. (a) Deformation history of the crack in a tensile sample (dimensions: height $H_{0}=50 \mathrm{~mm}$, width $L_{0}=40 \mathrm{~mm}$, thickness $T_{0}=1 \mathrm{~mm}$ ). The tracer particles are glitter flakes adhered to the sample surface. Images were taken every 5 seconds at a strain rate of $\dot{\Delta} / H_{0}=10^{-3} \mathrm{~s}^{-1}$. (b) Displacement vectors tracked for 2450 tracer particles. (c) Experimentally measured color map for the Hencky strain component $e_{22}$ at an applied loading of $\Delta / H_{0}=9.5 \%$.

\subsection{Sample preparation, fracture test and imaging}

The PDMS sample in Fig.1 was fabricated by first mixing base polymer and curing agent with a 10 to 1 ratio. After degassing for 45 minutes, the mixture was poured into a 
mold and placed on a flat table overnight. After curing, a crack was introduced at the edge of the PDMS sheet by a scalpel. Then glitter flakes (Signature ${ }^{\mathrm{TM}}$ Extra Fine Glitter by Recollections ${ }^{\mathrm{TM}}$ ), which served as tracer particles (diameter: $0.2 \sim 0.3 \mathrm{~mm}$ ), were manually placed on the surface of the sample with a tweezer.

The sample was mounted on a mechanical testing machine (Instron 5965) with the top and bottom edges clamped. A displacement loading was applied at the upper edge with a fixed velocity at $0.05 \mathrm{~mm} / \mathrm{s}$, i.e. strain rate $\dot{\Delta} / H_{0}=10^{-3} \mathrm{~s}^{-1}$. Meanwhile, deformation of the sample was monitored via a camera (Canon EOS 6D DSLR along with Canon 100mm F/2.8L Macro Lens). To enhance the optical contrast between the tracer particles and the background, a white light source and the camera were placed on the two opposite sides of the sample, so that the light emitted from the light source transmitted through the sample and was then collected by the camera. Since the PDMS sample was transparent while the tracer particles were not, the tracer particles appeared as black dots in the images (e.g., see Fig.1a). Imaging rate of the camera was set to be 0.1 frame per second (fps) before the onset of crack propagation, and was increased to $0.2 \mathrm{fps}$ afterwards.

\subsection{Image processing and particle tracking}

For each of the image frames taken during the experiment, the centroids of individual tracer particles were extracted by first binarizing the images and then using the built-in centroid finding function in MATLAB (MathWorks). To identify the same particle in consecutive images, we adopted the adaptive feature vector based relaxation (FVRM) tracking algorithm ${ }^{50}$ among others ${ }^{51,52}$. This algorithm takes advantage of the randomness of tracer particle distribution and uses the relative positions of neighboring particles as a unique geometric signature for one particle. When implementing this algorithm, we need to keep the deformation between two consecutive time frames small so that the geometry signature of neighboring particle is not severely distorted. After evaluating the matching likelihood between the target particle and a few candidate particles in the next time frame based on the geometric signature, the candidate particle with the highest likelihood that is also larger than a prescribed threshold is taken as the matching particle. This tracking algorithm was able to identify trajectories for nearly all the tracer particles, while exception may occur near the crack tip since crack propagation may break the local tracer particle 
pattern and thus the geometric signature required by the tracking algorithm is lost. In the latter case, we developed a program to manually match tracer particles between consecutive time frames. The number of particles that had to manually matched are less than $1 \%$ of the total particles.

\subsection{Interpolation and strain field}

The particle tracking process provides the coordinates of individual tracer particles at different time frames. The displacement $u_{i}(i=1,2)$ for the tracked particles can be derived from the initial coordinates (or the reference coordinates) $X_{i}$ and the deformed coordinates $x_{i}$ using eq.(1). The spatial resolution of our method to measure the particle displacement is estimated to be on the order of 1 pixel ( $~ 8 \mu \mathrm{m}$ in our images) due to the centroid finding function, which is lower than the sub-pixel resolution of the DIC method. However, since we focus on large deformation where the particle displacements are much larger than 1 pixel, the spatial resolution of our method turned out to be sufficient for mapping the crack tip displacement field.

To obtain the strain field, the displacements measured at discrete tracer points must be converted into a continuous field so that gradients can be evaluated. Since the tracer particles in our method are randomly distributed, we adopted the moving least square (MLS) scheme $^{49}$ capable of accommodating complex fields with simple polynomial basis ${ }^{48}$. The MLS method constructs a generic interpolation field function $v(\mathbf{X})$ using a polynomial basis $\mathbf{P}(\mathbf{X})$ (column vector) and the corresponding coefficients $\mathbf{a}(\mathbf{X})$ (column vector) as follows:

$$
v(\mathbf{X})=\mathbf{P}^{\mathrm{T}}(\mathbf{X}) \mathbf{a}(\mathbf{X})
$$

Unlike conventional interpolation methods where the coefficient vector $\mathbf{a}$ is constant, in MLS $\mathbf{a}(\mathbf{X})$ is position-dependent and is determined by minimizing a weighted least-square error function defined as:

$$
L=\sum_{k=1}^{m} q\left(\mathbf{X}-\mathbf{d}_{k}\right)\left[\mathbf{P}^{\mathbf{T}}\left(\mathbf{d}_{k}\right) \mathbf{a}(\mathbf{X})-w_{k}\right]^{2}
$$


where $q\left(\mathbf{X}-\mathbf{d}_{k}\right)$ is a weight function, $w_{k}$ is the measured data at the tracer particle located at $\mathbf{d}_{k}$ (in terms of reference coordinates) and $m$ is the number of tracer particles. There are many possible selections for the polynomial basis and the weight function, and effects of these functions has been studied in a previous work ${ }^{48}$. Here we adopt the simplest form for the polynomial basis $\mathbf{P}(\mathbf{X})=\left[1, X_{1}, X_{2}\right]^{T}$ and an exponential weight function as:

$$
q\left(\mathbf{X}-\mathbf{d}_{k}\right)= \begin{cases}\frac{\exp \left(1-\left|\mathbf{X}-\mathbf{d}_{k}\right|^{2} / r_{c}^{2}\right)-1}{e-1}, & \left|\mathbf{X}-\mathbf{d}_{k}\right| \leq r_{c} \\ 0 & ,\left|\mathbf{X}-\mathbf{d}_{k}\right|>r_{c}\end{cases}
$$

where $r_{c}$ is a cut-off radius beyond which the weight function is zero. Applying this interpolation scheme to each displacement component $u_{i}(i=1,2)$ gives a continuous displacement field $\mathbf{u}(\mathbf{X})$. Once the displacement field is obtained, the deformation gradient tensor $\mathbf{F}(\mathbf{X})$ can be obtained by taking gradient of the displacement field as $\mathbf{F}(\mathbf{X})=\nabla_{\mathbf{X}} \mathbf{u}(\mathbf{X})+\boldsymbol{\delta}$ where $\boldsymbol{\delta}$ is the identity tensor, i.e., $\delta_{i j}=1$ only when $i=j$. Then strain tensors such as the Green strain tensor $\mathbf{E}_{\mathrm{G}}=\left(\mathbf{F}^{T} \mathbf{F}-\boldsymbol{\delta}\right) / 2$ can be calculated using the deformation gradient tensor $\mathbf{F}$. The strain field measured with this method does not rely on any assumptions on the material's constitutive behavior.

For the example in Fig.1, we evaluated the strain fields in terms of the Hencky strain. A representative field of the Hencky strain component $e_{22}$ around the crack tip is shown in Fig.1c, where the maximum strain is found to be $26 \%$. Note that the maximum strain captured in the experimental data may depend on how close our method can approach the crack tip, which is limited by the finite size of the tracer particles $(\sim 0.3 \mathrm{~mm})$. Because the strain fields are highly concentrated towards the crack tip, it is desirable to place more tracer particles in the vicinity of the crack tip to better resolve the strain fields. This would require tracer particles with a smaller size.

\section{Nonlinear crack tip deformation field in a soft elastomer}

\subsection{Experiment}


The PDMS elastomer shown in Fig. 1 is brittle. As a result, the crack cannot sustain much deformation before it starts to propagate. In tough gels and elastomers, the crack can become severely blunted before propagation ${ }^{14-17}$. To achieve this scenario, we adopted a more stretchable silicone elastomer (Ecoflex $®$ ), often used to fabricate soft robotic devices ${ }^{53}$, for the fracture test. In addition, to deposit more tracer particles near the crack tip, we used smaller tracer particles (diameter $\sim 0.1 \mathrm{~mm}$ ) fabricated by photo lithography and transfer printing. The thickness of the tracer particles $(\sim 1 \mu \mathrm{m})$ was a thousand times smaller than that of the Ecoflex elastomer sample $(\sim 1 \mathrm{~mm})$. A detailed description of the sample preparation process is included in Appendix 1.

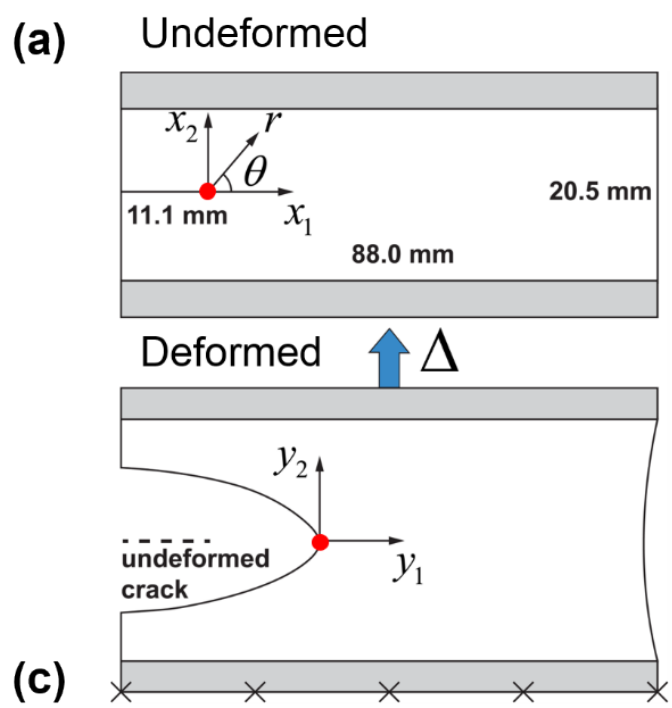

(b)
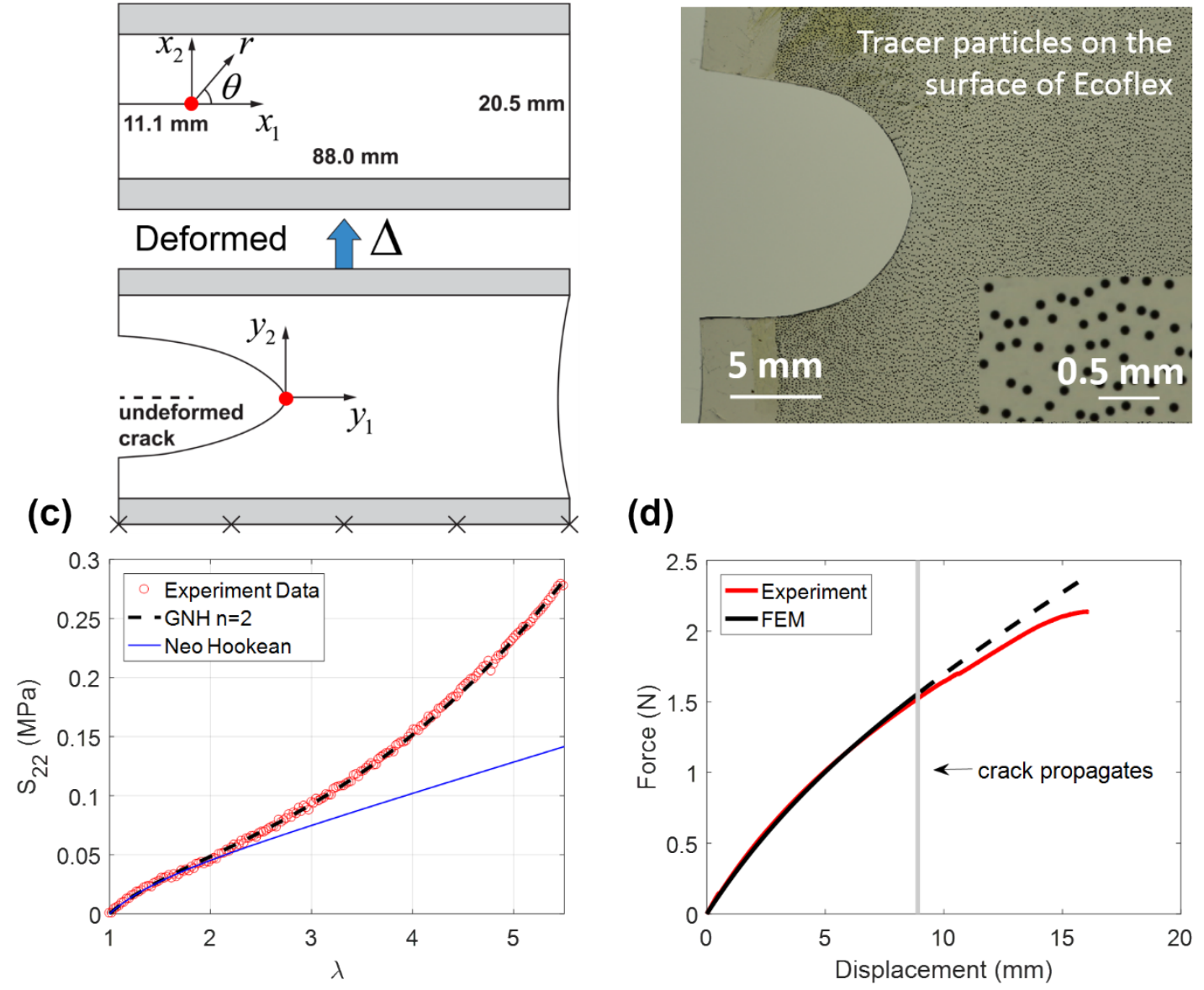

(e)

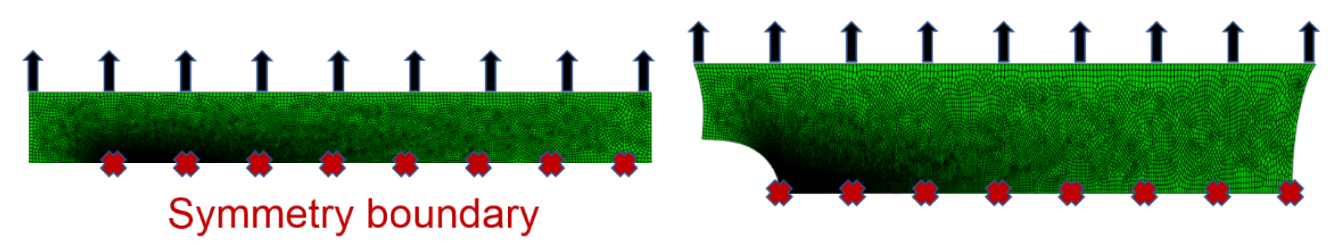


Figure 2 Fracture test of the Ecoflex elastomer. (a) Undeformed and deformed geometry of the pure shear crack sample. (b) A representative image showing the crack deformation profile and tracer particles (c) Uniaxial tension data of an Ecoflex elastomer (strain rate = $5 \times 10^{-3} \mathrm{~s}^{-1}$ ): nominal tensile stress $S_{22}$ versus the stretch ratio $\lambda$. The experimental data can be well fitted by the generalized neo-Hookean (GNH) model with $n=2$. For comparison, the curve given by the neo-Hookean model with the same shear modulus $\mu$ is also plotted as the solid line. (d) The experimentally measured force-displacement curve for the fracture test and the results predicted by the finite element method (FEM) where the elastomer was modeled as a GNH hyperelastic solid with parameters $\mu=20 \mathrm{kPa}, b=0.0626$, and $n=2$. (e) Geometry and boundary conditions of the FEM simulation.

The pure shear geometry ${ }^{54}$, which refers to a thin sheet with width that is much larger than the height ${ }^{55}$, was adopted for the fracture test (see Fig.2a). Specifically, dimensions of the sample are: width $L_{0}=88.0 \mathrm{~mm}$, height $H_{0}=20.5 \mathrm{~mm}$ and thickness $T_{0}=0.9 \mathrm{~mm}$. The initial crack length $c_{0}=11.1 \mathrm{~mm}$ is much smaller than the sample width $L_{0}$. The sample was subjected to Mode-I loading in terms of a tensile displacement $\Delta$ at a fixed velocity of $0.01 \mathrm{~mm} / \mathrm{s}$ (see Fig.2a). The applied tensile loading is quantified by the ratio $\Delta / H_{0}$, i.e., the nominal tensile strain far ahead of the crack tip. Images of the sample were taken following the same approach described in Section 2.2. Figure $2 \mathrm{~b}$ shows an example image of the deformed crack and tracer particles. Next we applied the same procedures of image processing, particle tracking and interpolation outlined in Sections 2.3 and 2.4. To increase the efficiency of image processing, here we took advantage of the circular shape of the tracer particles (see inset of Fig.2b) and used the built-in imfindcircles function in MATLAB (MathWorks) to locate the centroid of each tracer particle. This set of data processing procedures yielded a history of the displacement and deformation fields surrounding the crack tip.

A constitutive model for the Ecoflex elastomer is needed to compare the experimentally measured deformation field with theoretical or numerical solutions. Motivated by this need, we performed independent uniaxial tensile tests (Instron 5965) under a fixed strain rate $\left(5 \times 10^{-3} \mathrm{~s}^{-1}\right)$ which was chosen to match nominal strain rate of the fracture example $\dot{\Delta} / H_{0}$. Sample-to-sample variations in the tensile data were observed, especially at high stretch ratios where the strain stiffening effect starts to emerge. A representative nominal stress versus stretch ratio curve is shown in Fig.2c. The tensile data 
can be well described by the generalized neo-Hookean (GNH) model ${ }^{56}$ with the following strain energy density function:

$$
W=\frac{\mu}{2 b}\left\{\left[1+\frac{b}{n}\left(I_{1}-3\right)\right]^{n}-1\right\},
$$

where $\mu$ is the shear modulus at infinitesimal strain and $I_{1}$ is the sum of the squares of three principal stretches $\lambda_{\mathrm{i}}(i=1,2,3)$. The exponent $n$ controls the degree of strain stiffening, and the dimensionless parameter $b$ controls the onset of strain stiffening. The neo-Hookean model can be recovered from eq.(5) when $n=1$. All of our tensile data for different Ecoflex samples can be well fitted by eq. (5) with $n=2$, indicating a strong strain stiffening behavior, while $\mu$ and $b$ exhibit sample-to-sample variation ( $\mu=20 \sim 27 \mathrm{kPa}$ and $b=0.06 \sim 0.2)$. Separate tensile tests were carried out for each fracture sample to determine the corresponding material parameters $\mu$ and $b$. Cyclic tensile tests were also carried out to confirm that the Ecoflex elastomer can be modeled as an elastic solid for interpreting the measured crack tip field. Detailed discussions are included in Appendix 2.

To validate the constitutive model, we use the finite element method (FEM) to simulate deformation of the fracture sample in a commercial software ABAQUS (Dassault Systèmes). Specifically, due to symmetry of the Mode-I condition, we only included the top half of the pure shear sample in the model (see Fig.2e). The boundary at the bottom was divided into two parts, with one part being the traction free crack surface and the other part (ahead of the crack tip) with zero vertical displacement $u_{2}=0$. Therefore, the model can only simulate crack deformation but not crack propagation. Dimensions and loading conditions of this model are set to be equivalent to the experiments (see Fig.2e). We implemented a user subroutine UHYPER to incorporate the GNH model with $n=2, \mu$ $=20 \mathrm{kPa}$, and $b=0.0626$ according to the uniaxial tensile data in Fig. $2 \mathrm{c}$. The model was meshed into 47505 CPS4 elements with the smallest element size being $10^{-3} \mathrm{~mm}$. As shown in Fig.2d, the force-displacement curve predicted by FEM agrees well with the experimental data until the crack starts to propagate, which supports the validity of the constitutive model. The deviation after the onset of crack propagation is due to the fact that the FEM model was not equipped with the capability of simulating crack propagation. 


\subsection{Asymptotic solution}

The crack in our fracture experiment is subjected to the plane stress Mode-I condition, for which the asymptotic solution of crack tip deformation field using the GNH model has been obtained in the literature ${ }^{57,58}$. Here we briefly summarize the results. The solution relates the deformed coordinates $x_{i}(i=1,2)$ of a material point to its reference coordinates $X_{j}(j=1,2)$. In the asymptotic analysis, the polar coordinates $r$ and $\theta$ in the reference configuration are used instead of $X_{j}: X_{1}=r \cos \theta$ and $X_{2}=r \sin \theta$ (see Fig.2a). Also, in the deformed configuration, we use the coordinates $y_{i}$ centered at the deformed crack tip which has displaced from its original position. Therefore, $y_{i}$ differs from $x_{i}$ (centered at the undeformed crack tip) uniformly by the crack tip displacement. The first order asymptotic solution, expressed using $y_{1}$ and $y_{2}$ as functions of the reference polar coordinates $r$ and $\theta$, depends on the strain stiffening exponent $n$, but not on $\mu$ and $b$. As discussed in Section 3.2, the Ecoflex elastomer used in our experiments was found to be well described by the GNH model with $n=2$. In this case, the first order asymptotic solution is $^{57,58}$ :

$$
y_{1}=A^{-\frac{1}{2}} r^{\frac{9}{8}} g(\theta), \quad y_{2}=A r^{\frac{3}{4}} f(\theta)
$$

where $A$ is an undetermined coefficient that depends on the remote loading. The angular function $f(\theta)$ is

$$
f(\theta)=[2 \omega+\cos \theta]^{\frac{1}{4}} \sin \frac{\theta}{2}\left[1-\frac{\cos ^{2}(\theta / 2)}{2+2 \omega}\right]^{1 / 2}
$$

where

$$
\omega=\sqrt{1-\frac{\sin ^{2} \theta}{4}}
$$

On the other hand, the angular function $g(\theta)$ can only be solved numerically from the following nonlinear ordinary differential equation ${ }^{58}$ : 
$8\left(\xi^{4}+\frac{27}{16} f^{2}\right) g^{\prime \prime}=-\frac{45}{8} g \xi^{4}-f^{\prime} \xi+\frac{27}{4} f\left(f^{\prime} g^{\prime}+3 f^{\prime \prime} g\right)-\frac{9 f f^{\prime}+16 f f^{\prime \prime}}{9 f^{2}+16\left(f^{\prime}\right)^{2}}\left(16 g^{\prime} \xi^{4}+12 f \xi\right)$,

where

$$
\xi=\frac{9}{8} f^{\prime} g-\frac{3}{4} f g^{\prime}
$$

and the superscript ' denotes the first order derivative and " denotes the second order derivative with respect to $\theta$. The boundary conditions for eq.(9) is

$$
g^{\prime}(\theta=0)=0 \quad \text { and } g^{\prime}(\theta=\pi)=-\sqrt{4 / 3} \text {. }
$$

The angular functions $f(\theta)$ and $g(\theta)$ are plotted in Fig.3.

(a)

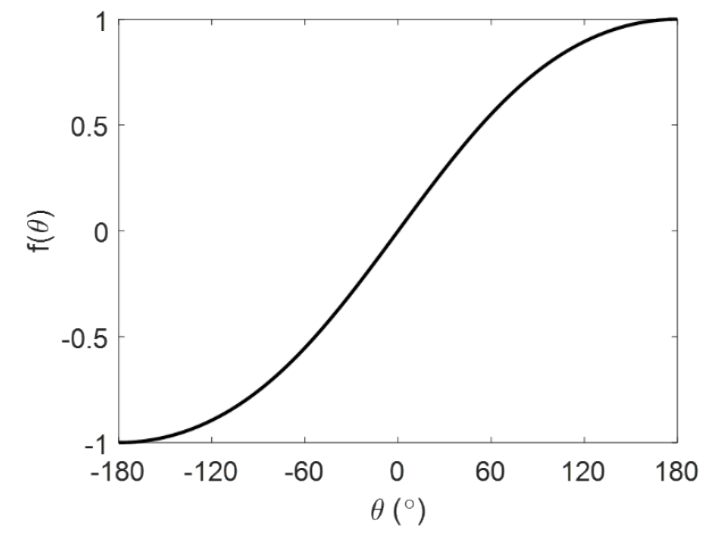

(b)

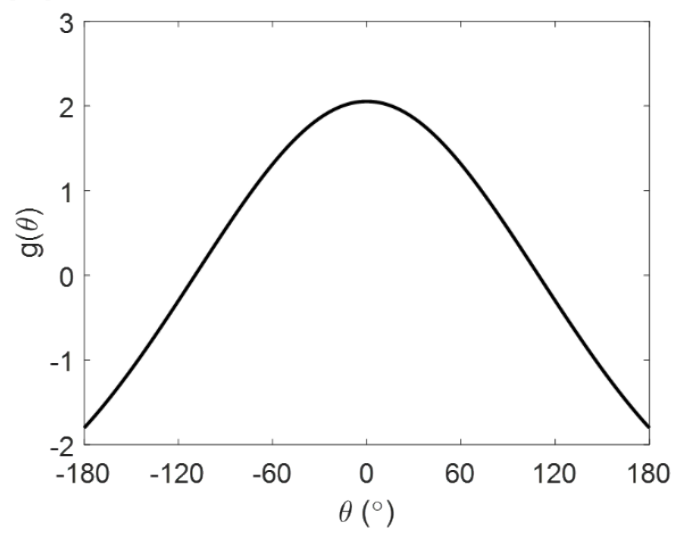

Figure 3 Angular functions in the asymptotic solution. (a) $f(\theta)$ given by eq.(7). (b) $g(\theta)$ obtained by numerically solving eq.(9) with boundary conditions in eq.(11).

The deformation gradient tensor $\mathbf{F}$ can be obtained using

$$
F_{i j}=\frac{\partial y_{i}}{\partial X_{j}}, \quad i, j=1 \text { or } 2
$$

Using $X_{1}=r \cos \theta$ and $X_{2}=r \sin \theta$, the following first order asymptotic solution for $F_{i j}$ can be derived:

$$
F_{11}=A^{-1 / 2} r^{\frac{1}{8}}\left(\frac{9}{8} \cos \theta g(\theta)-\sin \theta g^{\prime}(\theta)\right)
$$




$$
\begin{gathered}
F_{12}=A^{-1 / 2} r^{\frac{1}{8}}\left(\frac{9}{8} \sin \theta g(\theta)+\cos \theta g^{\prime}(\theta)\right) \\
F_{21}=\frac{A}{4} r^{-\frac{1}{4}}\left(3 \sin (\theta / 2) \cos \theta \sqrt{1-\frac{\cos ^{2}(\theta / 2)}{2+2 \omega}}-\sqrt{\frac{3}{2}} \sin \theta \sqrt{\omega+\cos \theta}\right)(2 \omega+\cos \theta)^{1 / 4}, \\
F_{22}=\frac{A}{4} r^{-\frac{1}{4}}\left(3 \sin (\theta / 2) \sin \theta \sqrt{1-\frac{\cos ^{2}(\theta / 2)}{2+2 \omega}}+\sqrt{\frac{3}{2}} \cos \theta \sqrt{\omega+\cos \theta}\right)(2 \omega+\cos \theta)^{1 / 4} .
\end{gathered}
$$

It should be emphasized that the crack tip deformation field solution summarized above is asymptotic in nature and thus is only valid as $r$ approaches 0 . The region of validity for this asymptotic solution is unknown and will be discussed in Section 3.3 and 3.4.

\subsection{Nonlinear crack tip deformation field}

The experimentally measured crack tip deformation field will be quantitatively examined in this section. Since our data includes a series of loading steps with increasing remote loading $\Delta / H_{0}$, we choose the step where $\Delta / H_{0}=68.78 \%$ as a representative example. At this level of loading, the crack tip became significantly blunted (see Fig.5), which is an indication of large deformation in the crack tip region.

We first focus on the mapping from the reference polar coordinates $(r, \theta)$ to the deformed coordinates $\left(y_{1}, y_{2}\right)$ as defined in Fig.2a. Using the particle tracking method, we experimentally extracted the dependence of $y_{1}$ and $y_{2}$ on $r$ and $\theta$, as shown in Fig.4. Specifically, the radial dependence of $y_{1}$ and $y_{2}$ with fixed angles $\theta$ is shown in Figs.4a and $4 \mathrm{~b}$, respectively, while Figs. $4 \mathrm{c}$ and $4 \mathrm{~d}$ plot the angular dependence of $y_{1}$ and $y_{2}$ with fixed radii $r$. To check the accuracy of our experimental results, we also included the $y_{1}$ and $y_{2}$ predicted by FEM subjected to the same remote loading $\Delta / H_{0}=68.78 \%$ (solid lines) which agree with the experimental results perfectly. This agreement shows that our particle tracking method can accurately measure the displacement field near the crack tip.

Next we compare the first order asymptotic solution of $y_{1}$ and $y_{2}$ in eq.(6) with the experimental data. There is only one undetermined parameter, i.e., the coefficient $A$. As 
will be shown later, we determine $A$ by fitting the asymptotic solution of the dominant deformation gradient component $F_{22}$ (see eq.(16)) to the experimental data and found $A=$ $=3.86 \mathrm{~mm}^{1 / 4}$. In Fig. $4 \mathrm{~b}$, we observe that the asymptotic solution of $y_{2}$ agrees well with the experimental result for $r$ up to $2.5 \mathrm{~mm}$. In contrast, large deviations between asymptotic solution and experimental results of $y_{1}$ are found unless $r$ is smaller than $\sim 0.7 \mathrm{~mm}$. Similarly, in terms of the angular dependence, Fig.4c shows that the asymptotic solution of $y_{1}$ starts to deviate from the experimental result when $r>0.75 \mathrm{~mm}$. For $y_{2}$, the asymptotic solution agrees well with experimental data for $|\theta| \sim 90^{\circ}$ and $r$ up to $2.5 \mathrm{~mm}$, while clear deviation is found in the region of $\mid \theta>\sim 90^{\circ}$ and $r>0.75 \mathrm{~mm}$. Based on these observations, we conclude that the region of validity for the asymptotic solution of $y_{1}$ is much smaller than that of $y_{2}$. In particular, the asymptotic solution of $y_{2}$ is valid in a region ahead of the crack tip $\left(\mid \theta<\sim 90^{\circ}\right)$ with a size on the order of a few millimeters.

(a)

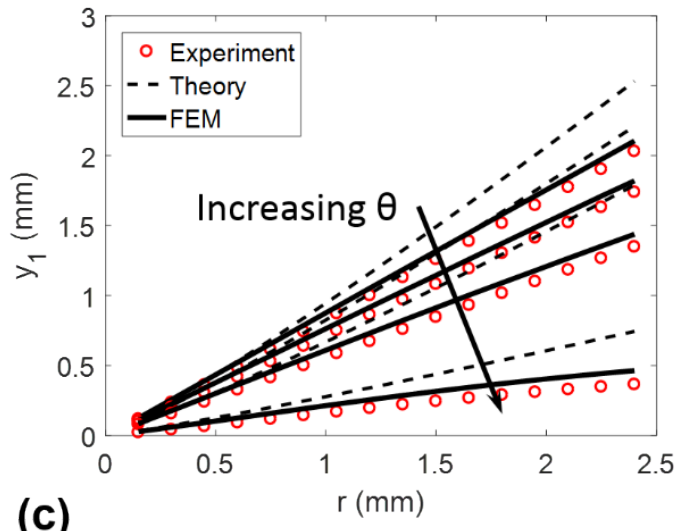

(c)

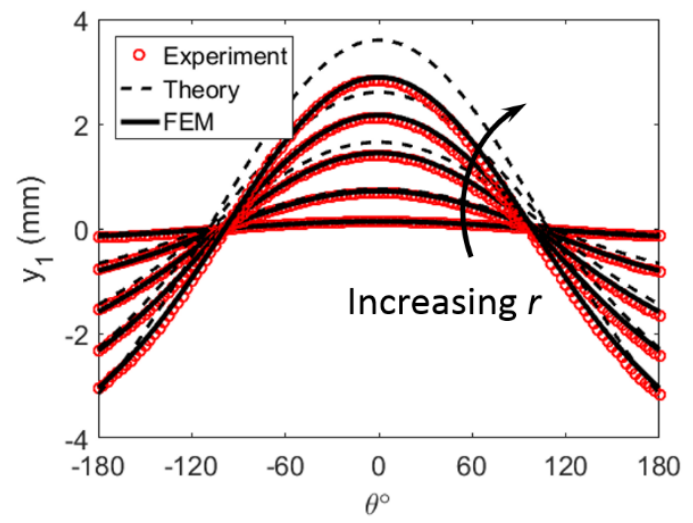

(b)
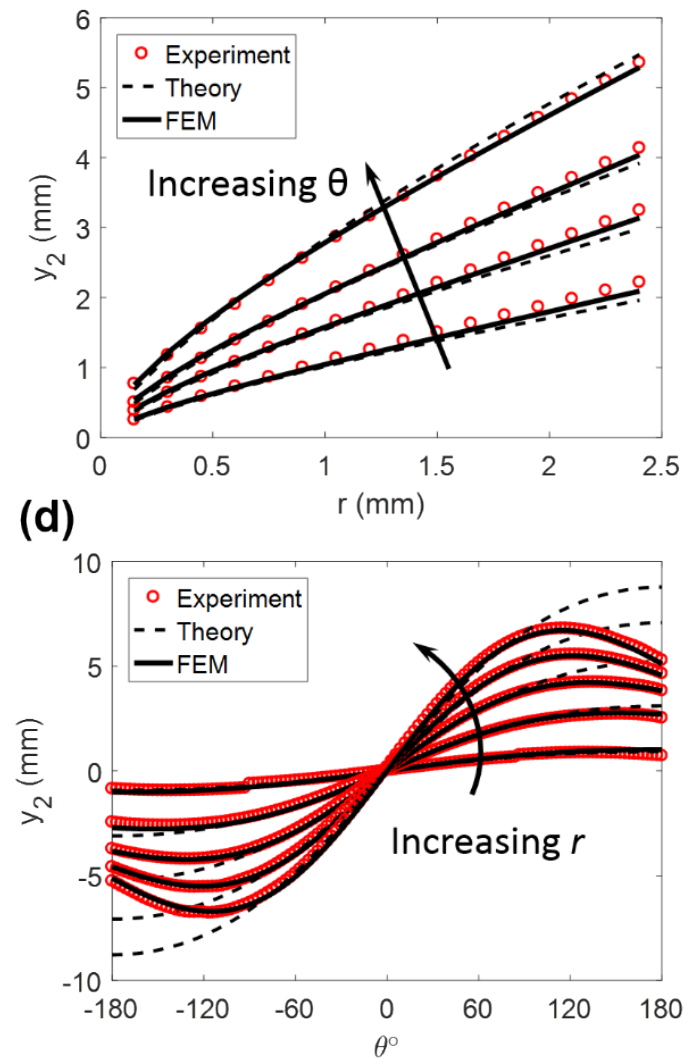
Figure 4 Comparing asymptotic solution, FEM and experimental results of the deformed coordinates $y_{1}$ and $y_{2}$. (a-b) $y_{1}$ and $y_{2}$ versus reference polar coordinate $r$. The arrows indicate the increasing $\theta\left(=30^{\circ}, 45^{\circ}, 60^{\circ}, 90^{\circ}\right)$. (c-d) $y_{1}$ and $y_{2}$ versus reference polar coordinate $\theta$. The arrows indicate increasing $r(=0.15,0.75,1.5,2.25,3.0 \mathrm{~mm})$. All results in (a)-(d) are for $\Delta / H_{0}=68.78 \%$.

(a)

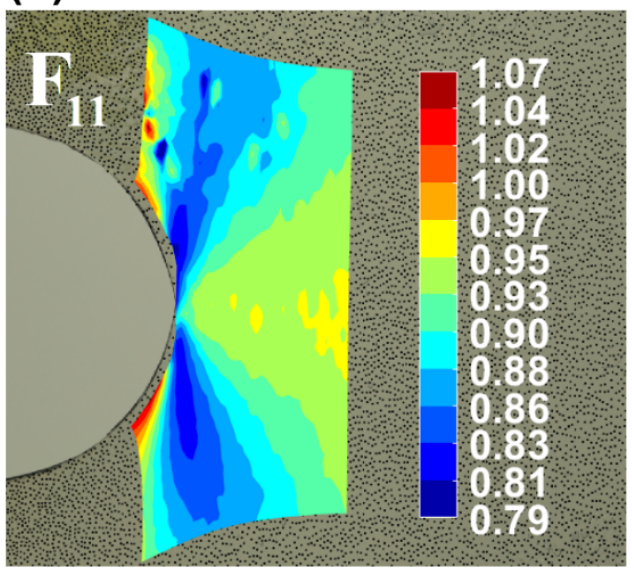

(c)

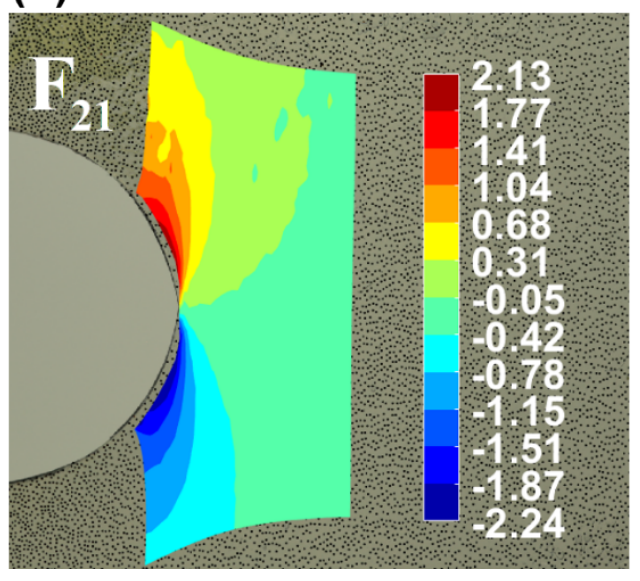

(b)

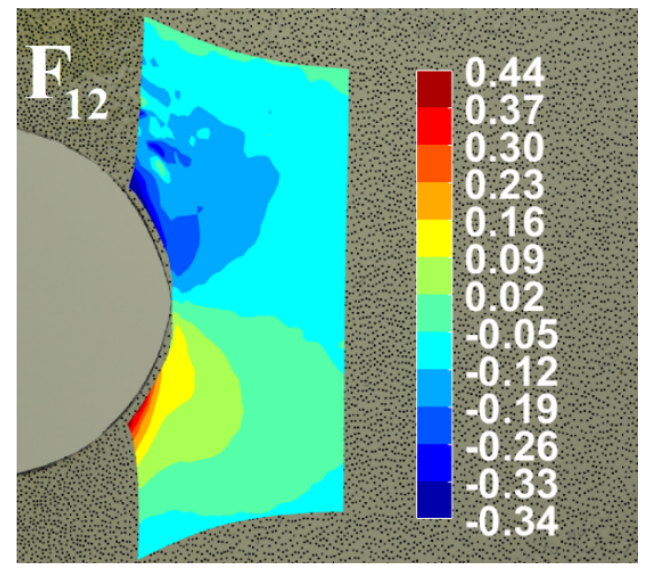

(d)

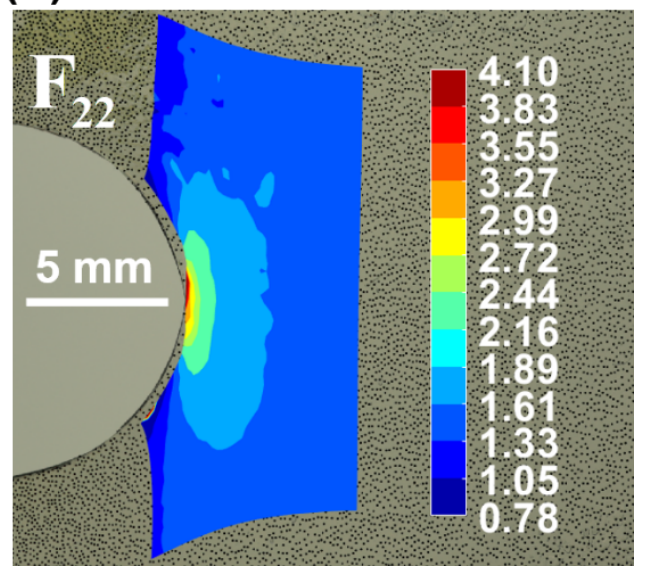

Figure 5 Experimentally measured multiaxial crack tip deformation field. Contour plots of the deformation gradient components: (a) $F_{11}$, (b) $F_{12}$, (c) $F_{21}$, (d) $F_{22}$. The subscripts 1 and 2 refer to the horizontal and vertical directions, respectively.

It is more important to consider the deformation gradient tensor $\mathbf{F}$, based on which strain and stress tensors can be calculated ${ }^{47,48}$. However, the measurement of $\mathbf{F}$ is more challenging than the displacement field. Since $\mathbf{F}$ involves the spatial gradients of $y_{1}$ and $y_{2}$, its accuracy is sensitive to the smoothness of the displacement field, i.e., even small uncertainties in the displacement data may lead to large errors in F. Using the MLS interpolation (see Section 2.4), we were able to generate a continuous displacement field 
from the discrete displacement data at tracer particles, and then evaluate $\mathbf{F}$ using gradients of the displacement field and eq.(12). Figure 5 shows how the four in-plane components of $\mathbf{F}$ are distributed around the deformed crack when $\Delta / H_{0}=68.78 \%$. In addition, a video illustrating evolution of these components along the loading history is provided in the supplementary material (see Appendix 3). We emphasize that the deformation gradient fields are directly measured from tracer particle displacements and thus does not require any knowledge regarding the mechanical property of the Ecoflex elastomer. Although the fields in Fig.5 appear to be smoother in the region below the midline of crack (i.e., $y_{2}<0$ or $\theta<0$ ) than that above the midline (i.e., $y_{2}>0$ or $\theta>0$ ), we can nevertheless see that $F_{11}$ and $F_{22}$ are approximately symmetric about the midline of the crack while $F_{12}$ and $F_{21}$ are antisymmetric about the midline, as expected from the Mode-I condition. In addition, Fig.5 shows that $F_{22}$ is the dominant component of $\mathbf{F}$, which is also expected from the Mode-I condition. The fact that $F_{22}$ can reach $\sim 4$ near the crack tip, implying a large nominal tensile strain of about $300 \%$. In the following we further examine the measured deformation gradient fields by quantitatively comparing them with the asymptotic solutions in eqs.(13)(16).

Figure 6a plots $F_{22}$ versus the reference radial coordinate $r$ directly ahead of the crack tip $\left(\theta=0^{\circ}\right)$. According to eq.(16), $F_{22}$ should scale with $r^{-1 / 4}$ as $r \rightarrow 0$ which is indeed observed in the experimental data. By fitting eq.(16) to this data, we find that the coefficient $A=3.86 \mathrm{~mm}^{1 / 4}$. Since $A$ is the only adjustable parameter, the entire deformation field predicted by the asymptotic solution is now determined. For example, dependence of $F_{22}$ on the reference angular coordinate $\theta$ with fixed $r$ is shown in Fig.6b. Moreover, the dependence of $F_{21}$ on $r$ and $\theta$ is plotted in Figs.6c-6d, respectively. In particular, since $F_{21}=0$ at $\theta=0^{\circ}$, in Fig. $6 \mathrm{c}$ we choose to plot $F_{21}$ along $\theta=-45^{\circ}$ since the measured field is smoother below the midline of crack. In addition, the angular dependence $F_{21}$ at 4 different radius $r$ is plotted separately in Fig.6d for better clarity. Overall the asymptotic solutions in eqs.(15) and (16) agree well with experimental data. In Fig.6a and 6c, deviation emerges when $r>\sim 1.5 \mathrm{~mm}$ which is attributed to the finite region of validity of the asymptotic solution. Even for $r<\sim 1.5 \mathrm{~mm}$, considerable deviation can be observed for $|\theta|$ close to $180^{\circ}$. Specifically, as marked in Fig.6d, the span of $\theta$ where eq.(15) agrees with experimental 
data (between the vertical dotted lines) decreases as $r$ increases. Since both $F_{21}$ and $F_{22}$ are related to the spatial gradients of $y_{2}$, the deviation for large $|\theta|$ can be traced back to Fig.4d where the asymptotic solution of $y_{2}$ deviates from the experimental data for large $|\theta|$.

(a)

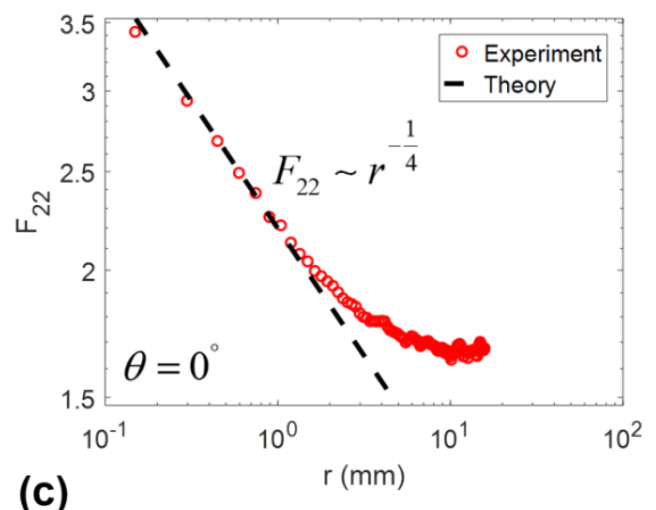

(c)

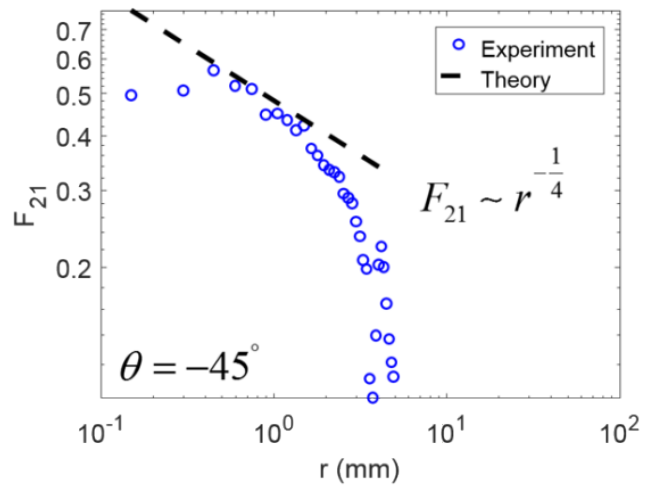

(b)
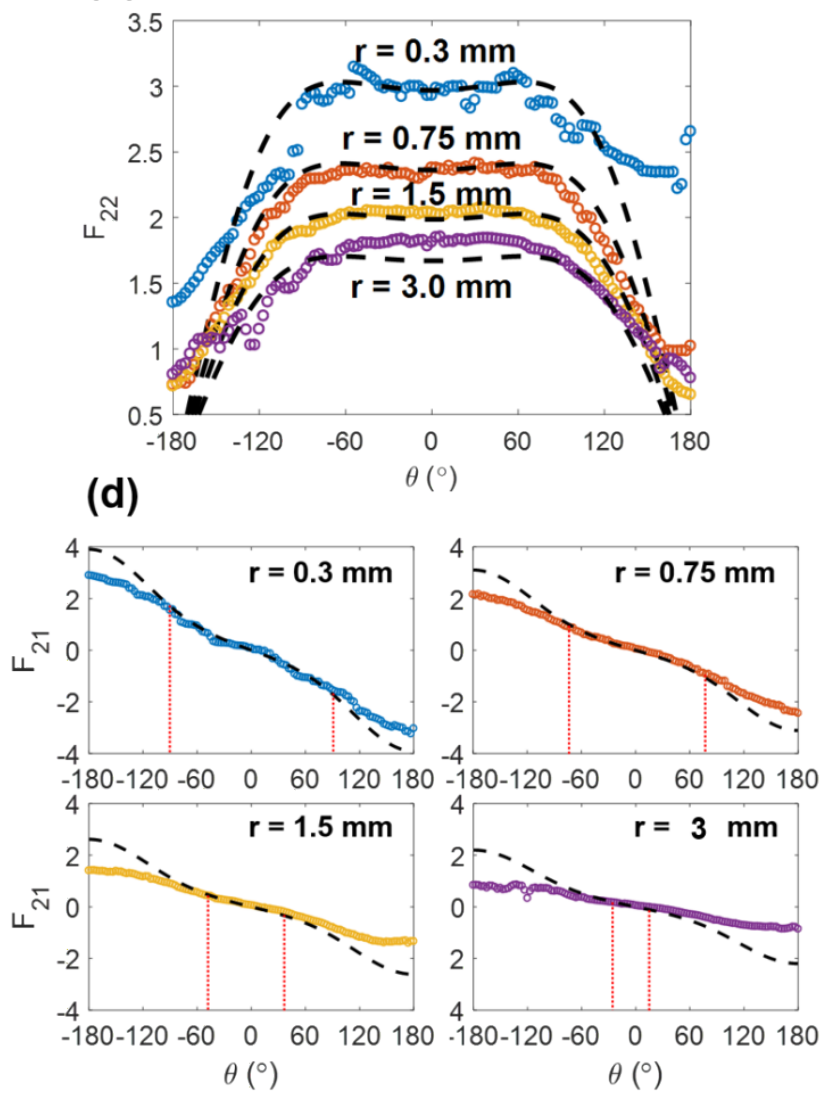

Figure 6 Comparing experimental data and asymptotic solution for $F_{22}$ and $F_{21}$. (a) Radial dependence of $F_{22}$ (log-log plot) directly ahead of the crack tip $\left(\theta=0^{\circ}\right)$. (b) Angular dependence of $F_{22}$ at four different radii $r$. (c) Radial dependence of $F_{21}$ (log-log plot) along $\theta=-45^{\circ}$. (d) Angular dependence of $F_{21}$ at four different radii $r$. In (a-d), the dashed lines are predicted by the asymptotic solution in eq.(15) or eq.(16). 
(a)

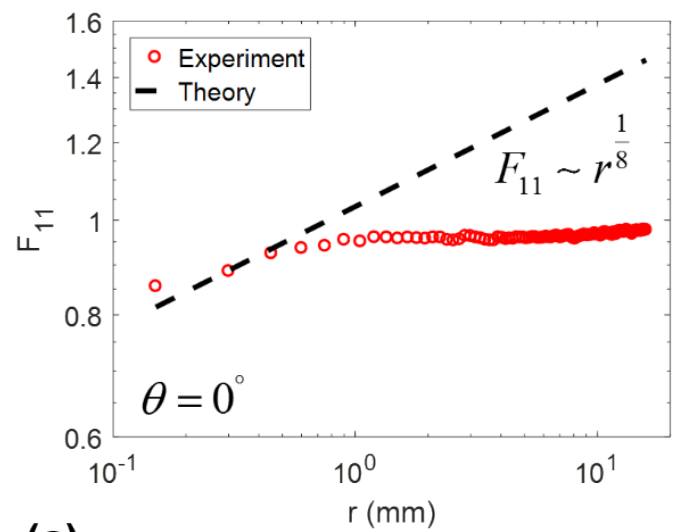

(c)

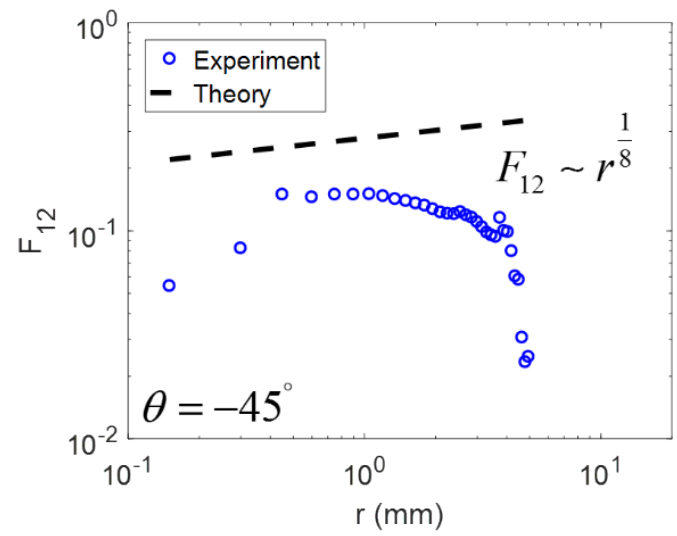

(b)

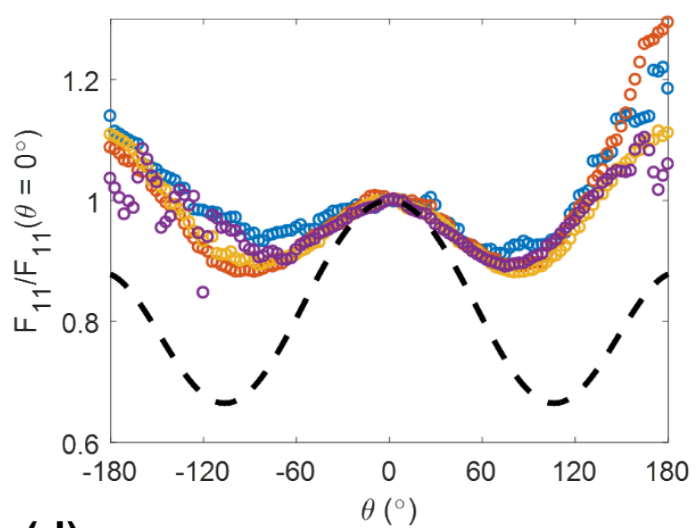

(d)

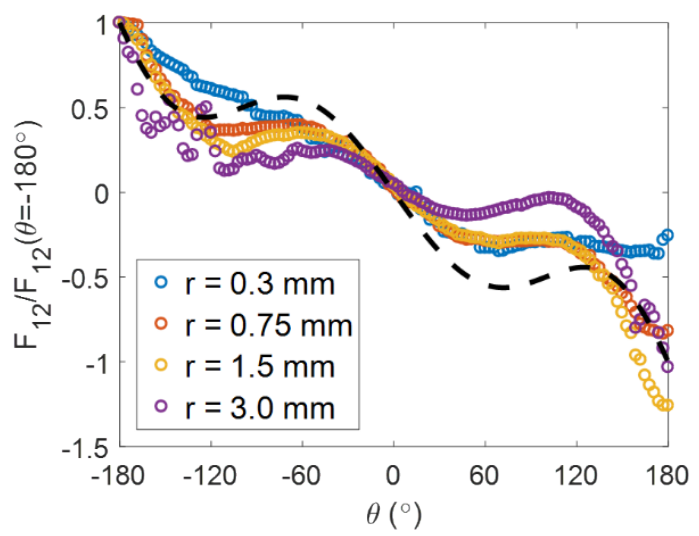

Figure 7 Comparing experimental data and asymptotic solution for $F_{11}$ and $F_{12}$. (a) Radial dependence of $F_{11}$ (log-log plot) directly ahead of the crack tip $\left(\theta=0^{\circ}\right)$. (b) Angular dependence of $F_{11}$ normalized by $F_{11}\left(r, \theta=0^{\circ}\right)$. (c) Radial dependence of $F_{12}$ (log-log plot) along $\theta=-45^{\circ}$. (d) Angular dependence of $F_{12}$ normalized by $F_{12}\left(r, \theta=-180^{\circ}\right)$. In (b) and (d), experimental data at 4 different radii (same color code) are plotted: $r=0.3 \mathrm{~mm}, 0.75 \mathrm{~mm}$, $1.5 \mathrm{~mm}$, and $3.0 \mathrm{~mm}$. The dashed lines are predicted by the asymptotic solution in eq.(13) or eq.(14).

As discussed earlier, the region of validity for the asymptotic solution of $y_{1}$ is smaller than that of $y_{2}$. Consequently, we expect the asymptotic solution of $F_{11}$ and $F_{12}$, which involve the spatial gradient of $y_{1}$, may deviate more from the experimental data than $F_{21}$ and $F_{22}$, which is confirmed by the comparison in Fig.7. In particular, Fig.7a plots the radial dependence of $F_{11}$ along $\theta=0^{\circ}$. The asymptotic solution in eq.(13) implies $F_{11}$ should scale with $r^{1 / 8}$, unlike $F_{22}$ which decays with $r$ as $r^{-1 / 4}$ (see Fig.6a). This scaling relation is observed in the experimental data shown in Fig.7a. Furthermore, since $A=3.86 \mathrm{~mm}^{1 / 4}$ and $g(\theta)$ is determined, there is no additional adjustable parameter in eq.(13). Yet quantitative 
agreement between eq.(13) and the experimental data can be found in Fig.7a when $r$ $<\sim 0.5 \mathrm{~mm}$. This region of validity is smaller than that of $F_{22}$ exhibited in Fig.6a. On the other hand, the scaling relation $F_{12} \sim r^{1 / 8}$, as predicted by eq.(14), is not found in the experimental data of Fig. $7 \mathrm{c}$ (along $\theta=-45^{\circ}$ since $F_{12}$ vanishes along $\theta=0^{\circ}$ ). To understand this discrepancy, we first assume the region of validity of eq.(14) is similar to that eq.(13), i.e., $r<\sim 0.5 \mathrm{~mm}$. However, within this small region, the magnitude of $F_{12}$ is much lower than $F_{11}$ and thus is more vulnerable to noises caused by experimental uncertainty in tracer particle displacement $(\sim 1$ pixel or $8 \mu \mathrm{m})$ and interpolation errors. Finally, the angular dependences of $F_{11}$ and $F_{12}$ at four different radii are shown in Figs. $7 \mathrm{~b}$ and $7 \mathrm{~d}$, respectively. Due to the small region of validity for the asymptotic solution, a quantitative agreement between asymptotic solution and experimental data is not expected. Interestingly, eqs.(13) and (14) can still capture the trend of the angular distribution of $F_{11}$ and $F_{12}$ with moderate relative error $(<\sim 25 \%)$.

In summary, we demonstrated that the asymptotic solution of crack tip field in a GNH hyperelastic solid (with $n=2$ ) can capture the deformation field measured in our experiments, especially for the dominant deformation gradient component $F_{22}$. This is the first time that the nonlinear asymptotic solution of crack tip deformation field based on hyperelasticity is verified experimentally.

\subsection{Effect of remote loading}

The previous section focuses on an exemplar snapshot (e.g. $\Delta / H_{0}=68.78 \%$ ). Since the experimental data covers the entire loading history, we are able to monitor evolution of the crack tip deformation field as the remote loading $\Delta / H_{0}$ increases. In Fig.8a-8b, we plot dependence of $F_{22}$ on the reference polar coordinates $r$ and $\theta$ for four different values of $\Delta / H_{0}$. In Fig.8a, we see that at small $\Delta / H_{0}=3.90 \%$ the experimental data deviates significantly from the scaling $F_{22} \sim r^{-1 / 4}$ predicted by the asymptotic solution in eq.(16). When the remote loading $\Delta / H_{0}$ increases, a region with $F_{22} \sim r^{-1 / 4}$ emerges and expands in size. Similar behavior is seen in Fig. $8 \mathrm{~b}$ where we compare the angular distribution of $F_{22}$ with eq.(16). To avoid the fitting parameter $A$, we normalize $F_{22}$ by $F_{22}\left(r, \theta=0^{\circ}\right)$. Again, the experimental data differs significantly from the dashed line given by eq.(16) at small 
$\Delta / H_{0}=3.90 \%$, but then converges to it as $\Delta / H_{0}$ increases. At small remote loading, the crack tip field is expected to follow the solutions provided in linear elastic fracture mechanics (LEFM). Therefore, we plot the radial dependence of the linear strain $\varepsilon_{22}=F_{22}$ -1 in Fig. $8 \mathrm{c}$ and find that $\varepsilon_{22}$ scales as $r^{-1 / 2}$ at small $\Delta / H_{0}$, i.e., the well-known square root singularity ${ }^{23}$, and then deviates it when $\Delta / H_{0}$ reaches $68.78 \%$. Interestingly, for moderate loadings (e.g., $\Delta / H_{0}=25 \%$ to $45 \%$ ), both the $\varepsilon_{22} \sim r^{-1 / 2}$ or $F_{22} \sim r^{-1 / 4}$ scaling exhibit a certain region of validity.

(a)

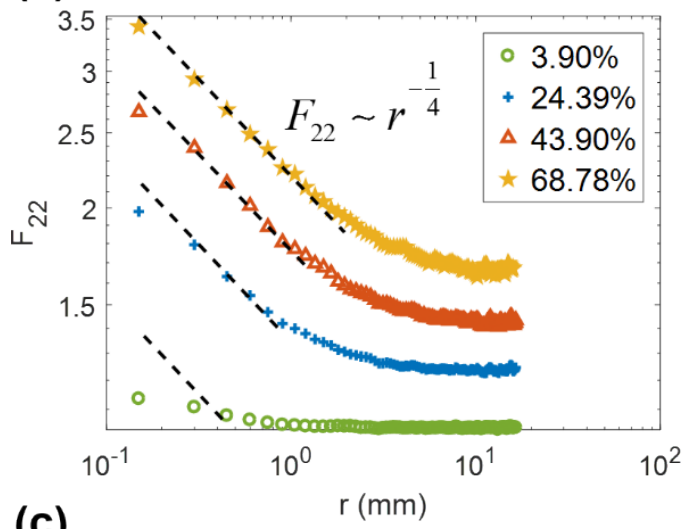

(c)

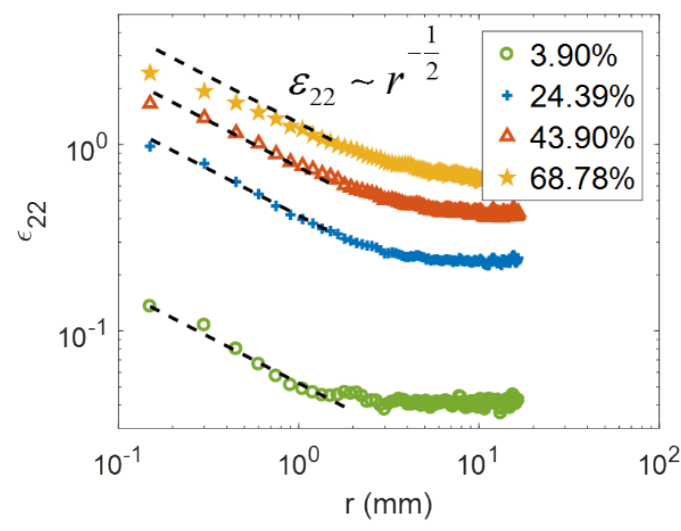

(b)
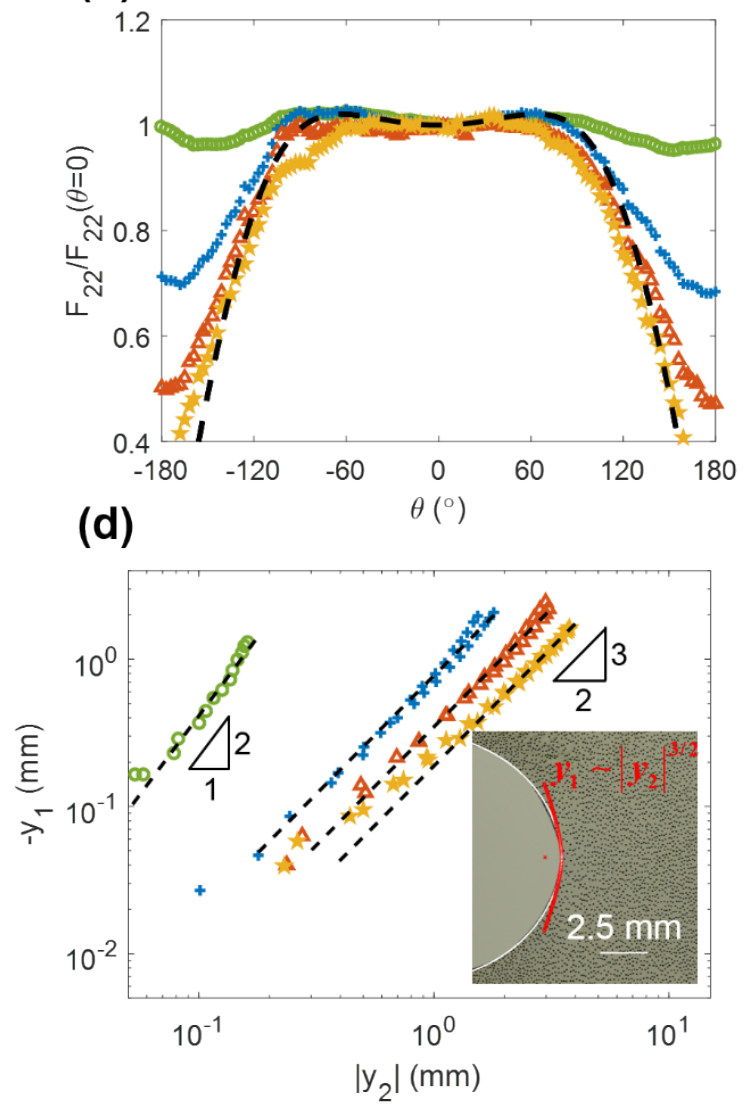

Figure 8 Crack tip deformation field transitions from LEFM solution to the nonlinear asymptotic solution when the remote loading increases. (a) Radial dependence of $F_{22}(\log$ $\log$ plot) directly ahead of the crack tip $\left(\theta=0^{\circ}\right)$. The dashed lines illustrate the scaling $F_{22}$ $\sim r^{-1 / 4}$. (b) Angular distribution of normalized $F_{22}$ at $r=1.5 \mathrm{~mm}$. The dashed line is given by the nonlinear asymptotic solution in eq.(16). (c) Radial dependence of linear strain $\varepsilon_{22}$ (log-log plot) directly ahead of the crack tip $\left(\theta=0^{\circ}\right)$. The dashed lines illustrate the scaling $\varepsilon_{22} \sim r^{-1 / 2}$ according to LEFM. (d) Scaling of the crack opening profile $-y_{1}$ versus $\left|y_{2}\right|$ (log$\log$ plot) as the remote loading increases. In (a-d), experimental data at 4 different remote loadings: $\Delta / H_{0}=3.9 \%, 24.39 \%, 43.9 \%$ and $68.78 \%$ are plotted with the same symbols illustrated in the legend of $(\mathbf{a}, \mathbf{c})$. The inset in (d) shows the experimental crack opening 
profile at $\Delta / H_{0}=68.78 \%$, FEM result (white line) and prediction of the asymptotic solution (red line).

The nonlinear effect is also reflected in the crack opening profile ${ }^{19}$. The LEFM solution predicts that the crack should deform into a parabola locally near the crack tip ${ }^{19,20}$. In contrast, by setting $\theta= \pm 180^{\circ}$ in eq.(6), we find that the nonlinear asymptotic solution predicts a different scaling for crack deformation: $-y_{1} \sim\left|y_{2}\right|^{3 / 2}$, which implies that the deformed crack is sharper than a parabola with scaling: $-y_{1} \sim\left|y_{2}\right|^{2}$. To verify this point, In Fig.8d we plot $-y_{1}$ and $\left|y_{2}\right|$ of the deformed crack surface using the values extracted from experimental images at the four remote loadings $\Delta / H_{0}$ shown in Fig.8a-b (same symbols). At small $\Delta / H_{0}(=3.90 \%),-y_{1}$ scales with $\left|y_{2}\right|^{2}$, indicating a parabolic crack profile. As $\Delta / H_{0}$ increases, nonlinear effect becomes dominant and the deformed crack profile transitions into the scaling $-y_{1} \sim\left|y_{2}\right|^{3 / 2}$ predicted by the nonlinear solution. The inset shows that at $\Delta / H_{0}=68.78 \%$ the local crack opening profile indeed follows the asymptotic solution in eq.(6) with $A=3.86 \mathrm{~mm}^{1 / 4}$.

The results in Fig.8 imply that as the remote loading increases, the region of validity for the LEFM solution becomes smaller and eventually disappears. In contrast, the nonlinear asymptotic solution exhibits an expanding region of validity as the remote loading increases. To demonstrate this point, we choose $F_{22}$ as the benchmark, and use our experimental data to define a region of validity within which the relative error between asymptotic solution and experimental data is below a threshold. Specifically, we first form a grid around the crack tip in the reference configuration using an increment of $0.15 \mathrm{~mm}$ in $r$ and $3^{\circ}$ in $\theta$, as shown in Fig.9a. Then the experimental value of $F_{22}$ at each grid point is compared with the asymptotic solution in eq.(16), and the grid points at which the relative error between the experimental data and the asymptotic solution is within a threshold of $4 \%$ are highlighted (see Fig.9a). These highlighted grid points approximately define the boundary of the region of validity. We assume the region of validity is a simply connected region. Therefore, even though close to the tip there are grid points where the relative error is higher than threshold (see Fig.9a), we still include them because such relative error is caused mainly by experiment uncertainties, e.g., imaging drift or slight loss of focus due to thinning of the sample near the crack tip. It should be noted that here we chose to focus 
on $F_{22}$ because it is the dominant deformation gradient component under Mode-I loading. Other deformation gradient components, e.g., $F_{11}$, may have different regions of validity. Using this method, we sketch the region of validity for the asymptotic solution of $F_{22}$ (see eq.(16)) at different remote loadings. Evolution of the region of validity shown in the reference and deformed configurations is given in Figs.9b and 9c, respectively. The region of validity expands as the remote loading increases $\left(\Delta / H_{0}\right.$ from $29.27 \%$ to $\left.68.78 \%\right)$ and then becomes steady when the crack starts to propagate $\left(\Delta / H_{0}=73.17 \%\right.$ and $\left.78.05 \%\right)$. This result can shed light on how nonlinear effects at the crack tip emerge and become dominant as the crack continues to deform.

(a)

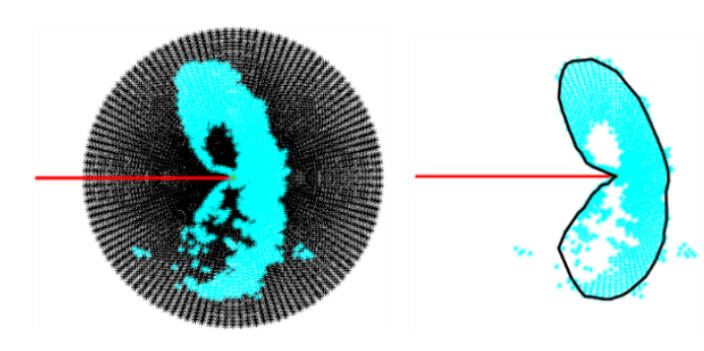

(c)
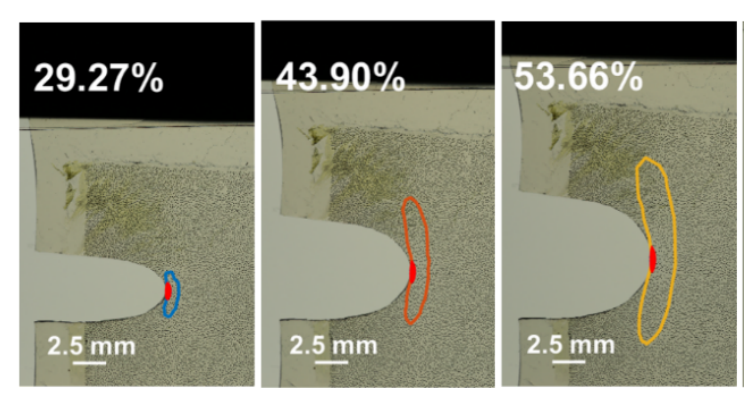

(b)
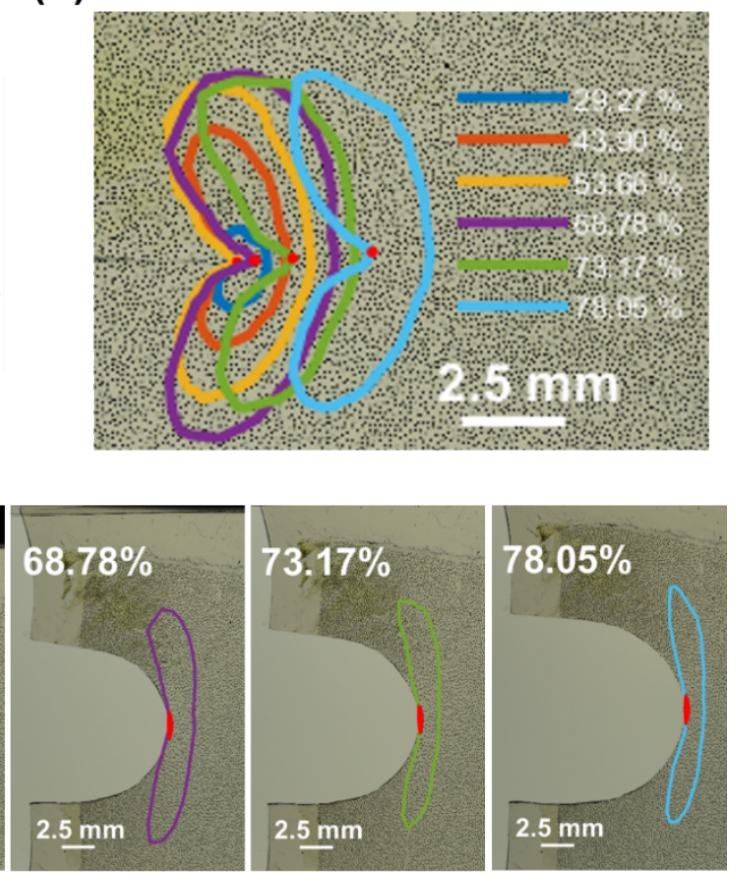

Figure 9 Region of validity for the nonlinear asymptotic solution expands as the remote loading increases. (a) Determining the region of validity for eq.(16). The red solid line represents the crack in the reference configuration, while grid points are shown as black stars. At the highlighted grid points (cyan stars), the experimental data of $F_{22}$ and asymptotic solution are within $4 \%$ relative error. (b-c) Region of validity for the nonlinear asymptotic solution of $F_{22}$ at different remote loadings $\Delta / H_{0}(29.27 \%$ to $78.05 \%)$. Part (b) and (c) show the reference and deformed configurations, respectively. A red dot with $r$ $<0.15 \mathrm{~mm}$ at the crack tip to illustrate a small region where the size is beyond the spatial resolution of our particle tracking method and thus the experimental data of $F_{22}$ may become inaccurate. 


\section{Energy release rate and fracture toughness}

The main purpose of fracture tests is to characterize a material's toughness $\Gamma$, defined as the work required to advance a crack by a unit area measured in the reference configuration. Following the Griffith's criterion ${ }^{23}$, crack propagation results from the competition between the energy release rate $G$, representing the energetic driving force supplied by external loading, and the toughness $\Gamma$. For the pure shear geometry (see Fig.2a), $G$ can be determined using:

$$
G=W\left(\lambda_{r}\right) H_{0}
$$

where $W$ is the strain energy density function, $H_{0}$ is the sample height and $\lambda_{r}=1+\Delta / H_{0}$ is the remote stretch ratio. The fracture toughness $\Gamma$ is determined by $\Gamma=G\left(\lambda_{r}=\lambda_{c}\right)$ where $\lambda_{c}$ is the critical remote stretch ratio at the onset of crack propagation. However, this approach relies on a global energy balance of the entire fracture sample, which is available for only a few test geometries ${ }^{12}$. For examples, the pure share geometry requires the sample width to be much larger than the height ${ }^{55}$, e.g., the width/height ratio in the literature ${ }^{15}$ is typically larger than 4. In addition, it is often necessary to measure the length of crack extension, e.g., for determining the velocity of crack propagation. However, this may not be straightforward if the crack tip is severely blunted where large deformation alone can displace the crack tip. These two issues can be addressed using the experimental data of crack tip deformation field in two aspects: i) local evaluation of $G$ through the J-integral ${ }^{57,58}$; ii) determination of crack extension length in the reference configuration.

\subsection{Evaluation of J-integral}

For the plane stress crack in our fracture experiments, the J-integral is ${ }^{57}$

$$
J=\int_{C}\left(W n_{1}-S_{\alpha \beta} n_{\beta} \frac{\partial u_{\alpha}}{\partial X_{1}}\right) d s,
$$

where $C$ is a contour in the reference configuration that encloses the crack tip, $W$ is the strain energy density function, $\mathbf{n}$ is the unit outward normal vector of $C, \mathbf{S}$ is the first PiolaKirchhoff stress tensor, $\mathbf{u}$ is the displacement vector and $s$ is the arc length of $C$. The 
subscripts $\alpha$ and $\beta$ range from 1 to 2 , and we have used the summation convention of summing over repeated indices. With the experimental data of crack tip deformation field, we can calculate $W$ using the GNH model in eq.(5) and components of $\mathbf{S}$ using

$$
S_{i j}=-p F_{j i}^{-1}+2 \frac{d W}{d I_{1}} F_{i j}, i, j=1,2,3
$$

The term $p$ is a Lagrange multiplier enforcing the incompressibility constraint and is not directly related to the deformation gradient $\mathbf{F}$. We take advantage of the plane stress condition, specifically $S_{33}=0$, to determine $p$ and obtain the following equation for $S_{\alpha \beta}$ :

$$
S_{\alpha \beta}=2 \frac{d W}{d I_{1}}\left(F_{\alpha \beta}-\lambda_{3}^{2} F_{\beta \alpha}^{-1}\right) \quad(\alpha, \beta=1,2),
$$

where $\lambda_{3}$ is the out-of-plane stretch ratio:

$$
\lambda_{3}=\frac{1}{F_{11} F_{22}-F_{12} F_{21}}
$$

With eq.(20) and the GNH model for $W$, we are able to evaluate the J-integral using experimental data of crack tip deformation field.

The J-integral should be path independent and equal to the energy release rate $G$ for elastic materials. We selected ten different integral paths (see Fig.10a) to examine the path independence of the $J$ evaluated from experimentally measured deformation field. As illustrated in Fig.10b-10c, the J-integral computed using the ten paths all agree with each other and are equal to the energy release rate $G$ evaluated using the global approach in eq.(17). Therefore, we have demonstrated the feasibility of using J-integral and the experimentally measured crack tip field to determine $G$. This method would allow us to access new loading conditions in fracture tests of soft elastic materials. 
(a)

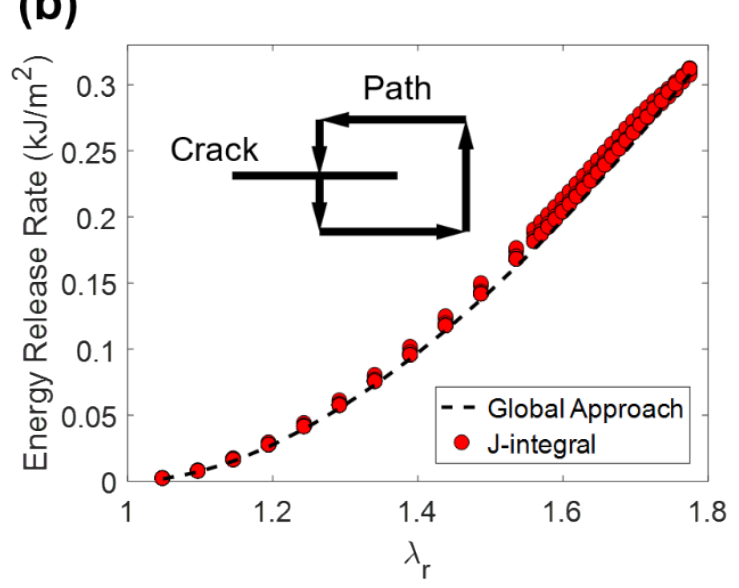

(c)

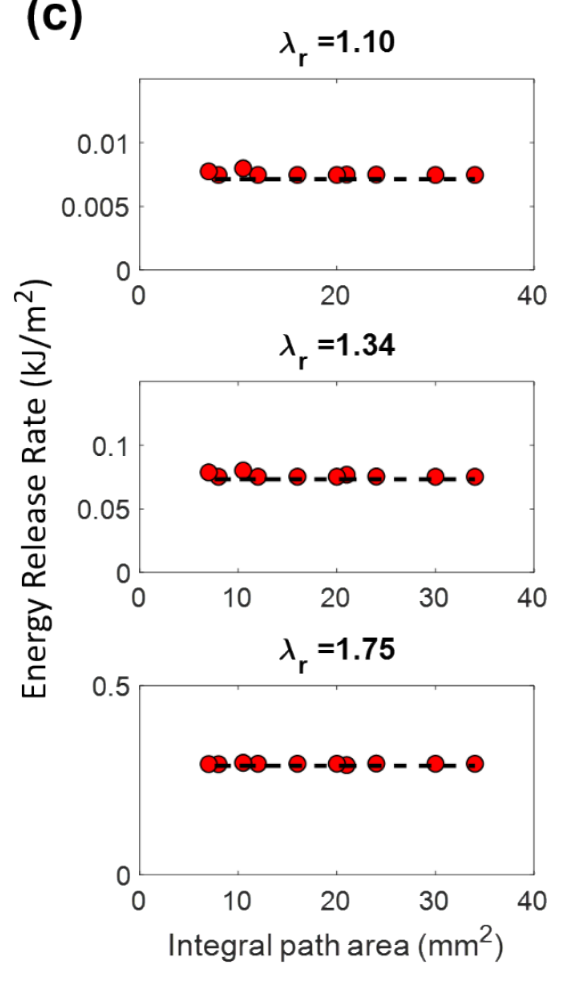

Figure 10 Path independence of the J-integral evaluated from experimental data. (a) Ten different integral paths around the crack tip in the reference configuration. (b) Energy release rate $G$ or J-integral versus the remote loading $\lambda_{r}=1+\Delta / H_{0}$. (c) The J-integral versus the area enclosed by different integral paths. In (b-c), the circular symbols represent Jintegrals evaluated from the experimental deformation data, while the dashed line represents the energy releaser rate $G$ evaluated using the global approach eq.(17).

\subsection{Measuring the crack extension length in reference configuration}

To measure the toughness $\Gamma$, we also need to determine when the crack starts to propagate by monitoring the position of the crack tip. However, in soft materials, the crack tip displacement may come from both deformation and crack propagation. Therefore, it could be difficult to precisely determine the onset of crack propagation. To decouple these two effects, we take advantage of the mapping between deformed and reference configurations established by our particle tracking method, and map the position of the current crack tip back to the reference configuration using an iterative numerical scheme described as follows. First, the crack tip $Q$ in current configuration is manually located in the image of the current frame (see Fig.11a). We make an initial guess of its corresponding 
position $P_{0}$ (see Fig. 11a) in the reference configuration, which is then fed into the moving least square (MLS) interpolation program to find the corresponding position $Q_{0}$ in the current configuration. Next we examine the distance $\left|\boldsymbol{\rho}_{Q Q}\right|$ between $Q_{0}$ and $Q$ and check if $\left|\boldsymbol{\rho}_{Q Q}\right|$ is below a small threshold $\eta$. If not, the reference position of the crack tip would be updated to a new point $P_{1}$ whose coordinates are equal to the coordinates of $P_{0}$ subtracted by $0.25 \rho_{Q Q^{\circ}}$. Following this scheme, we iteratively update the new reference position of the crack tip $P_{i}$ by subtracting the coordinates of $P_{i-1}$ by $0.25 \rho_{Q Q_{i-1}}$ until $\mid \rho_{Q Q_{i-1} \mid}$ is smaller than the threshold $\eta$.

Using this iterative scheme, we are able to monitor the position of crack tip in the reference configuration. The change in the reference crack tip location is solely due to crack propagation. In Fig.11b, we plot the energy release rate $G$ versus the crack extension length measured in the reference configuration. Initially the crack extension remains at 0 as $\Gamma$ increases until a critical value $\left(\Gamma_{0}=0.12 \mathrm{~kJ} / \mathrm{m}^{2}\right)$ is reached. After that, the crack tip accelerates as $G$ increases. The curve in Fig.11b is often referred to as the crack growth resistance curve ( $R$-curve) in the fracture mechanics literature ${ }^{23}$. If Ecoflex is exactly elastic, the $R$-curve should resemble a step function with a horizontal line leveled at $\Gamma_{0}$, because the crack would propagate unstably once $G$ exceeds $\Gamma_{0}$. The $R$-curve in Fig.11b implies that energy dissipation occurs near the crack tip, which causes an increase in the effective toughness $\Gamma$. This is consistent with the uniaxial tensile data showing that Ecoflex becomes inelastic at very large deformation (see Fig.A2). Such inelastic behavior leads to the formation of a dissipation zone around the crack tip, but this zone is much smaller than the detection limit of our particle tracking method, similar to the "small scale yielding" condition in $\mathrm{LEFM}^{23}$. Therefore, the Ecoflex elastomer used in our fracture experiments should be considered as "predominantly elastic".

To illustrate the limitation of using the deformed configuration to define crack extension, we plot in Fig.11c the same data of energy release rate $G$ versus the crack extension length measured using the crack tip location in the deformed configuration. The crack extension increases continuously with $G$, from which it is difficult to tell when exactly the crack propagation started. Furthermore, the definition of $G$, either using the global approach or the J-integral, are based on the crack surface area measured in the 
reference configuration. This also implies that it is more appropriate to associate $G$ with the reference crack extension length, instead of the deformed one.

(a)
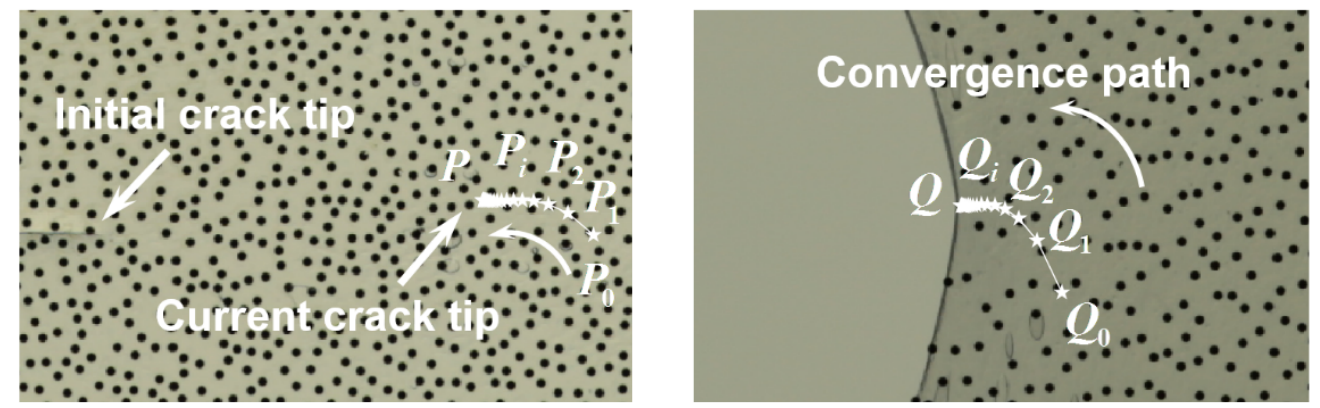

(b)

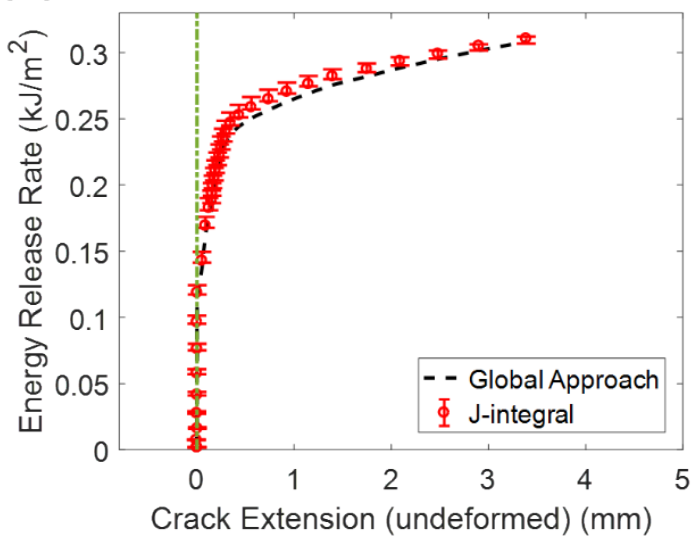

(c)

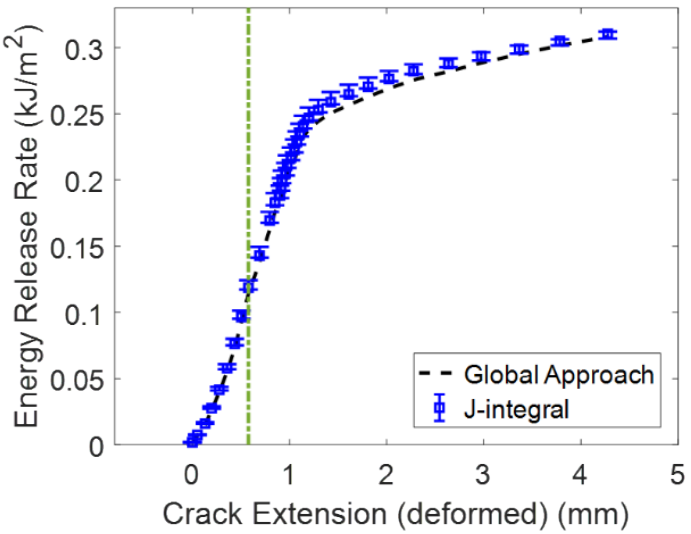

Figure 11 Onset of crack propagation unambiguously identified using the reference crack extension. (a) Iterative process of locating the crack tip in reference configuration. $P_{i}$ are the trial points in the reference configuration while $Q_{i}$ is the projection of $P_{i}$ in the deformed configuration. The converged crack tip position is ahead of initial crack tip due to crack propagation. (b-c) Energy release rate $G$ versus the crack extension length measured in (b) the reference configuration and (c) the deformed configuration. The symbols and error bars are average values and ranges of J-integrals using ten different paths, the dashed line is given by the global approach in eq.(17), and the vertical dashed line marks the onset of crack propagation.

\subsection{Rate dependence of fracture toughness}

The $R$-curve in Fig.11 implies that energy dissipation can occur near the crack tip. In this section, we study whether such crack tip dissipation is rate dependent. If the dissipation is rate-dependent, we expect the toughness $\Gamma$ to be positively correlated with crack propagation velocity $V$ can be established, as shown extensively for viscoelastic materials ${ }^{13,59,60}$. We further hypothesize that the $R$-curve would be sensitive to the loading 
history if the toughness $\Gamma$ is rate dependent. To test this hypothesis, we performed three pure shear fracture experiments with different loading histories shown in Fig.12. In all three experiments, the applied displacement $\Delta$ was first increased at a constant rate of $0.01 \mathrm{~mm} / \mathrm{s}$ followed by three different cases: i) Case I (sample height $H_{0}=19.5 \mathrm{~mm}$ ): $\Delta$ was held fixed when the peak force is reached; ii) Case II $\left(H_{0}=19.5 \mathrm{~mm}\right): \Delta$ was held fixed before the peak force is reached; iii) Case III $\left(H_{0}=20.0 \mathrm{~mm}\right)$ : $\Delta$ was continuously increased. The time histories of displacement and force in the three experiments are given in Fig.12.

(a)

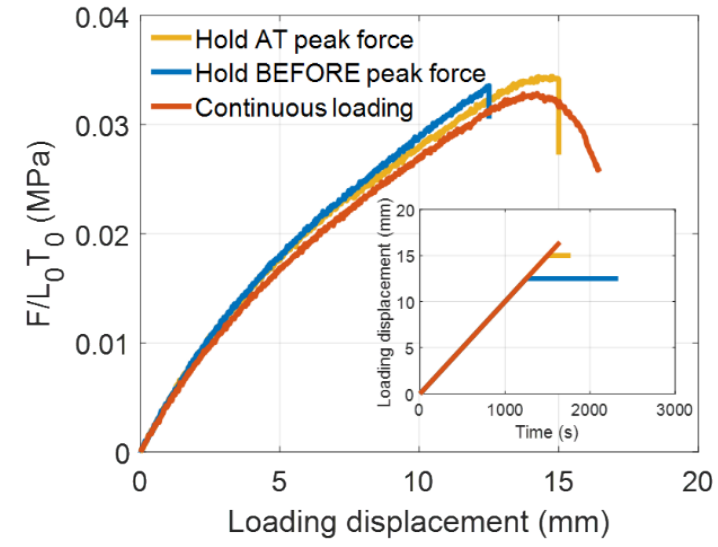

(b)

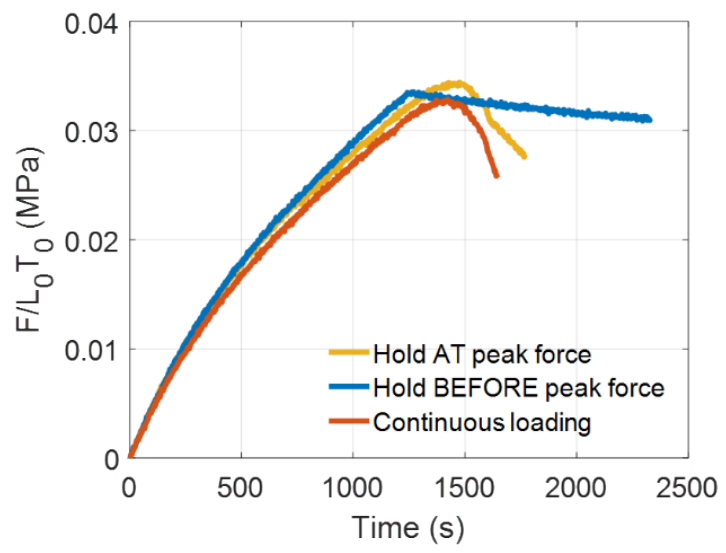

Figure 12 Fracture experiments with different loading histories. (a) Force versus displacement curves. The inset shows the time history of applied displacement $\Delta$. (b) Force versus time curves.

Using the history of the crack tip deformation established by the particle tracking method, we located the trajectories of the crack tip seen in the reference condition and plotted them in Fig.13a. Note that the $x_{1}-x_{2}$ axes are centered at the initial crack tip and are fixed in space. Therefore, the crack tip trajectory always starts at the origin of the $x_{1}-x_{2}$ plane and extends to the right as the crack propagates. Ideally the trajectory should be horizontal and aligned with the $x_{1}$ direction due to the Mode-I loading. However, we find that the trajectory may slightly deviate from the horizontal direction, likely due to blunting of the crack tip. We take the contour length of the trajectory as the crack extension length (reference configuration), and plot time history of the reference crack extension length in Fig.13b. 


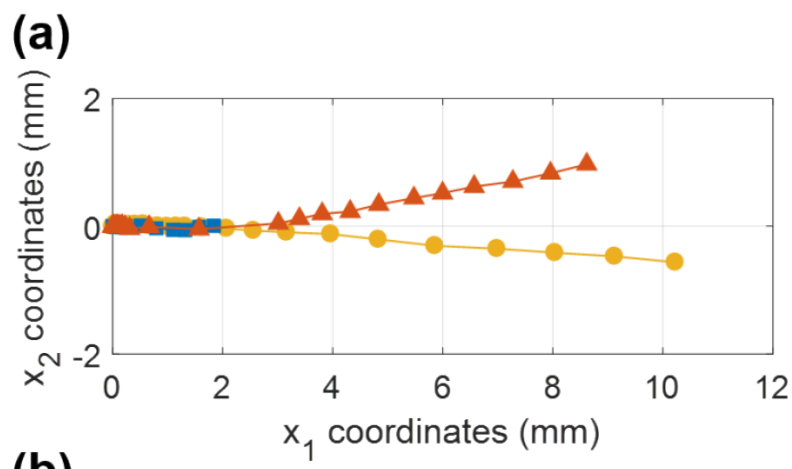

(b)

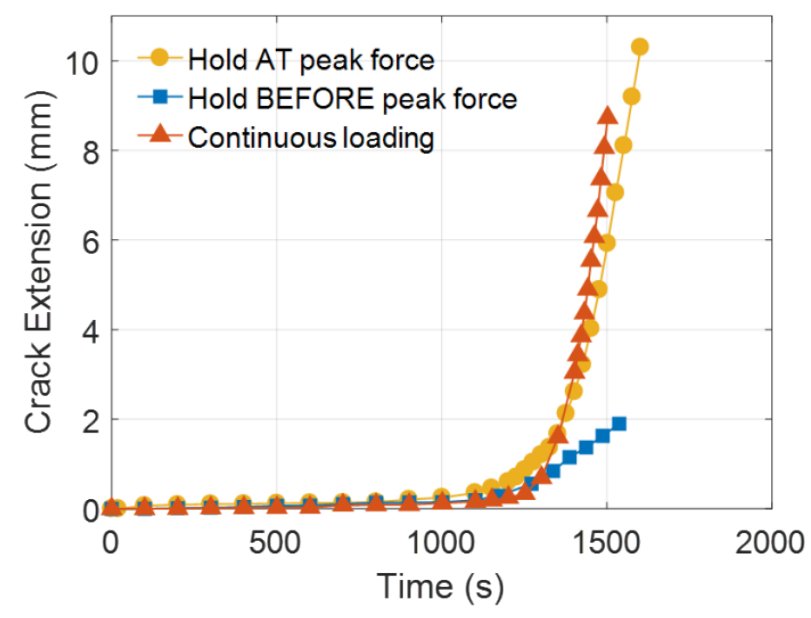

Figure 13 Tracking the reference crack extension length. (a) Trajectories of the crack tip measured in the reference configuration. (b) The reference crack extension length versus time.

From Fig.13, we observe that even in Case II ("hold before peak force"), the crack can still propagate but is much slower than the other two cases, which indicates that the toughness $\Gamma$ is not rate independent. To further illustrate the rate dependence of $\Gamma$, we plot the $R$-curves for the three cases in Fig.14a which was determined by first evaluating the energy release rate $G$ (using both the J-integral and global approach) as a function of time (see Fig.14b) and combing it with the crack extension length data in Fig.13b. Interestingly, results from the three experiments do not follow a single " $R$-curve" but are sensitive to the loading histories, which is another evidence for rate-dependent fracture toughness. Combing the data in Fig.13 and Fig14, we can extract the value of $\Gamma$ as well as the crack propagation velocity $V$ (measured in reference configuration) at each time increment. The results are plotted in Fig.15. Data from all three experiments with different loading histories collapse to a master curve showing a weak dependence of $\Gamma$ on the crack propagation 
velocity $V: \Gamma \sim V^{0.11}$. It is worth mentioning that the deviation of crack trajectory from horizontal direction seen in Fig.13a may result in slightly local mixed-mode conditions in the three experiments, which may contribute to the scattering of data in Fig.14. Nevertheless, we conclude that the crack tip dissipation process in the Ecoflex elastomer is rate-dependent, which further leads to a relation between the toughness $\Gamma$ and crack propagation velocity ${ }^{12}$.

(a)

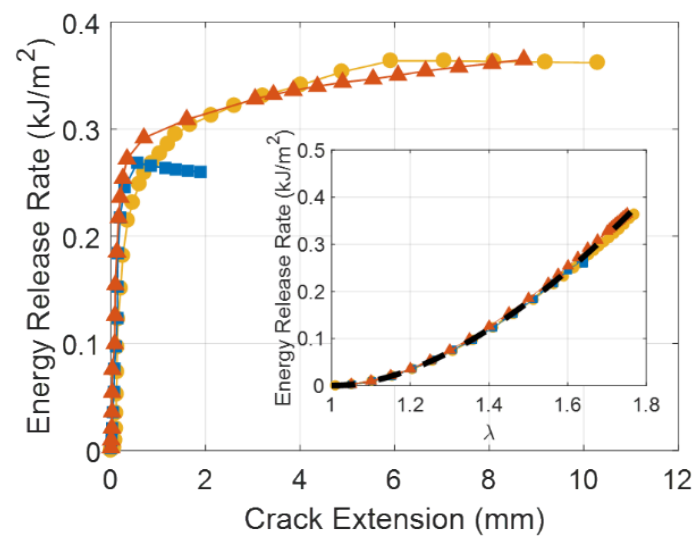

(b)

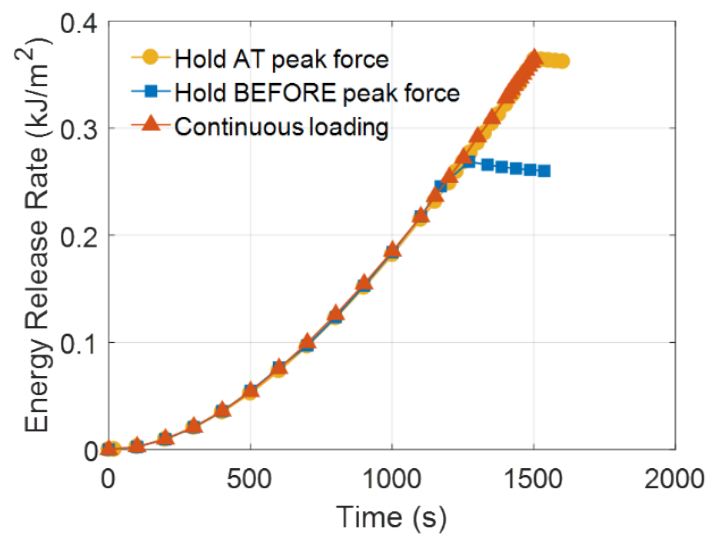

Figure 14 The $R$-curves under different loading histories. (a) Energy releaser rate $G$ calculated using the J-integral versus reference crack extension length for the three experiments. The inset shows that $G$ calculated using J-integral (symbols) agrees well with that from the global approach (dashed line). (b) Time history of $G$ for all three experiments.

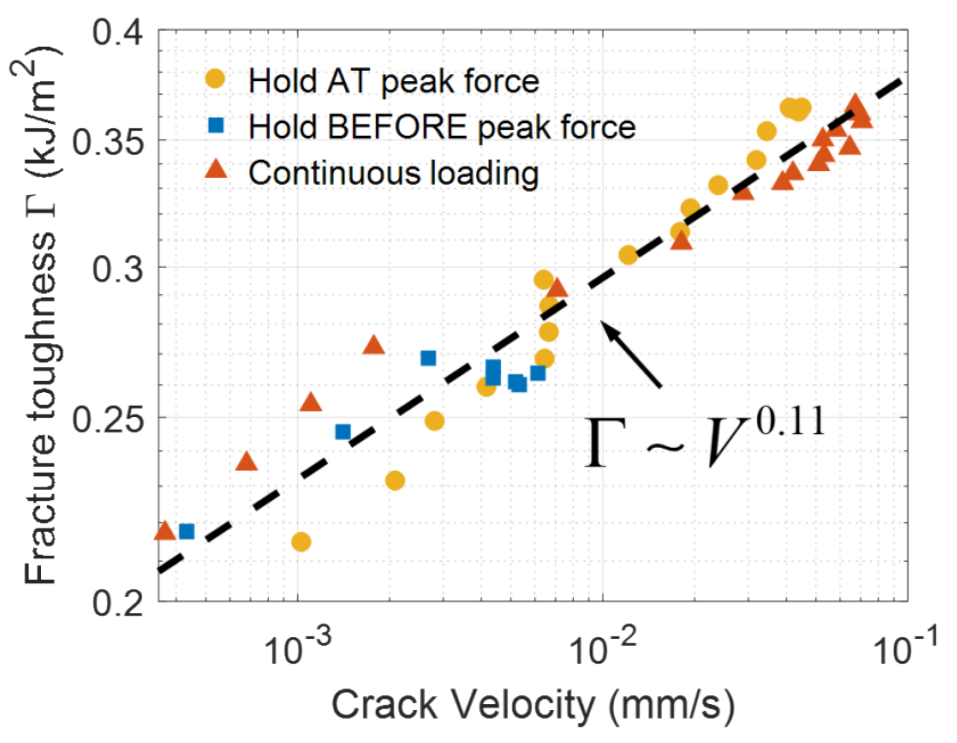


Figure 15 Dependence of the fracture toughness $\Gamma$ on crack propagation velocity $V$ measured in the reference configuration (log-log plot).

\section{Conclusions}

We demonstrated that the particle tracking method is capable of resolving the large and highly non-uniform crack tip deformation field in soft materials. Using a soft silicone elastomer, we experimentally observed, for the first time, the nonlinear crack tip field predicted by the asymptotic solutions for GNH hyperelastic solid. By monitoring the crack tip deformation field under increasing remote loading, we were able to capture how the region of validity for the nonlinear asymptotic solution evolves as the crack deforms and propagates.

For the measurement of fracture toughness, the experimental data of crack tip deformation field enables two new capabilities: i) local evaluation of the energy release rate $G$ through the J-integral; ii) determination of the true crack extension length defined in the reference configuration.

- The capability of measuring $G$ using the J-integral can be extended to any plane stress geometry, and thus can greatly expand the loading conditions and test geometries that can be used for soft material fracture. For example, by orientating the crack in the pure shear geometry (see Fig.2a) at an angle with the loading direction (e.g., 45), one may generate mixed Mode-I and Mode-II loadings near the crack tip even though the global loading is still tensile. This experiment could provide useful data for studying mixedmode fracture toughness in soft materials, which is not yet well understood.

- The capability of determining the reference crack extension length has two implications. First, for highly blunted cracks in soft materials, the onset of crack propagation, which must be determined for measuring the fracture toughness, can be ambiguous. Inspection of the crack tip position in the deformed configuration may be misleading due to the crack tip displacement caused by large deformation. Identifying the onset of crack propagation from the global force-displacement curve (e.g. see Fig.12) is not straightforward either. The experimentally measured history of crack tip deformation offers a much more precise way to detect crack extension, i.e., by monitoring the crack tip position in the reference configuration. Second, in current methods to measure the 
dependence of $\Gamma$ on crack velocity $V$, one often needs to wait until the crack propagation settles to a steady state with a constant velocity ${ }^{61-65}$. As a result, one fracture experiment can only yield one data point in the plot of $\Gamma$ versus $V$, which can be very time consuming. Here we demonstrate that, with an accurate measurement of crack extension, it is possible to obtain much more $\Gamma-V$ data points from a single experiment (see Fig.15). The could greatly reduce the experimental cost for characterizing the rate dependence of fracture toughness.

The particle tracking method can serve as a useful experimental tool to probe the mechanics of soft materials under extreme deformation. By directly mapping the crack tip deformation field, this method can lead to new advancements in soft material fracture. For example, for soft materials with strong dissipation and hence high toughness, by measuring the time history of crack tip deformation field and combining the data with a bulk constitutive model, it is possible to determine the amount of energy dissipation associated with crack propagation. The crack tip deformation data, when interpreted with FEM analysis, may offer new insights towards selecting appropriate local failure criteria at the crack tip to simulate fracture. Moreover, the particle tracking method can be extended to map the full three-dimensional (3D) deformation field within the volume of transparent hydrogels or elastomers ${ }^{47,67}$. This can potentially result in unprecedented data to reveal the mechanical structure of 3D cracks under complex loading conditions (e.g., mix-mode loadings).

\section{Appendix 1: Preparation of Ecoflex samples and tracer particles}

The Ecoflex samples were prepared in two steps (see Fig.A1): 1) fabricate a distribution of tracer particles with photo-lithography on a glass slide; 2) transfer the tracer particles onto an Ecoflex 0030 (Smooth-On Inc.) sheet. Briefly, a photo mask with random tracer particle pattern (particle diameter $\sim 0.1 \mathrm{~mm}$ ) was designed and ordered from $\mathrm{CAD} /$ Art Services, Inc. A clean glass slide was prepared, and spin coated with $1 \mu \mathrm{m}$ thick PDMS (3000rpm for 60s), which was then cured. After oxygen plasma treatment (RIE Jupiter) of the PDMS layer, polyimide (Sigma Aldrich) was then spin coated on top of it (4000rpm for $60 \mathrm{~s}$ ), and cured at $250^{\circ} \mathrm{C}$ for 1 hour. After cooling down, a 100nm copper 
layer was deposited on top of the polyimide film in a thermal evaporator. The copper layer was introduced to enhance the contrast of the tracer particles and thus improve the imaging quality. After that, a layer of AZ4210 photoresist was spin coated on top of the copper layer to transfer the tracer particle pattern from the photo mask (2000rpm for $60 \mathrm{~s} ; 120^{\circ} \mathrm{C}$ for 3 mins on hot plate and 60s exposure to $360 \mathrm{~nm}$ UV light). Copper etchant CE-100 was used as received to etch the pattern out of the copper layer, and the patterned sample was placed in RIE to remove the exposed polyimide. Next the pattern was transferred to a water soluble tape (Bertech Water Soluble Tape) at $100^{\circ} \mathrm{C}$. An Ecoflex sheet of $1 \mathrm{~mm}$ thickness was prepared and spin coated with another layer of liquid Ecoflex of $1 \mu \mathrm{m}$ thickness to improve bonding with the tracer particles. The water soluble tape was adhered to the Ecoflex sample with the particle side facing down, and then the liquid Ecoflex layer was cured at $80^{\circ} \mathrm{C}$ for 30 minutes. The water soluble tape was washed away with DI water, leaving the tracer particles on top of the Ecoflex sheet. Finally, the Ecoflex sheet with tracer particles was cut into the pure shear geometry, and a crack was introduced by a scalpel for fracture test.

a

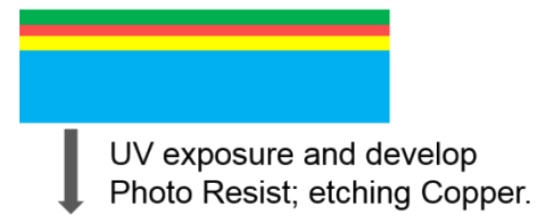

b

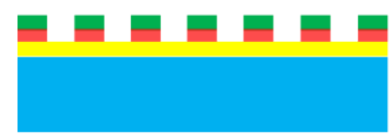

Remove Photo Resist and applied Water soluble tape.

C

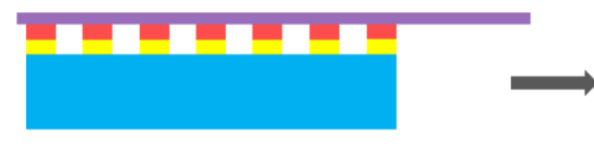

- AZ 4210 Photo resist

- Copper $100 \mathrm{~nm}$

- Glass sheet (PDMS coated) f $-a b-a$

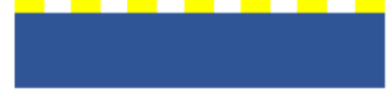

Dissolve Water soluble tape using DI water.

e

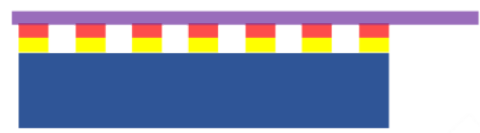

Transfer patterns on Ecoflex substrate using liquid Ecoflex as adhesive.

d Quickly peel off, and transfer patterns to the tape.

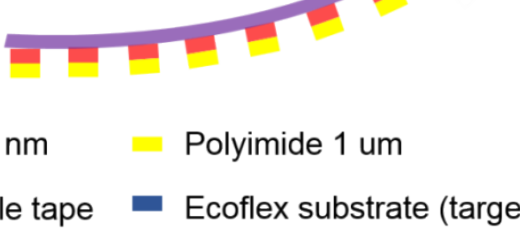

Figure A1 Schematic illustrating the process of depositing ultra-thin copper/polyimide islands as tracer particles on the surface of Ecoflex elastomer samples.

\section{Appendix 2: Cyclic tensile data for Ecoflex elastomer}


Cyclic tensile test data are shown in Fig.A2 where three samples experienced loading-unloading cycles at different strain rates. It can be seen that if the max stretch ratio experienced by the sample, $\lambda_{\max }$, is below 3 , the hysteresis loop is negligibly small and the elastomer can be considered to be nonlinearly elastic. However, if $\lambda_{\max }$ exceeds 3 , significant loading-unloading hysteresis can be observed, indicating inelastic behaviors in the material. Similar behavior has been observed in the literature ${ }^{66}$.

Although significant hysteresis can occur when $\lambda_{\max }$ exceeds 3 , we can still model the Ecoflex elastomer as a GNH hyperelastic solid in our fracture tests for two reasons. First, our experimental data suggest that during the entire course of crack propagation the maximum principal stretch may exceed 3 only in a small region very close to the crack tip (size $\sim 0.3 \mathrm{~mm}$ measured in the reference configuration; see Fig.5d). Outside this small region, the elastomer is practically elastic. As a result, the J-integrals are not affected by the inelastic behavior of the Ecoflex elastomer at large stretch since they are based on paths outside than the inelastic region. Second, even for $\lambda_{\max }$ larger than 3 , the loading branch is still well captured by the GNH model in eq.(5) (see Fig.A2), and therefore the elastomer can be treated as a GNH solid for interpreting the crack tip deformation field before unloading occurs.

a

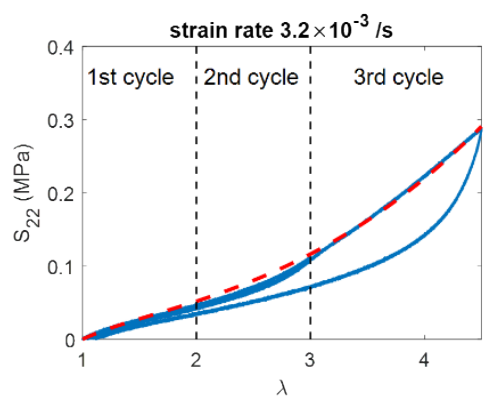

b

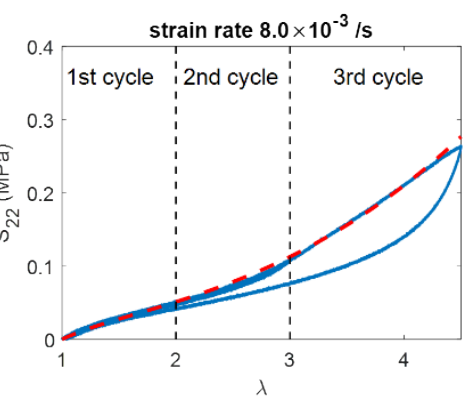

C

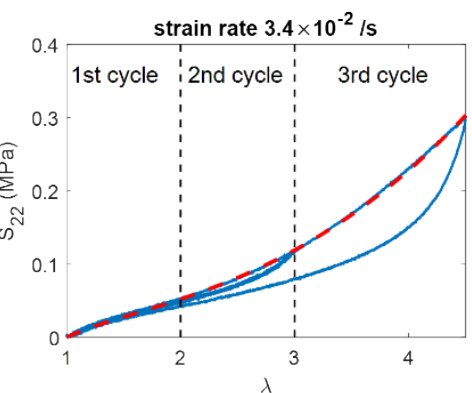

Figure A2 Cyclic tensile test data for three samples prepared from the same batch with different strain rates. The red dash lines are fits using the GNH model with $n=2$. The shear modulus $\mu$ and hardening parameter $b$ are: (a) $\mu=25.2 \mathrm{kPa}$ and $b=0.18$, (b) $\mu=25.1 \mathrm{kPa}$ and $b=0.167$, (c) $\mu=25.0 \mathrm{kPa}$ and $b=0.195$.

\section{Appendix 3: Supplementary video}


This video shows the time evolution of all deformation gradient components $F_{11}$, $F_{12}, F_{21}$ and $F_{22}$ with a remote loading rate of $0.01 \mathrm{~mm} / \mathrm{s}$. The video is accelerated and is 50 time faster than the real time.

\section{Acknowledgement}

This work was supported by the National Science Foundation under grant number CMMI-1636203. R.L. also acknowledges support from a CAREER award from the National Science Foundation (CMMI-1752449) and the 3M non-Tenured Faculty award. The authors are thankful to Xinzeng Feng for his guidance on the particle tracking algorithm.

\section{References}

1. Wojtecki, R. J., Meador, M. A. \& Rowan, S. J. Using the dynamic bond to access macroscopically responsive structurally dynamic polymers. Nat. Mater. 10, 14-27 (2011).

2. Calvert, P. Hydrogels for Soft Machines. Adv. Mater. 21, 743-756 (2009).

3. Drury, J. L. \& Mooney, D. J. Hydrogels for tissue engineering: Scaffold design variables and applications. Biomaterials 24, 4337-4351 (2003).

4. Morin, S. A. et al. Camouflage and Display for Soft Machines. Science (80-. ). 337, 828-832 (2012).

5. Park, S.-J. et al. Phototactic guidance of a tissue-engineered soft-robotic ray. Science 353, 158-62 (2016).

6. Yuk, H. et al. Hydraulic hydrogel actuators and robots optically and sonically camouflaged in water. Nat. Commun. 8, 14230 (2017).

7. Yasuda, K. et al. Biomechanical properties of high-toughness double network hydrogels. Biomaterials 26, 4468-4475 (2005).

8. Rogers, J. A., Someya, T. \& Huang, Y. Materials and mechanics for stretchable 
electronics. Science (80-. ). 327, 1603-7 (2010).

9. Keplinger, C. et al. Stretchable, Transparent, Ionic Conductors. Science (80-. ). 341, 984-987 (2013).

10. Lin, S. et al. Stretchable Hydrogel Electronics and Devices. Adv. Mater. 28, 44974505 (2016).

11. Chen, C., Wang, Z. \& Suo, Z. Flaw sensitivity of highly stretchable materials. Extrem. Mech. Lett. 10, 50-57 (2017).

12. Creton, C. \& Ciccotti, M. Fracture and adhesion of soft materials: a review. Reports Prog. Phys. 79, (2016).

13. Gent, A. N. Adhesion and Strength of Viscoelastic Solids. Is There a Relationship between Adhesion and Bulk Properties? †. Langmuir 12, 4492-4496 (1996).

14. Gong, J. P., Katsuyama, Y., Kurokawa, T. \& Osada, Y. Double-network hydrogels with extremely high mechanical strength. Adv. Mater. 15, 1155-1158 (2003).

15. Sun, J.-Y. et al. Highly stretchable and tough hydrogels. Nature 489, 133-136 (2012).

16. Sun, T. L. et al. Physical hydrogels composed of polyampholytes demonstrate high toughness and viscoelasticity. Nat Mater 12, 932-937 (2013).

17. Ducrot, E., Chen, Y., Bulters, M., Sijbesma, R. P. \& Creton, C. Toughening Elastomers with Sacrificial Bonds and Watching Them Break. Science (80-. ). 344, 186-189 (2014).

18. Zhao, X. Multi-scale multi-mechanism design of tough hydrogels: building dissipation into stretchy networks. Soft Matter 10, 672-87 (2014).

19. Livne, A., Bouchbinder, E., Svetlizky, I. \& Fineberg, J. The Near-Tip Fields of Fast Cracks. Science (80-. ). 327, 1359-1363 (2010).

20. Long, R. \& Hui, C. Y. Crack tip fields in soft elastic solids subjected to large quasi-static deformation - A review. Extrem. Mech. Lett. 4, 131-155 (2015).

21. Zhang, T., Lin, S., Yuk, H. \& Zhao, X. Predicting fracture energies and crack-tip 
fields of soft tough materials. Extrem. Mech. Lett. 4, 1-8 (2015).

22. Qi, Y., Caillard, J. \& Long, R. Fracture toughness of soft materials with rateindependent hysteresis. J. Mech. Phys. Solids 118, 341-364 (2018).

23. Zehnder, A. T. Energy Flows in Elastic Fracture. in Fracture Mechanics 33-54 (2012). doi:10.1007/978-94-007-2595-9_3

24. Wang, X. \& Hong, W. Pseudo-elasticity of a double network gel. Soft Matter 7, $8576(2011)$.

25. Zhao, X. A theory for large deformation and damage of interpenetrating polymer networks. J. Mech. Phys. Solids 60, 319-332 (2012).

26. Long, R. \& Hui, C.-Y. Fracture toughness of hydrogels: measurement and interpretation. Soft Matter 12, 8069-8086 (2016).

27. Tracy, J., Waas, A. \& Daly, S. Experimental assessment of toughness in ceramic matrix composites using the J-integral with digital image correlation part II: application to ceramic matrix composites. J. Mater. Sci. 50, 4659-4671 (2015).

28. Tracy, J., Waas, A. \& Daly, S. Experimental assessment of toughness in ceramic matrix composites using the J-integral with digital image correlation part I: methodology and validation. J. Mater. Sci. 50, 4646-4658 (2015).

29. Creton, C. \& Ciccotti, M. Fracture and adhesion of soft materials: A review. Reports on Progress in Physics 79, 046601 (2016).

30. Seitz, M. E. et al. Fracture and large strain behavior of self-assembled triblock copolymer gels. Soft Matter 5, 447-456 (2009).

31. Le Cam, J.-B. A Review of the Challenges and Limitations of Full-Field Measurements Applied to Large Heterogeneous Deformations of Rubbers. Strain 48, 174-188 (2012).

32. Sutton, M. A., Orteu, J.-J. \& Schreier, H. W. Digital Image Correlation (DIC). in Image Correlation for Shape, Motion and Deformation Measurements 1-37 (Springer US, 2009). doi:10.1007/978-0-387-78747-3_5

33. Vacher, P., Dumoulin, S., Morestin, F. \& Mguil-Touchal, S. Bidimensional strain 
measurement using digital images. Proc. Inst. Mech. Eng. Part C J. Mech. Eng. Sci. 213, 811-817 (1999).

34. Pan, B., Qian, K. M., Xie, H. M. \& Asundi, A. Two-dimensional digital image correlation for in-plane displacement and strain measurement: a review. Meas. Sci. Technol. 20, 17 (2009).

35. Franck, C., Hong, S., Maskarinec, S. A., Tirrell, D. A. \& Ravichandran, G. Threedimensional Full-field Measurements of Large Deformations in Soft Materials Using Confocal Microscopy and Digital Volume Correlation. Exp. Mech. 47, 427438 (2007).

36. Bar-Kochba, E., Toyjanova, J., Andrews, E., Kim, K. S. \& Franck, C. A Fast Iterative Digital Volume Correlation Algorithm for Large Deformations. Exp. Mech. 55, 261-274 (2015).

37. Landauer, A. K., Patel, M., Henann, D. L. \& Franck, C. A q-Factor-Based Digital Image Correlation Algorithm (qDIC) for Resolving Finite Deformations with Degenerate Speckle Patterns. Exp. Mech. 58, 815-830 (2018).

38. Witz, J.-F. et al. Digital Image Correlation for Large Strain. in 163-167 (Springer, Cham, 2017). doi:10.1007/978-3-319-51439-0_39

39. Pan, B., Dafang, W. \& Yong, X. Incremental calculation for large deformation measurement using reliability-guided digital image correlation. Opt. Lasers Eng. 50, 586-592 (2012).

40. Berfield, T. A. et al. Fluorescent Image Correlation for Nanoscale Deformation Measurements. Small 2, 631-635 (2006).

41. Berfield, T. A. et al. Micro- and Nanoscale Deformation Measurement of Surface and Internal Planes via Digital Image Correlation. Exp. Mech. 47, 51-62 (2007).

42. Han, Y., Rogalsky, A. D., Zhao, B. \& Kwon, H. J. The application of digital image techniques to determine the large stress-strain behaviors of soft materials. Polym. Eng. Sci. 52, 826-834 (2012).

43. Lin, S., Zhou, Y. \& Zhao, X. Designing extremely resilient and tough hydrogels 
via delayed dissipation. Extrem. Mech. Lett. 1, 70-75 (2014).

44. Krehbiel, J. D., Lambros, J., Viator, J. A. \& Sottos, N. R. Digital Image Correlation for Improved Detection of Basal Cell Carcinoma. Exp. Mech. 50, 813824 (2010).

45. Grytten, F., Daiyan, H., Polanco-Loria, M. \& Dumoulin, S. Use of digital image correlation to measure large-strain tensile properties of ductile thermoplastics. Polym. Test. 28, 653-660 (2009).

46. Mzabi, S., Berghezan, D., Roux, S., Hild, F. \& Creton, C. A critical local energy release rate criterion for fatigue fracture of elastomers. J. Polym. Sci. Part B Polym. Phys. 49, 1518-1524 (2011).

47. Hall, M. S., Long, R., Hui, C.-Y. \& Wu, M. Mapping Three-Dimensional Stress and Strain Fields within a Soft Hydrogel Using a Fluorescence Microscope. Biophys. J. 102, 2241-2250 (2012).

48. Liu, W. \& Long, R. Constructing Continuous Strain and Stress Fields From Spatially Discrete Displacement Data in Soft Materials. J. Appl. Mech. 83, 011006 (2015).

49. Belytschko, T., Lu, Y. Y. \& Gu, L. Element-free Galerkin methods. Int. J. Numer. Methods Eng. 37, 229-256 (1994).

50. Xinzeng, F., Matthew, S. H., Mingming, W. \& Chung-Yuen, H. An adaptive algorithm for tracking 3D bead displacements: application in biological experiments. Meas. Sci. Technol. 25, 55701 (2014).

51. Patel, M., Leggett, S. E., Landauer, A. K., Wong, I. Y. \& Franck, C. Rapid, topology-based particle tracking for high-resolution measurements of large complex 3D motion fields. Sci. Rep. 8, 5581 (2018).

52. Boltyanskiy, R., Merrill, J. W. \& Dufresne, E. R. Tracking particles with large displacements using energy minimization. Soft Matter 13, 2201-2206 (2017).

53. Gossweiler, G. R. et al. Mechanochemically Active Soft Robots. ACS Appl. Mater. Interfaces 7, 22431-22435 (2015). 
54. Rivlin, R. S. \& Thomas, A. G. Rupture of rubber. I. Characteristic energy for tearing. J. Polym. Sci. 10, 291-318 (1953).

55. Yeoh, O. H. Analysis of deformation and fracture of 'pure shear' rubber testpiece. Plast. Rubber Compos. 30, 389-397 (2001).

56. Knowles, J. K. The finite anti-plane shear field near the tip of a crack for a class of incompressible elastic solids. Int. J. Fract. 13, 611-639 (1977).

57. Geubelle, P. H. \& Knauss, W. G. Finite strains at the tip of a crack in a sheet of hyperelastic material: I. Homogeneous case. J. Elast. 35, 61-98 (1994).

58. Long, R., Krishnan, V. R. \& Hui, C. Y. Finite strain analysis of crack tip fields in incompressible hyperelastic solids loaded in plane stress. J. Mech. Phys. Solids 59, $672-695$ (2011).

59. de Gennes, P. G. Soft Adhesives †. Langmuir 12, 4497-4500 (1996).

60. Saulnier, F., Ondarçuhu, T., Aradian, A. \& Raphaël, E. Adhesion between a Viscoelastic Material and a Solid Surface. Macromolecules 37, 1067-1075 (2004).

61. Tanaka, Y. et al. Determination of Fracture Energy of High Strength Double Network Hydrogels. J. Phys. Chem. B 109, 11559-11562 (2005).

62. Baumberger, T., Caroli, C. \& Martina, D. Fracture of a biopolymer gel as a viscoplastic disentanglement process. Eur. Phys. J. E 21, 81-89 (2006).

63. Luo, F. et al. Crack Blunting and Advancing Behaviors of Tough and Self-healing Polyampholyte Hydrogel. Macromolecules 47, 6037-6046 (2014).

64. Sun, T. L. et al. Bulk Energy Dissipation Mechanism for the Fracture of Tough and Self-Healing Hydrogels. Macromolecules 50, 2923-2931 (2017).

65. Mayumi, K., Guo, J., Narita, T., Hui, C. Y. \& Creton, C. Fracture of dual crosslink gels with permanent and transient crosslinks. Extrem. Mech. Lett. 6, 52-59 (2016).

66. Case, J. C., White, E. L. \& Kramer, R. K. Soft Material Characterization for Robotic Applications. Soft Robot. 2, 80-87 (2015).

67. Maskarinec, S.A., Franck, C., Tirrell, D.A., Ravichandran, G. Quantifying cellular 
traction forces in three dimensions, Proc. Nat. Acad. Sci., 106, 22108-22113 (2009). 
(a) Undeformed Configuration
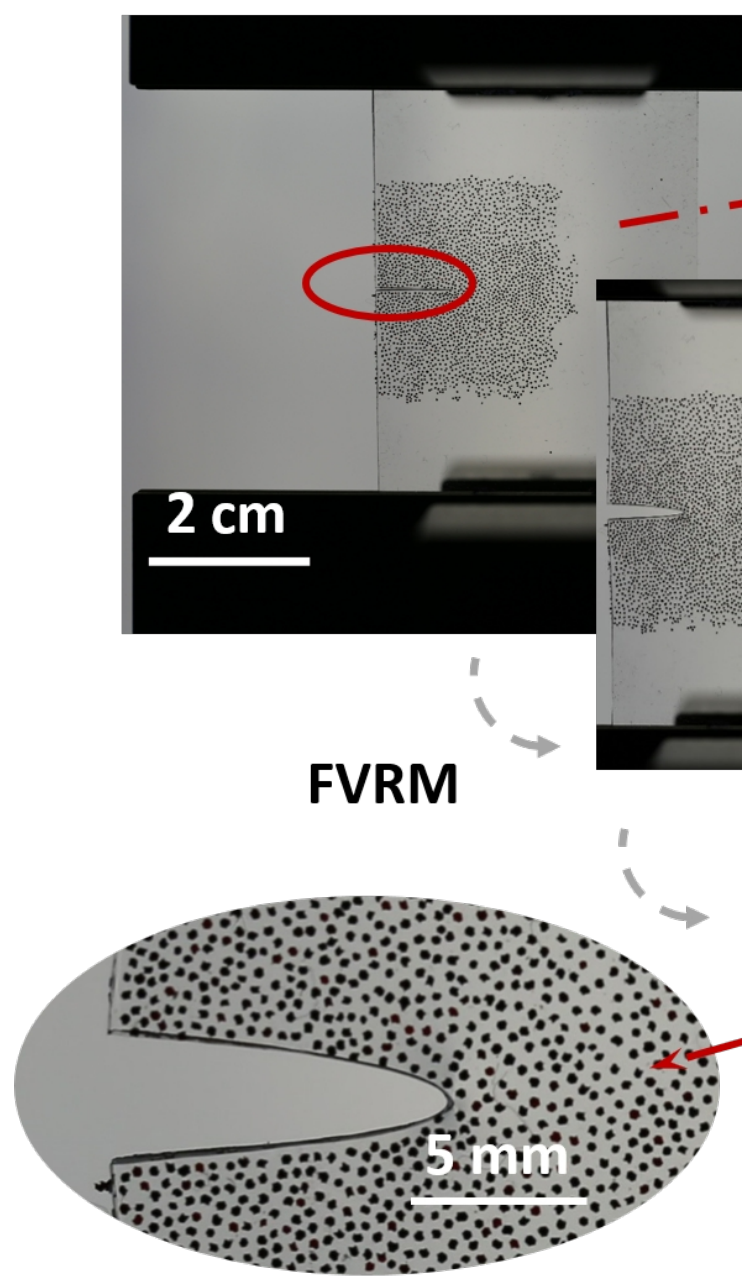

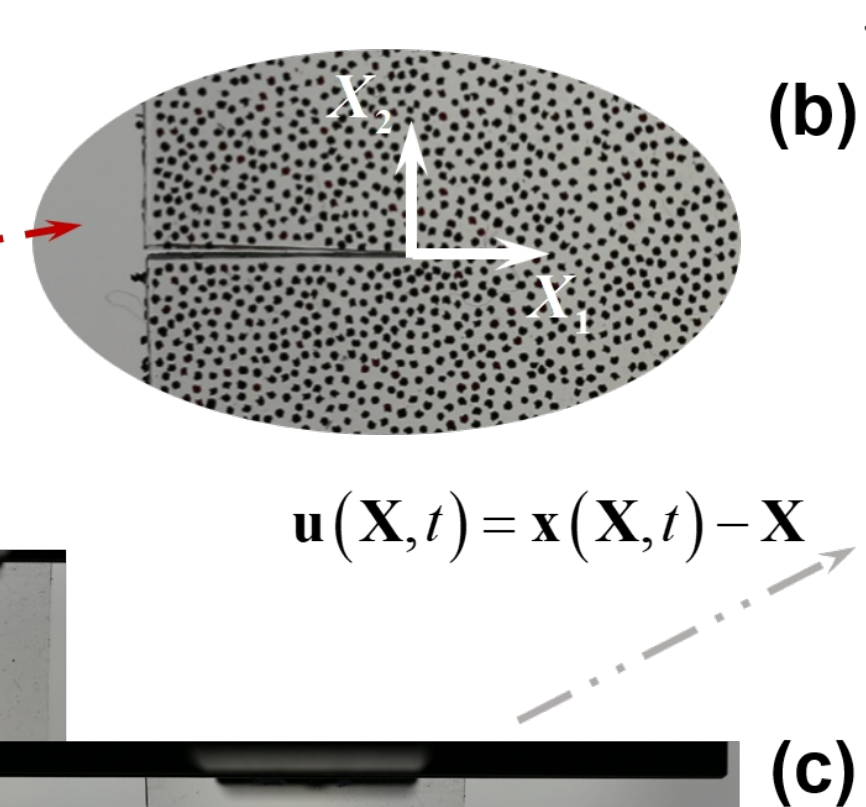

Trajectories of Tracers

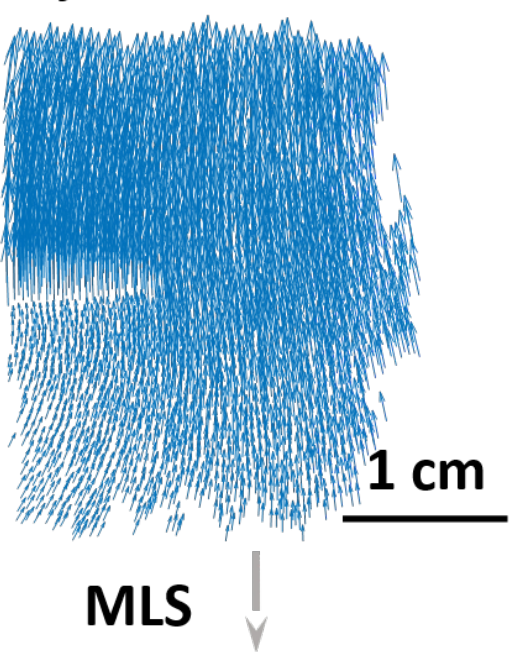

(c) Hencky Strain $\mathbf{e}_{22}$

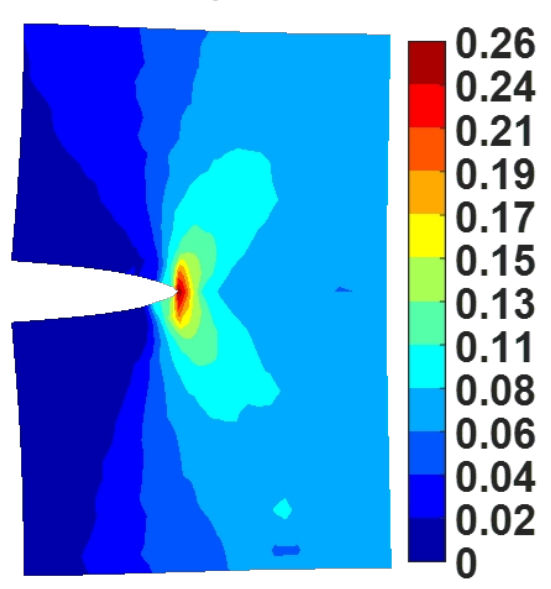

Deformed Configuration 
(a)

Undeformed
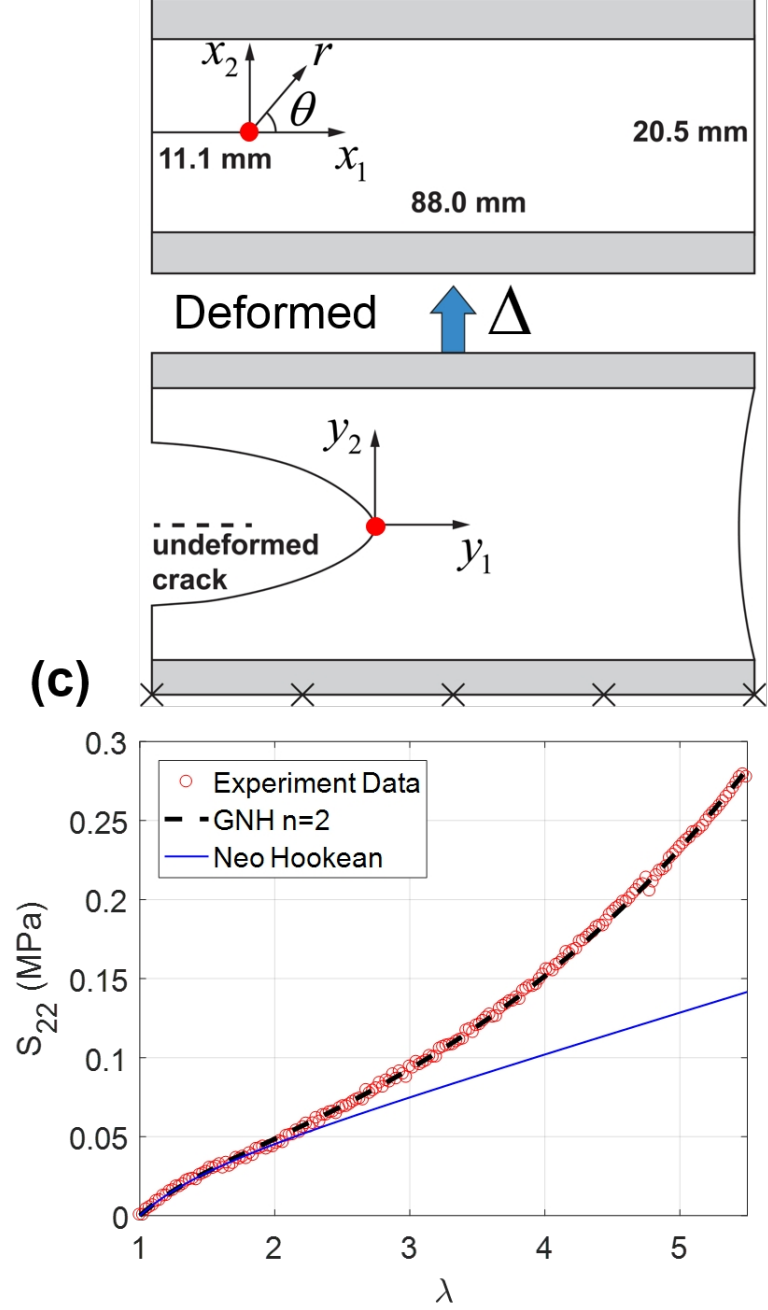

(e)

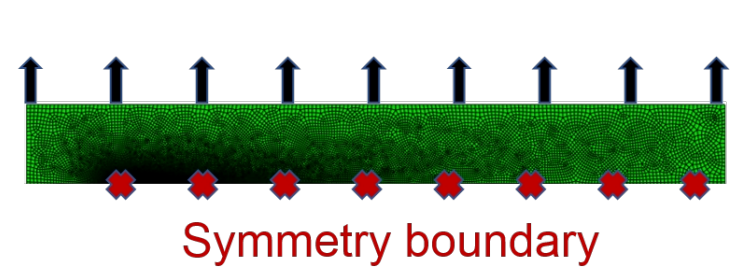

(b)

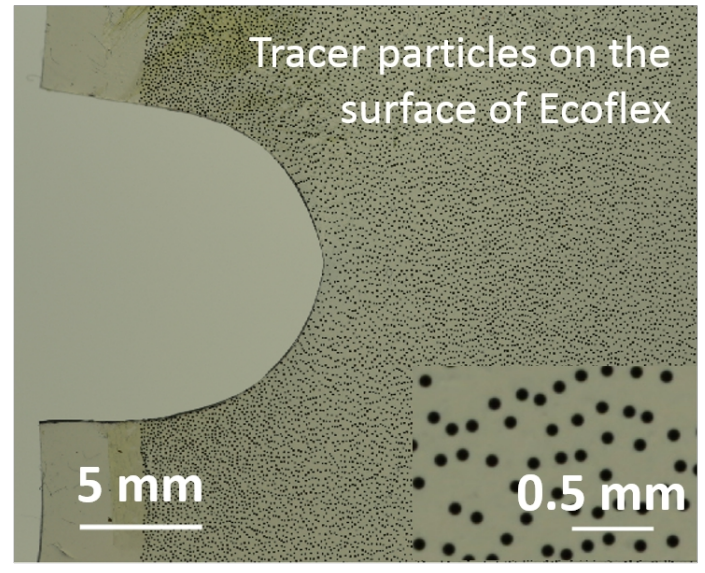

(d)
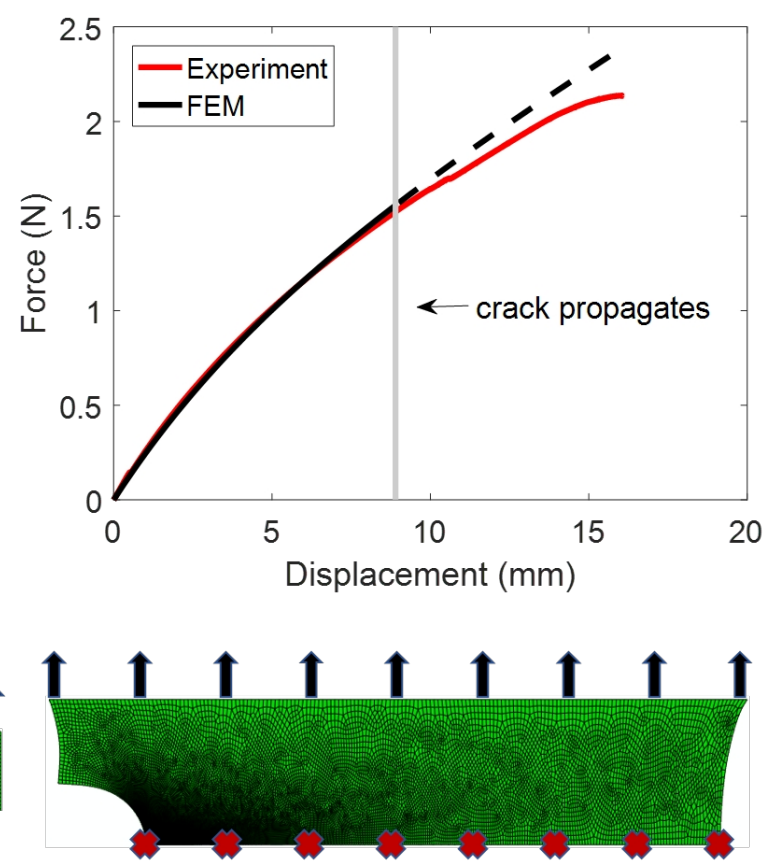
(a)

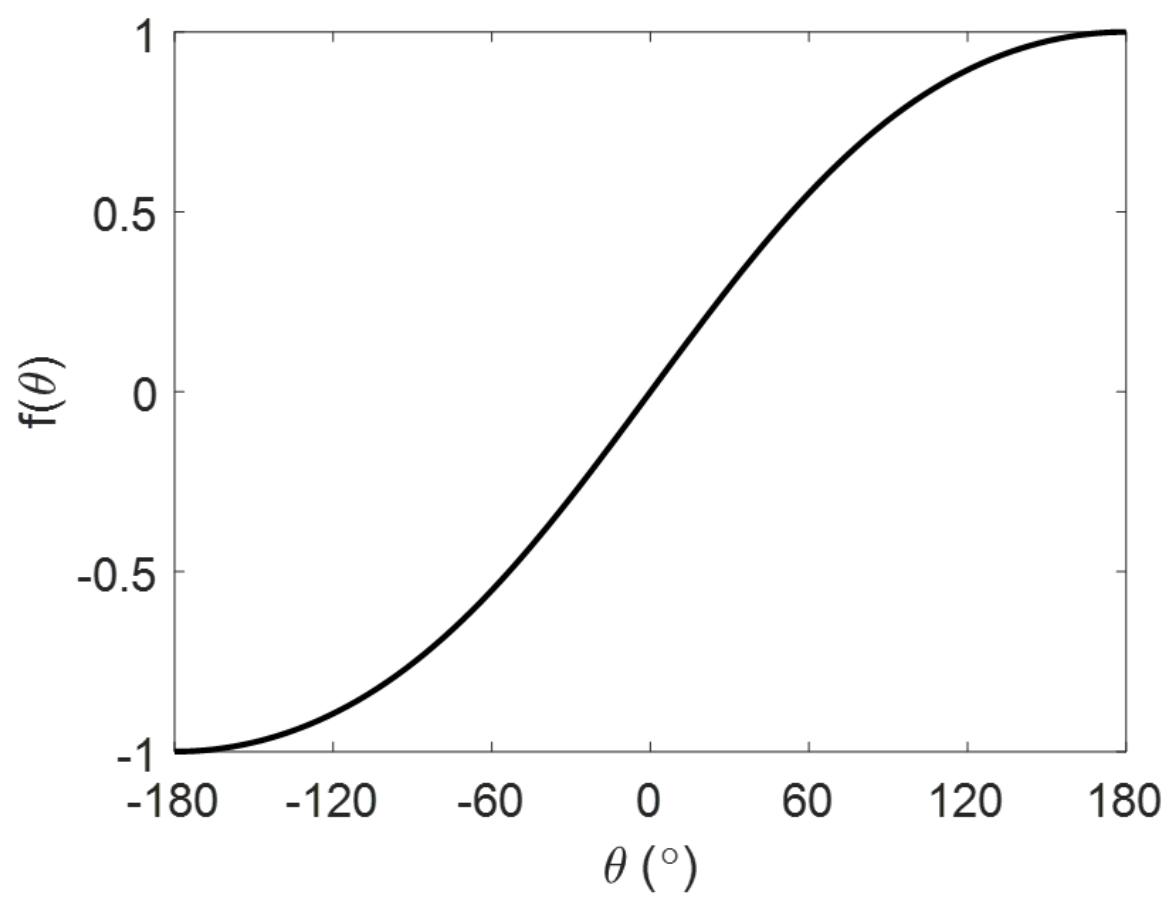

(b)

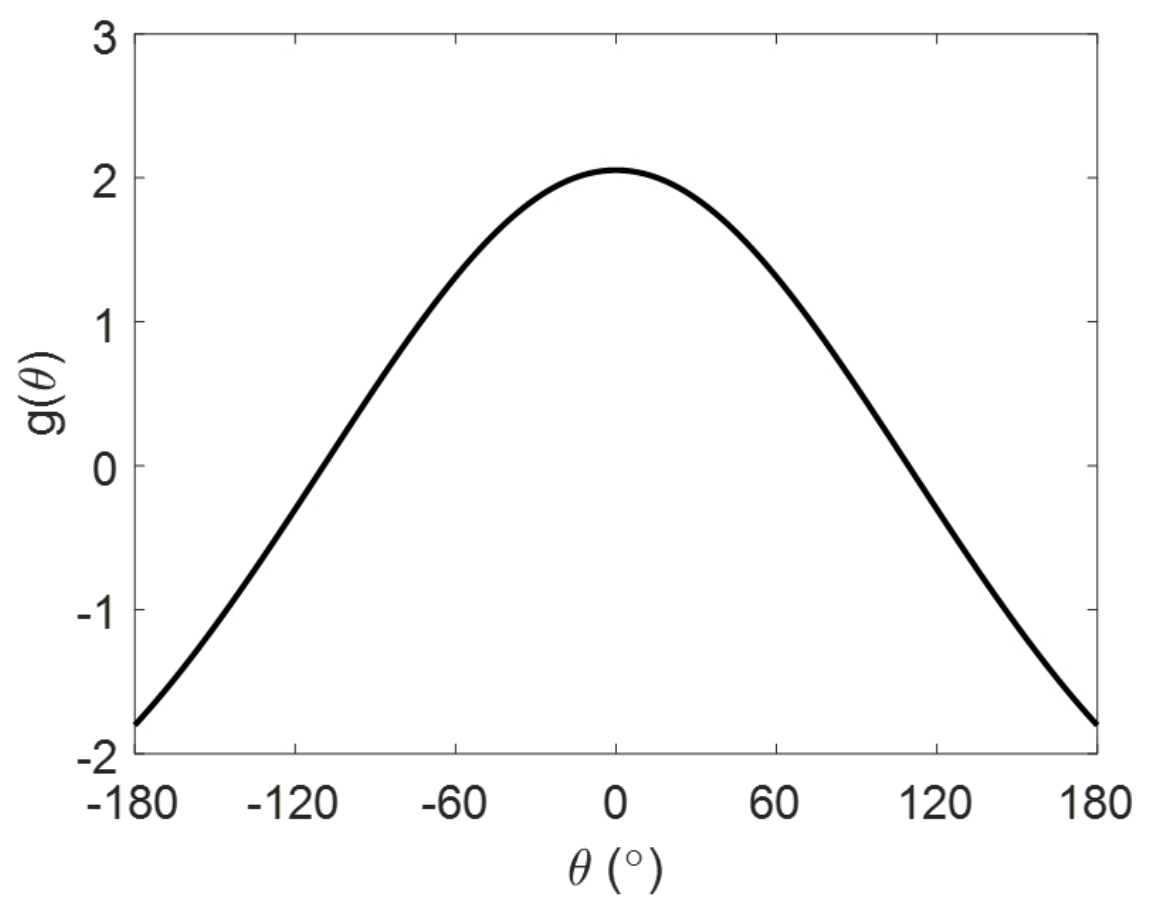


(a)

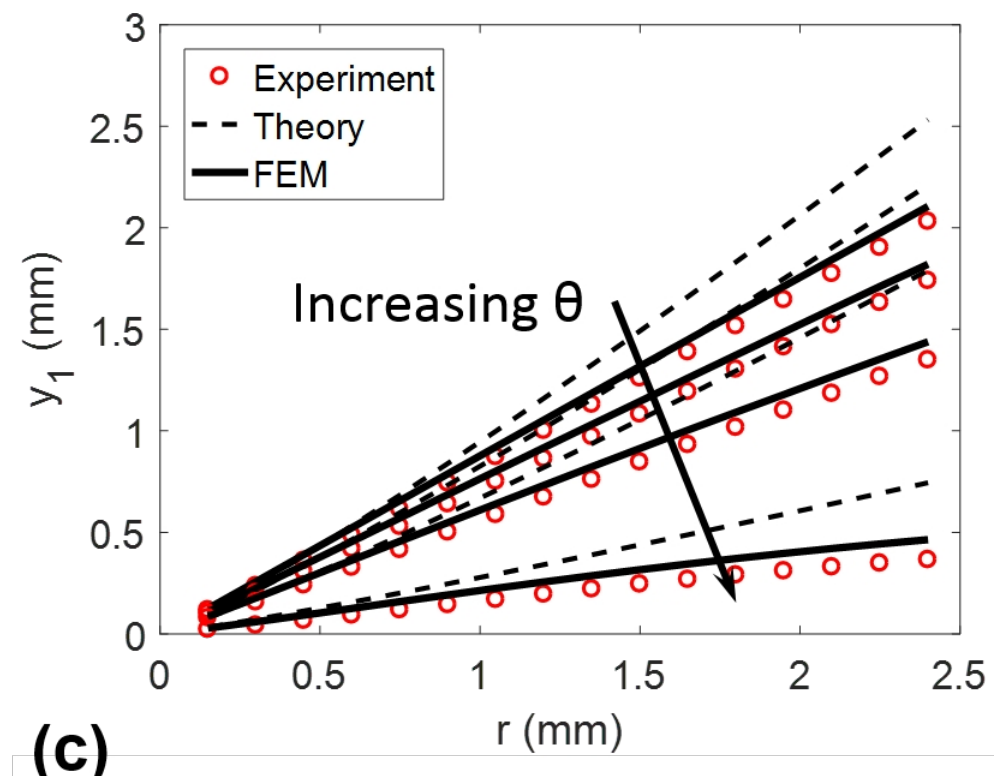

(c)

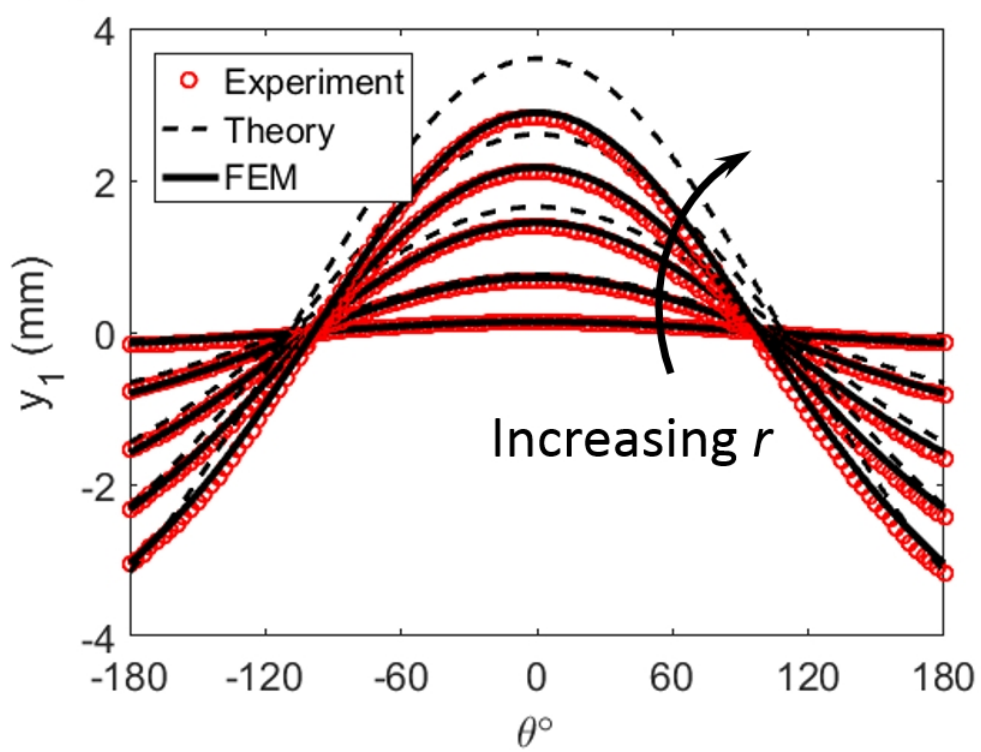

(b)
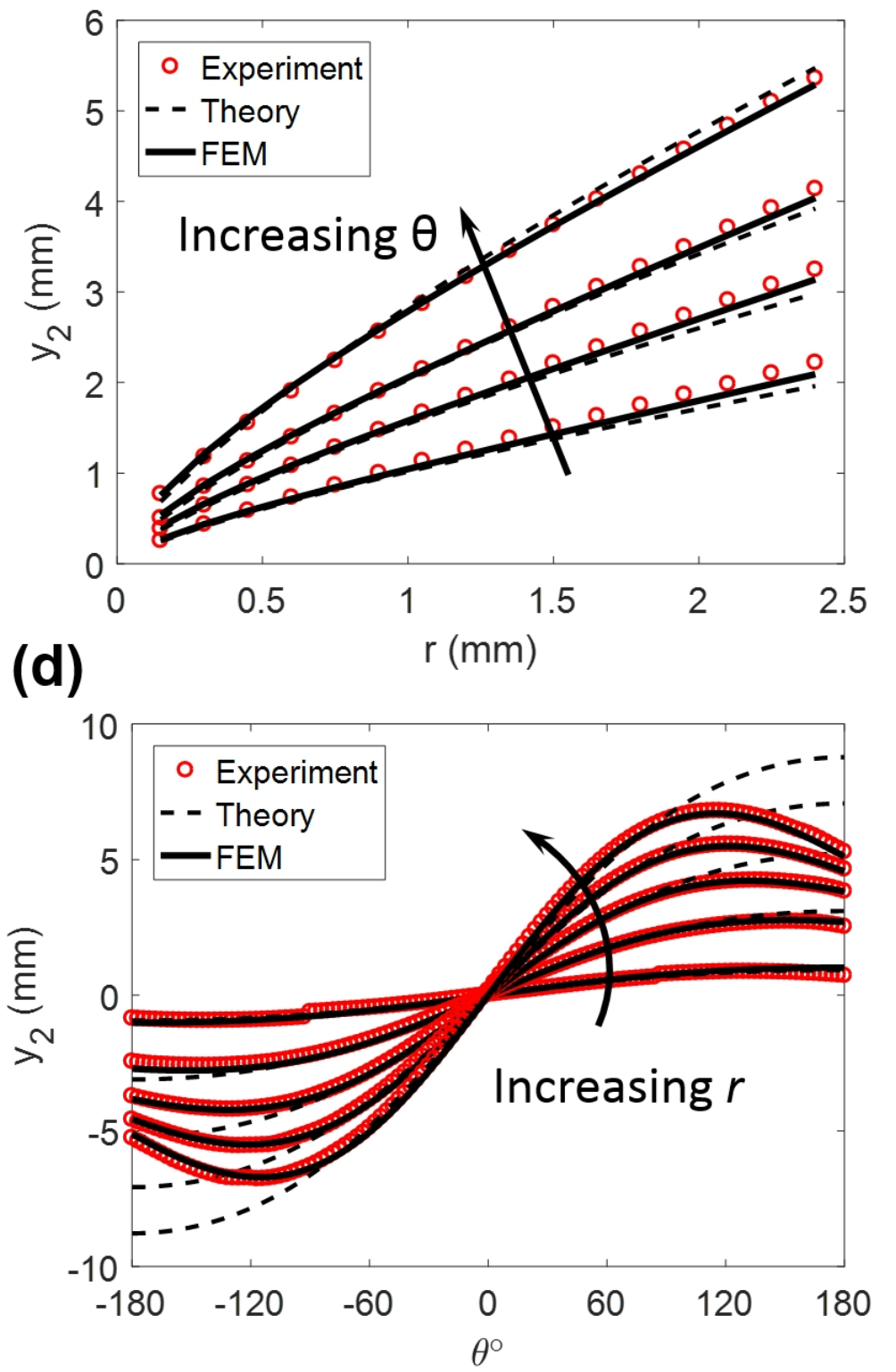
(a)

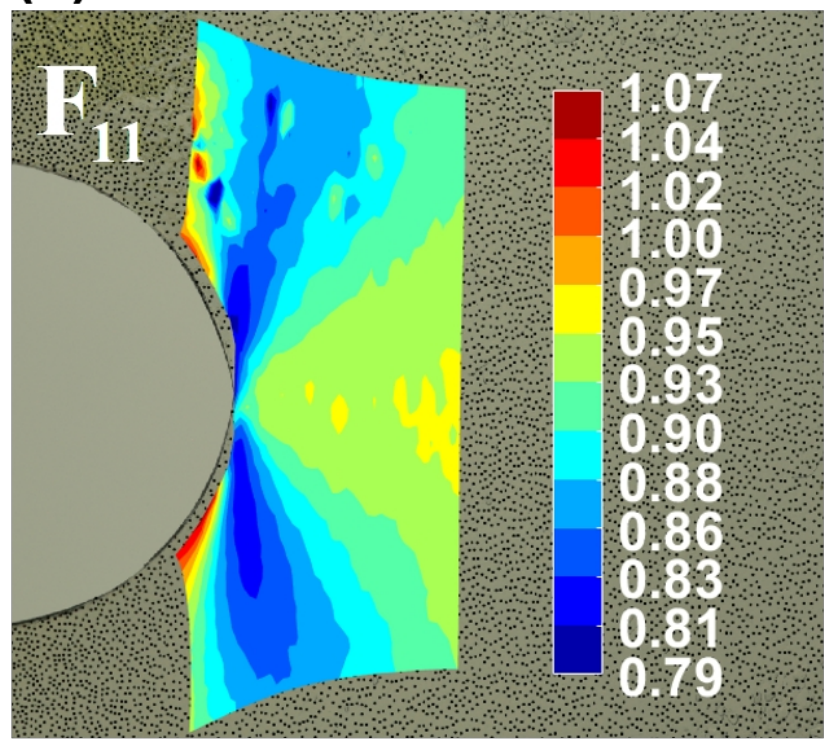

(c)

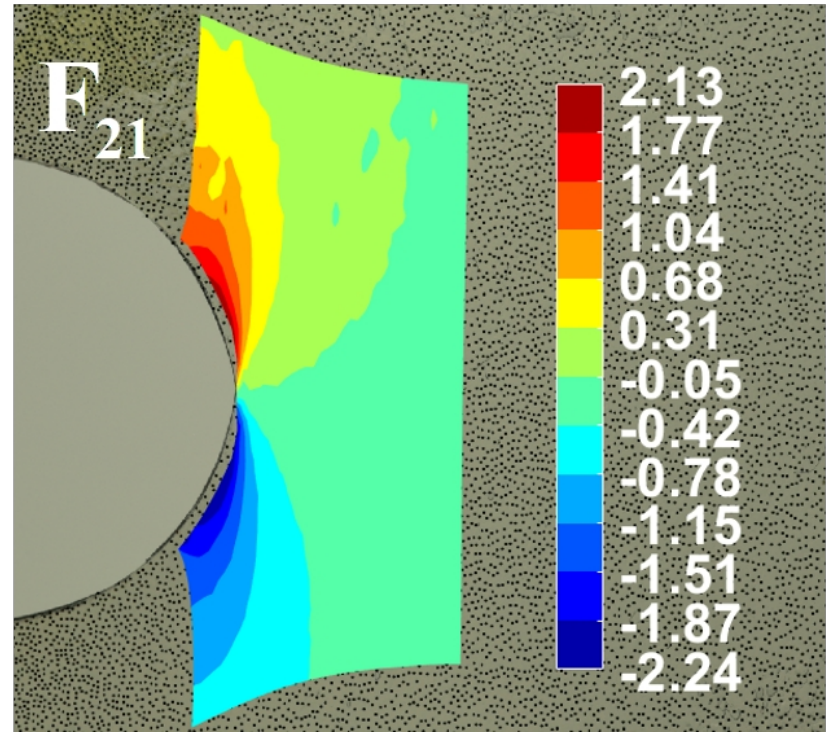

(b)

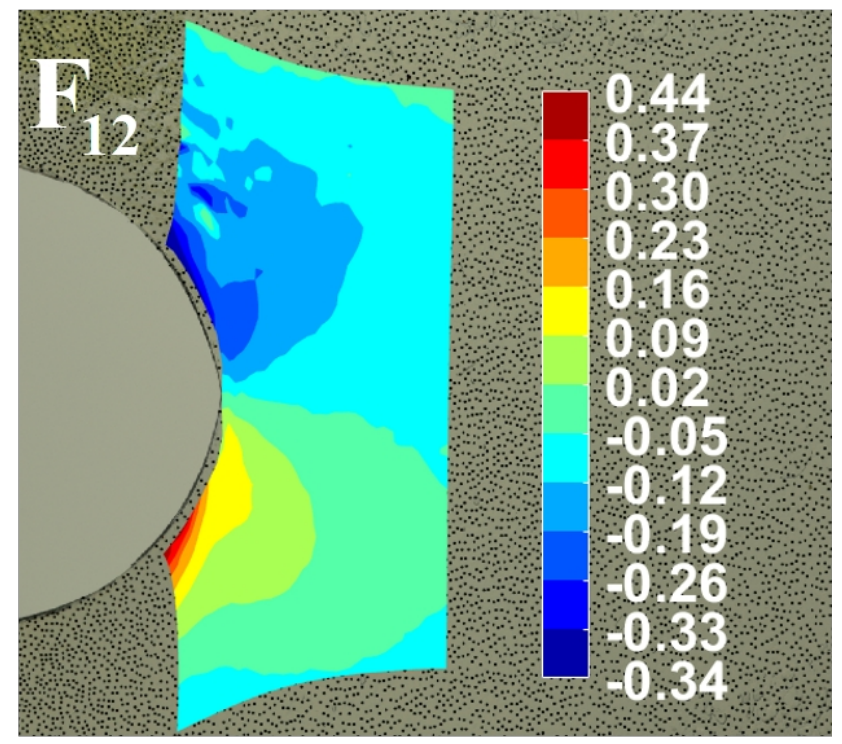

(d)

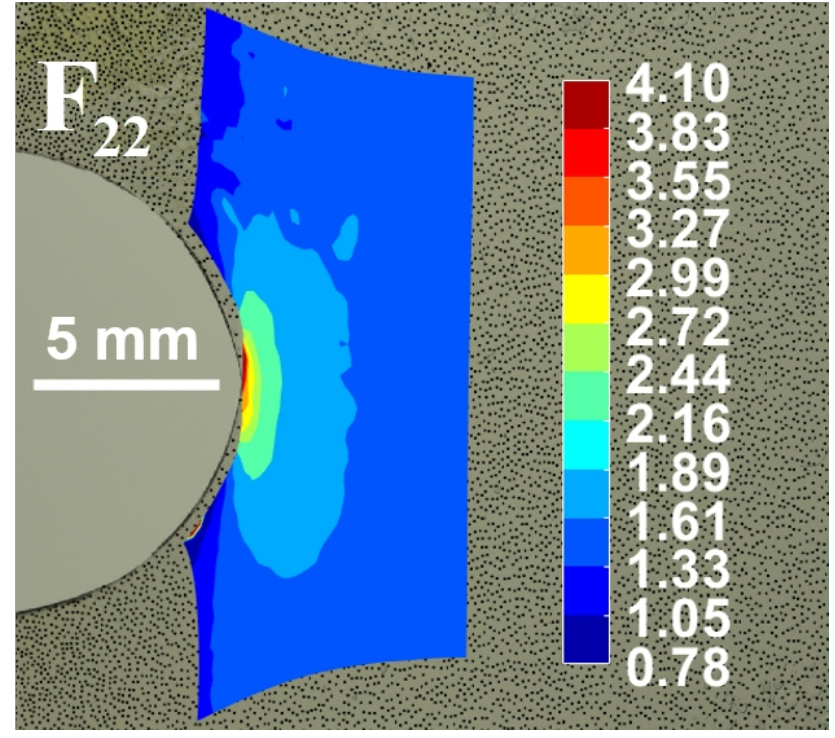


(a)

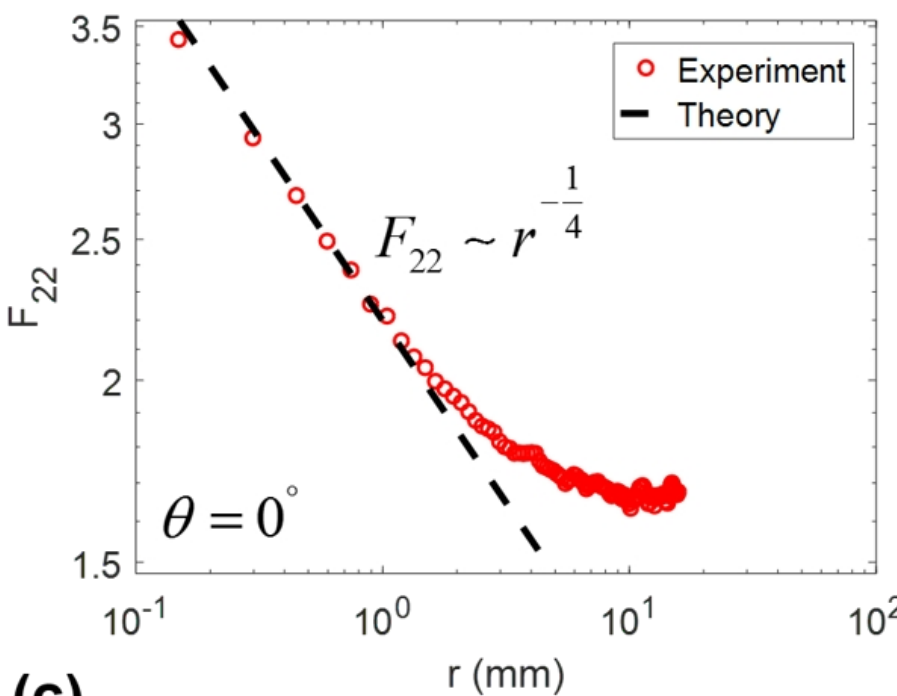

(c)

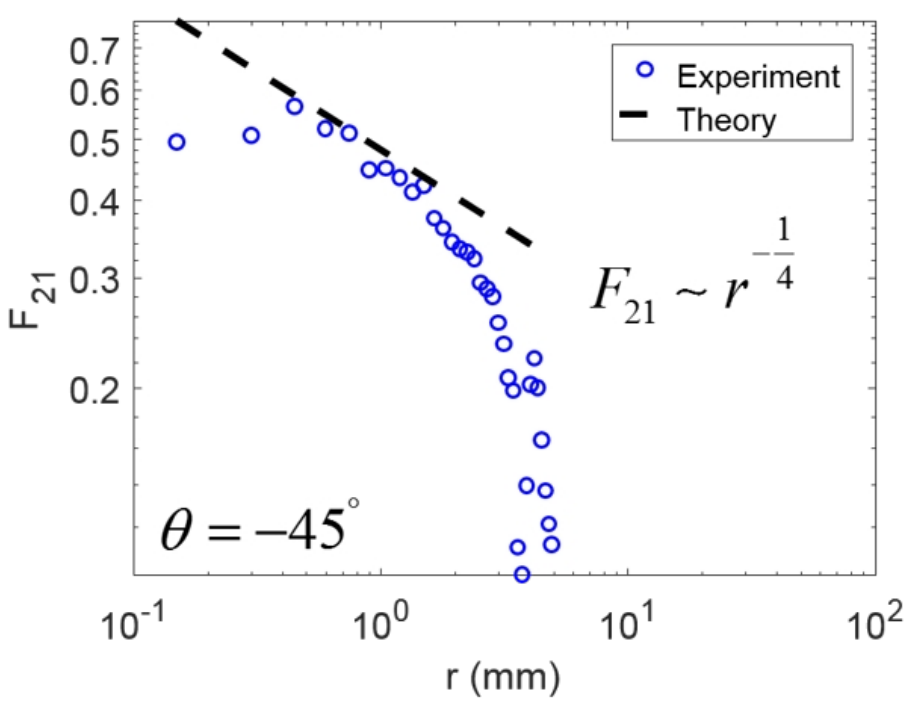

(b)
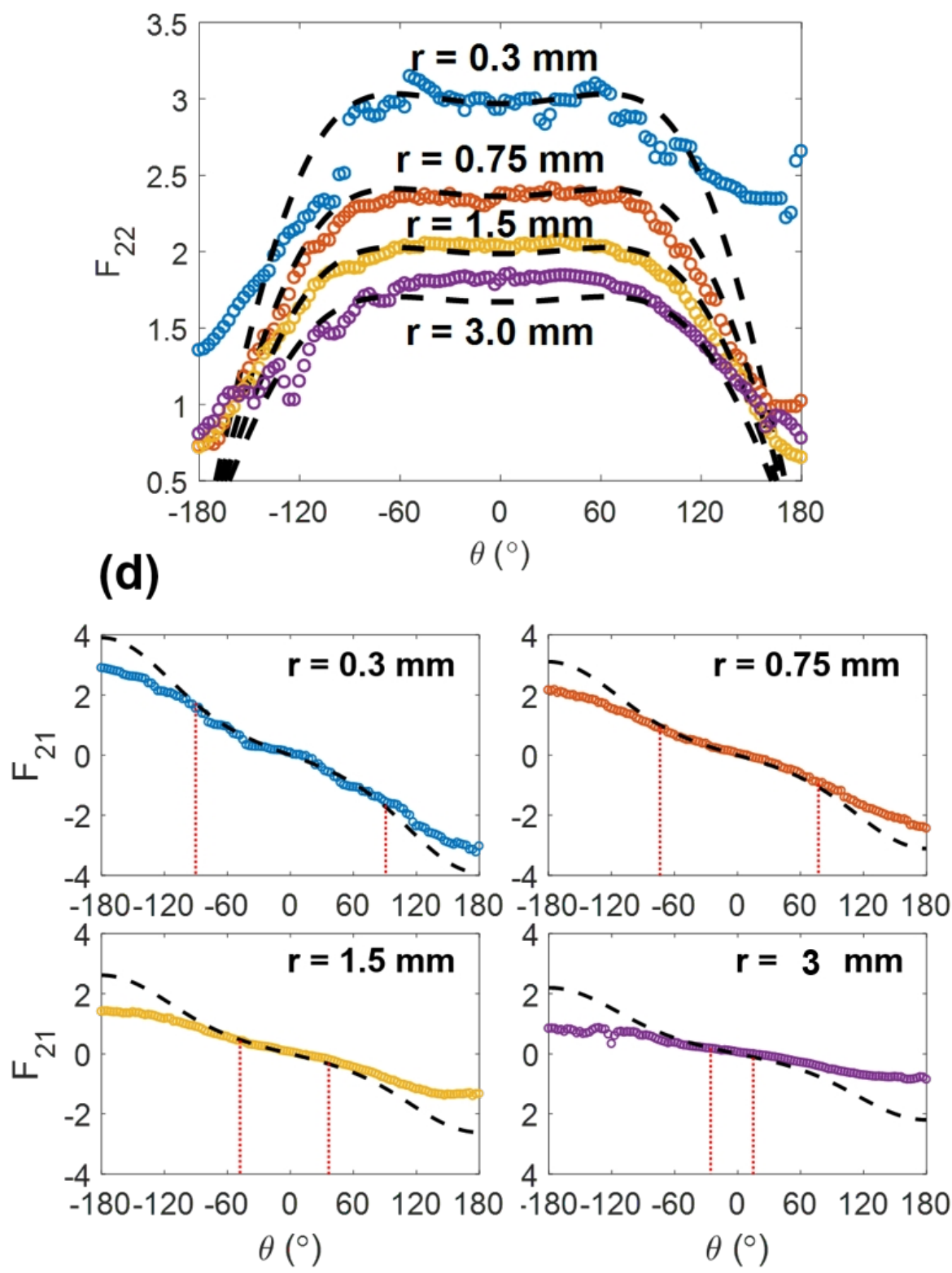
(a)

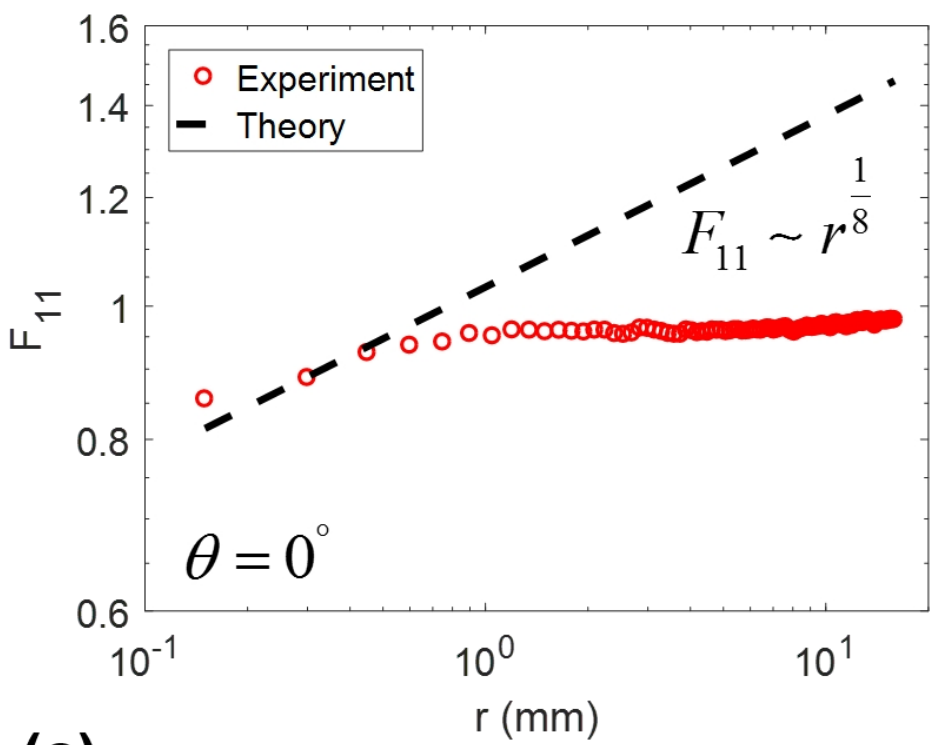

(c)

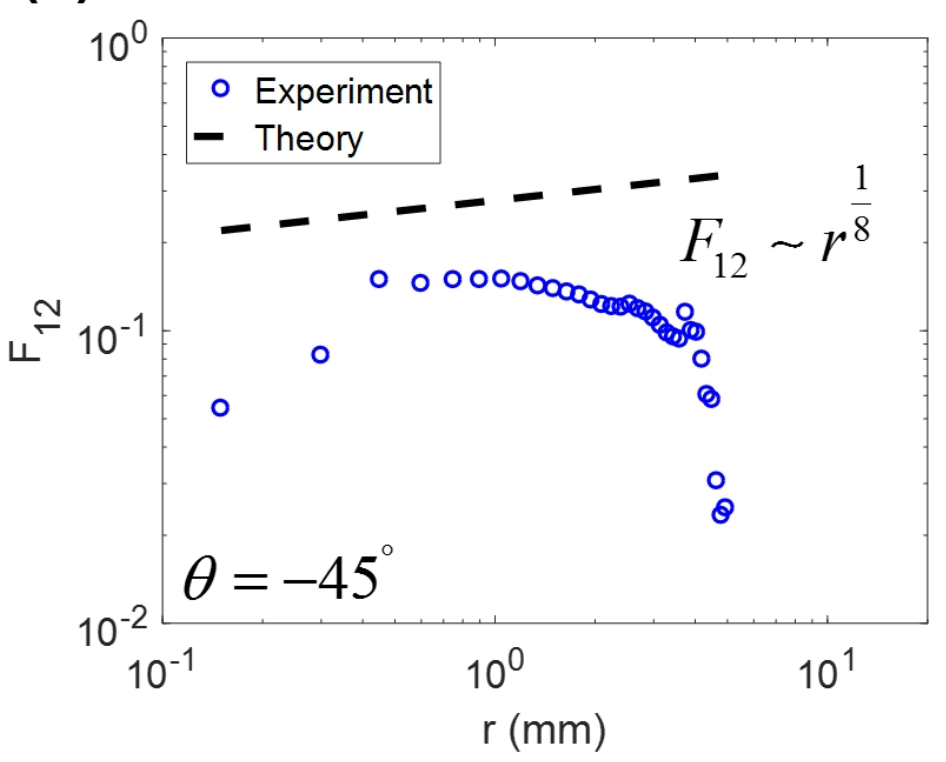

(b)

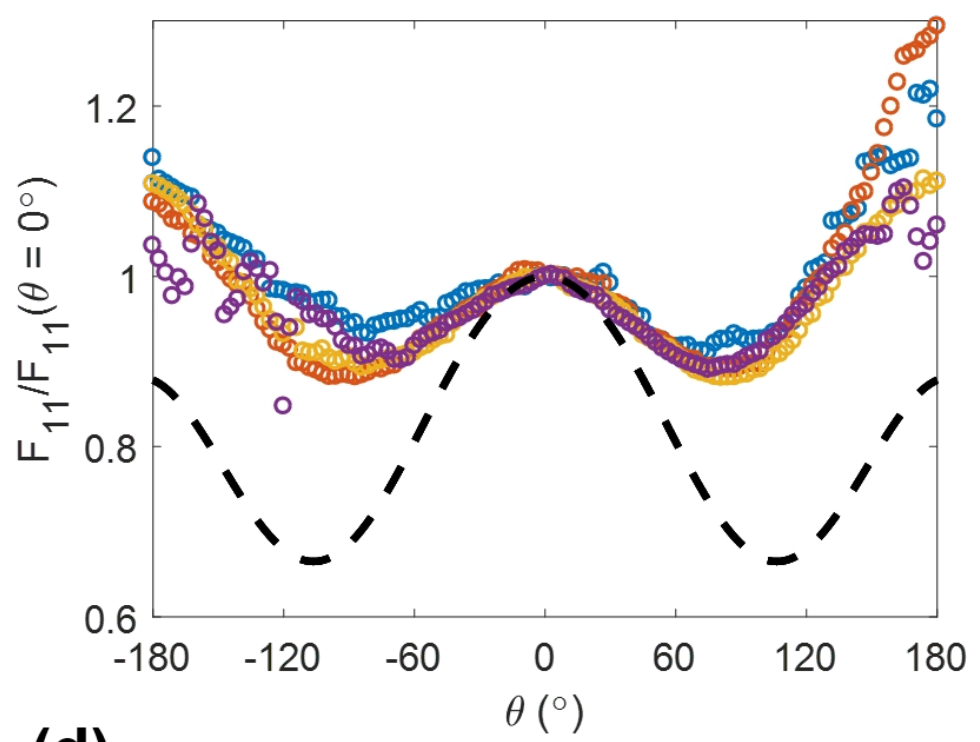

(d)

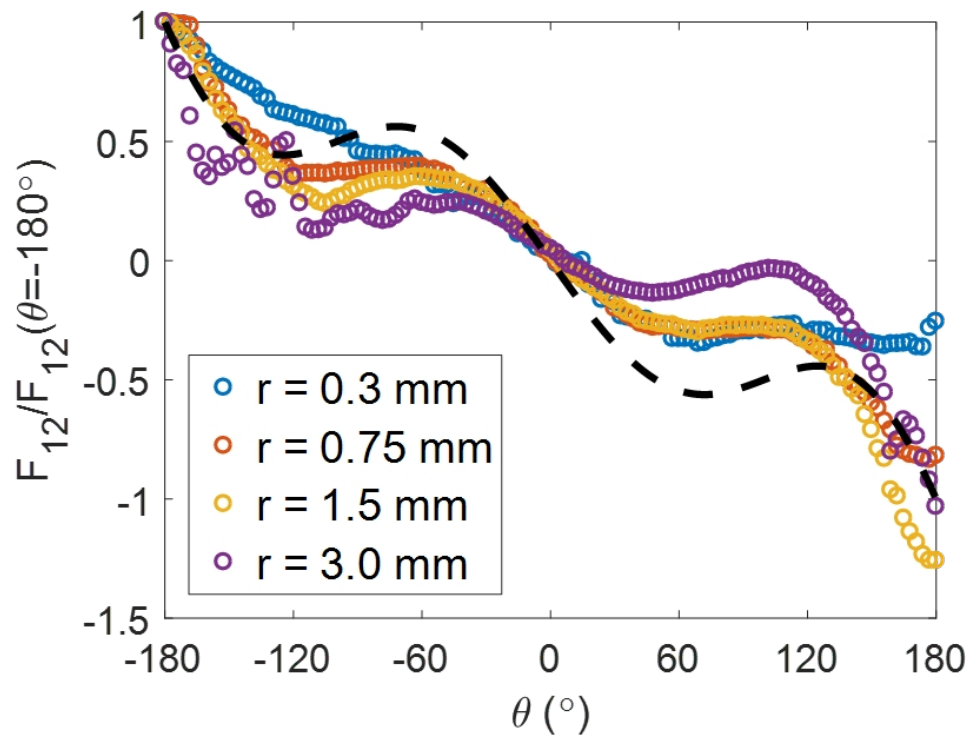


(a)

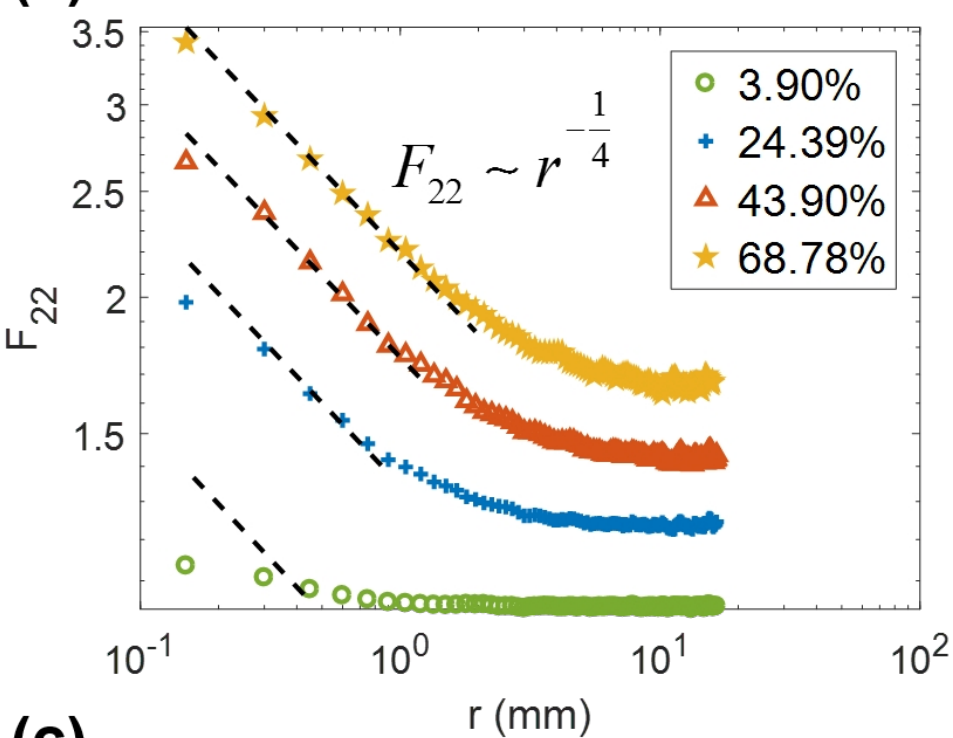

(c)

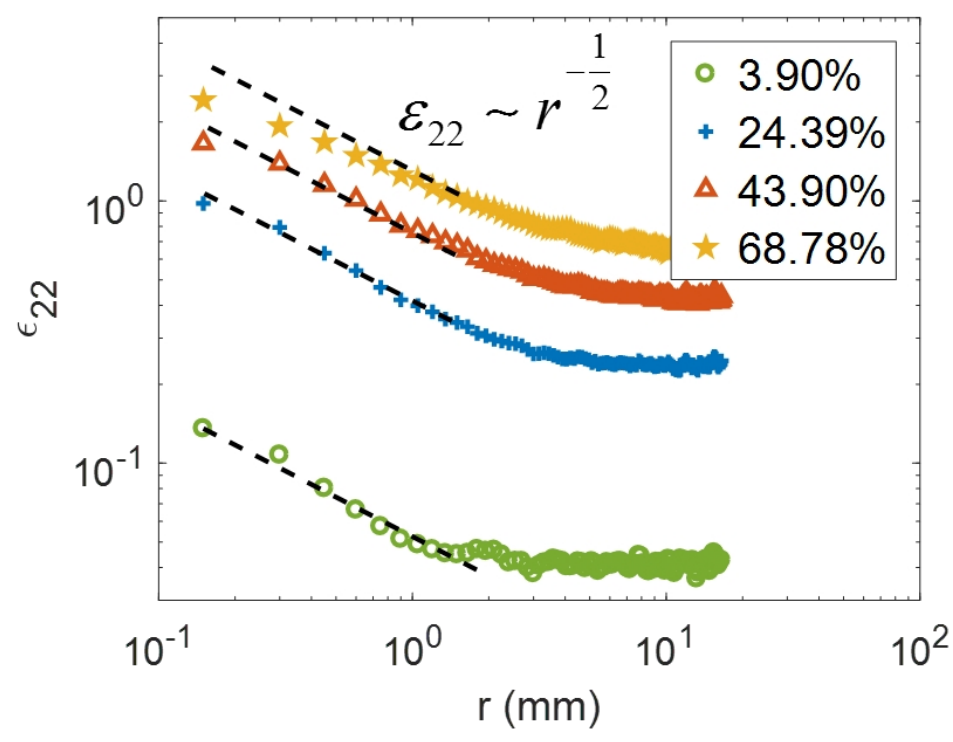

(b)
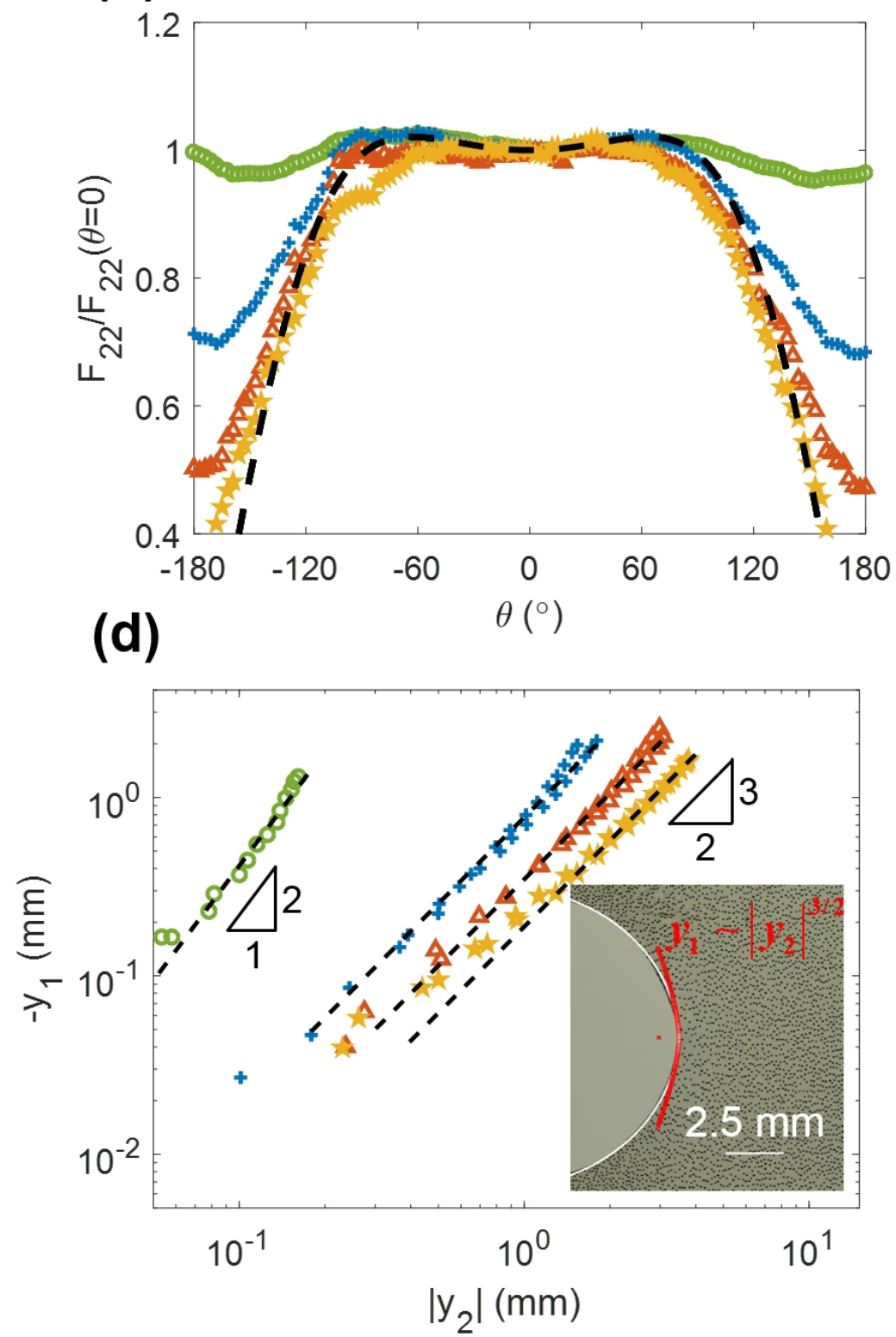
(a)
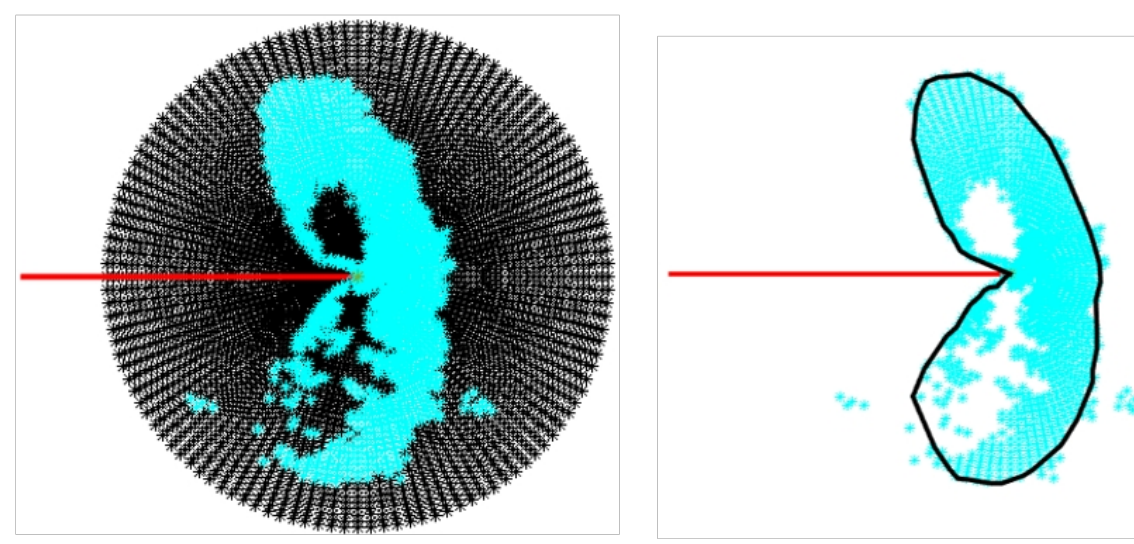

(c)
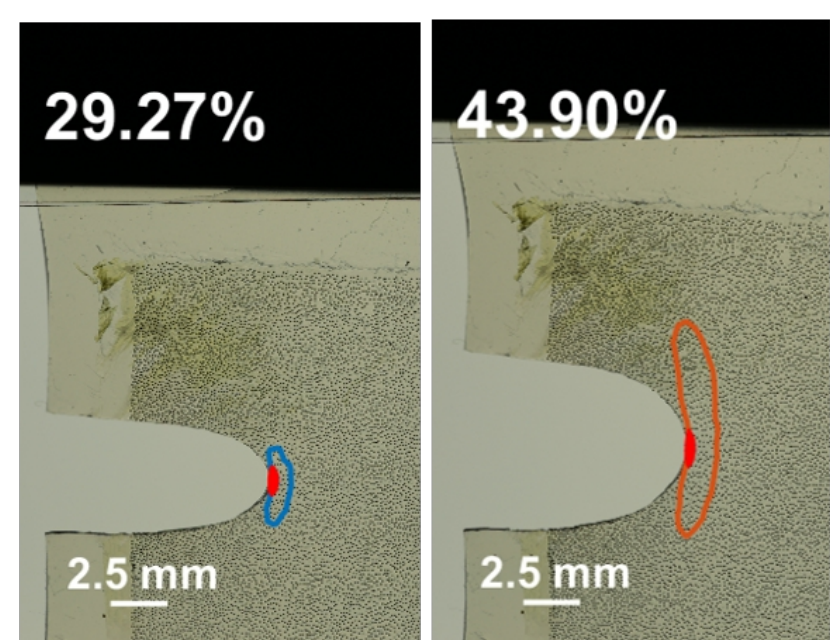

(b)
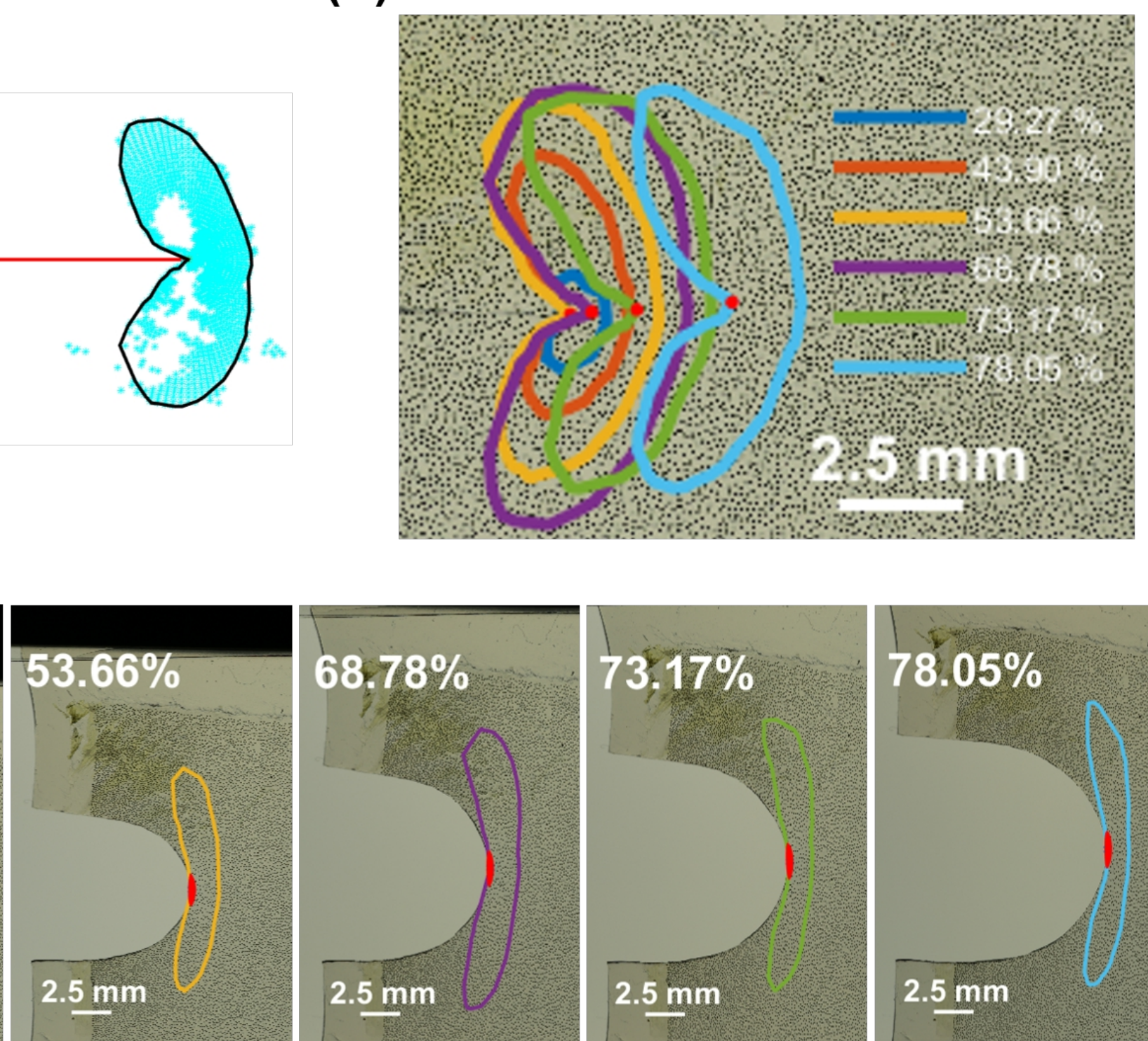

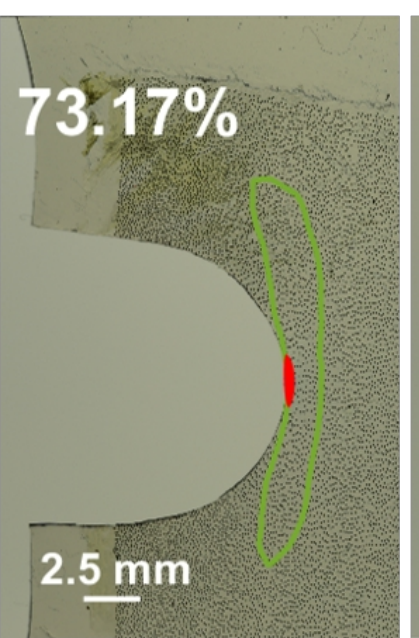

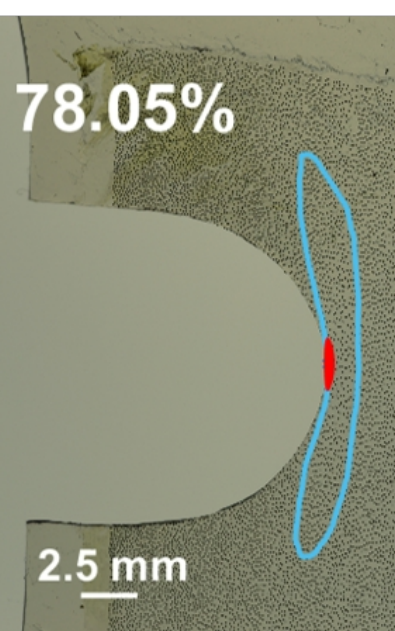


(a)

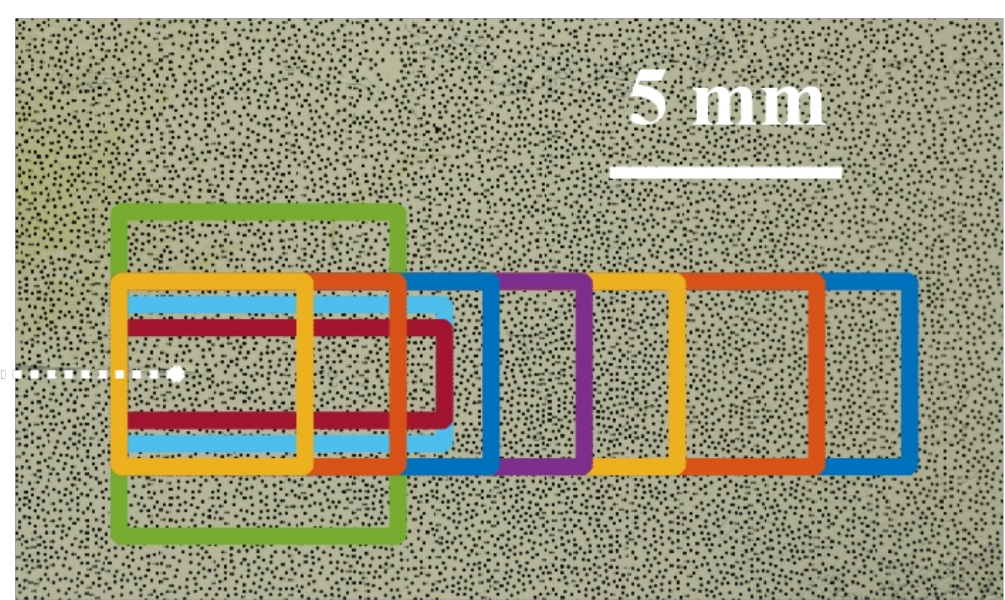

(b)

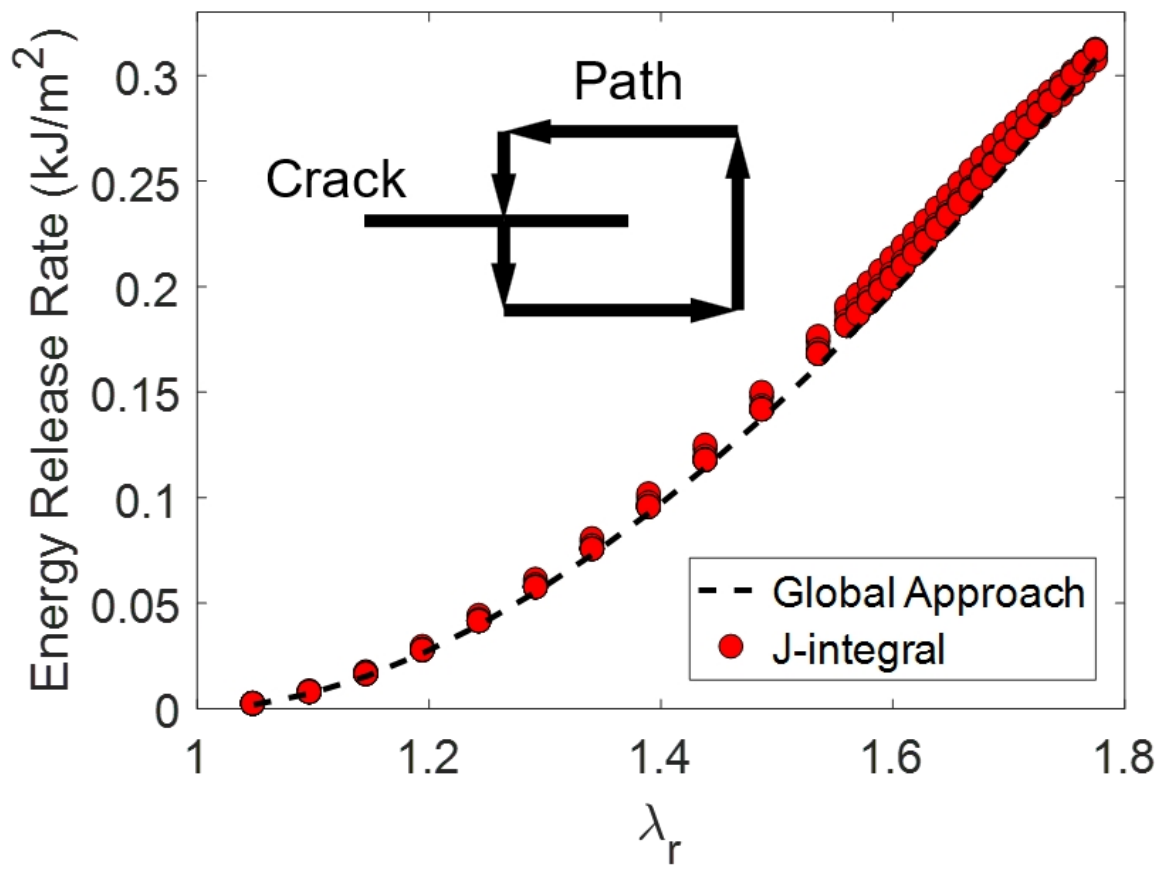

(c)

$\lambda_{r}=1.10$
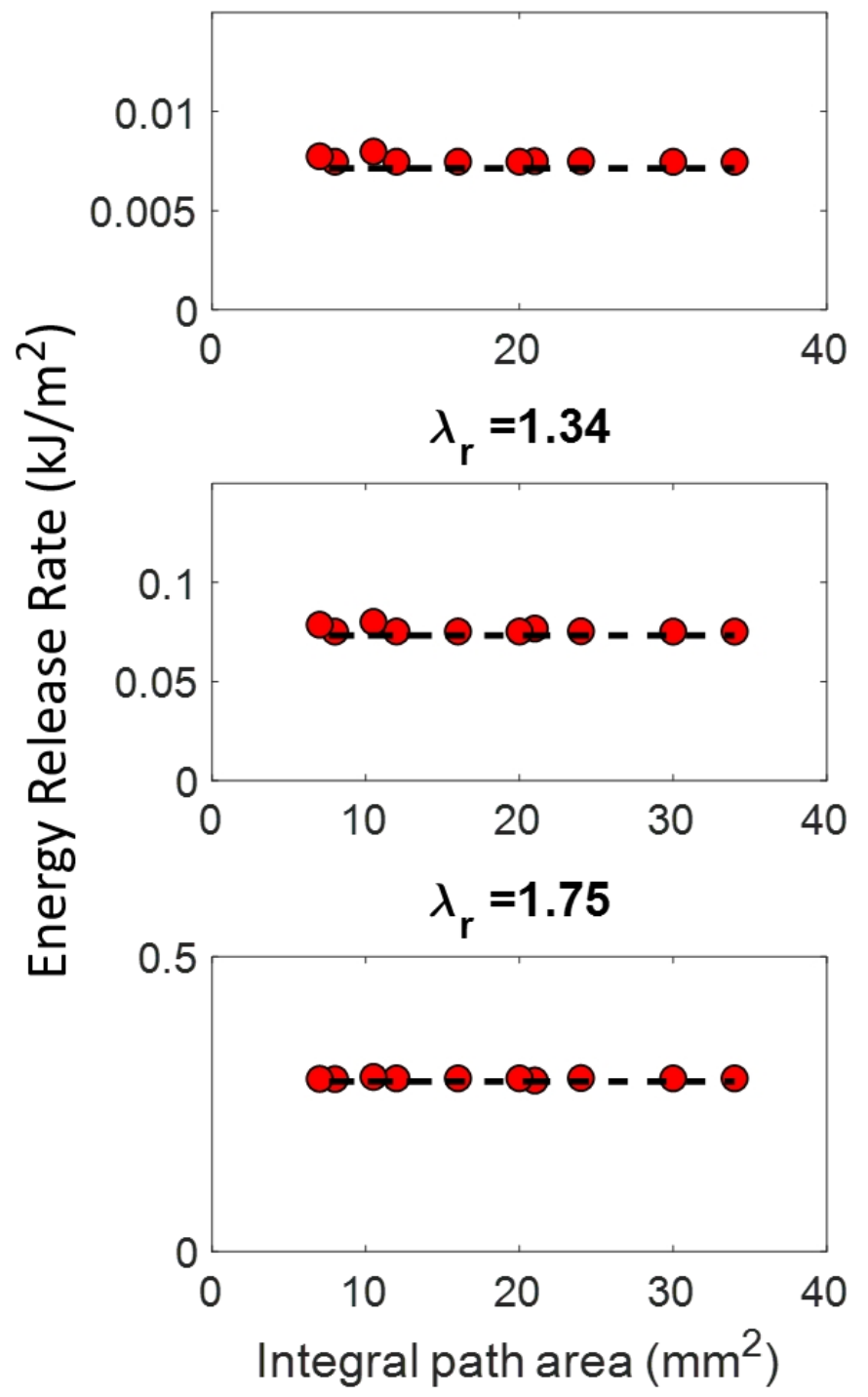
(a)
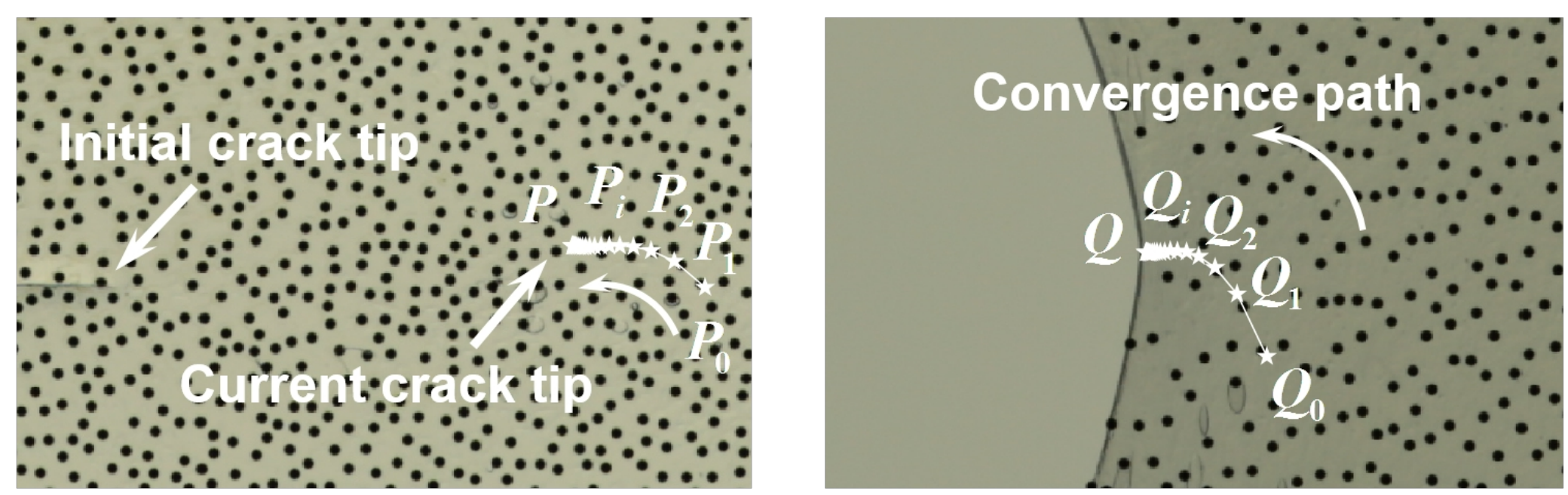

(b)

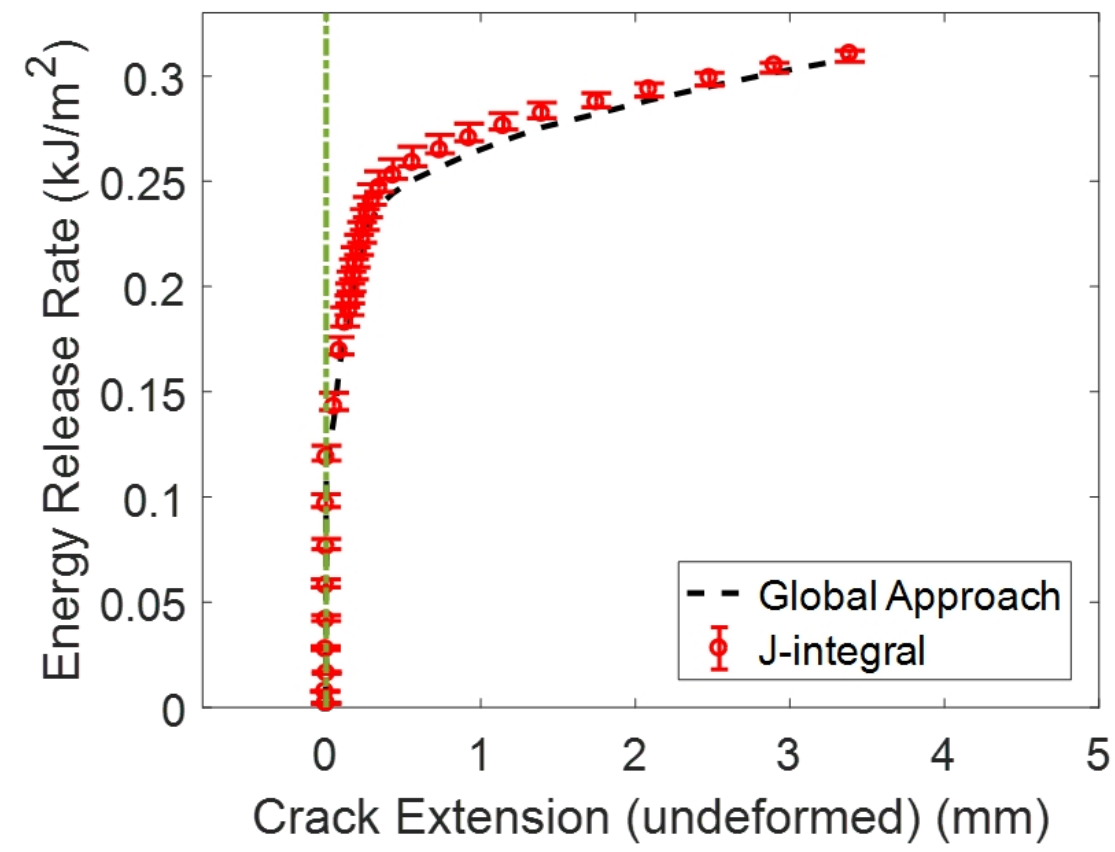

(c)

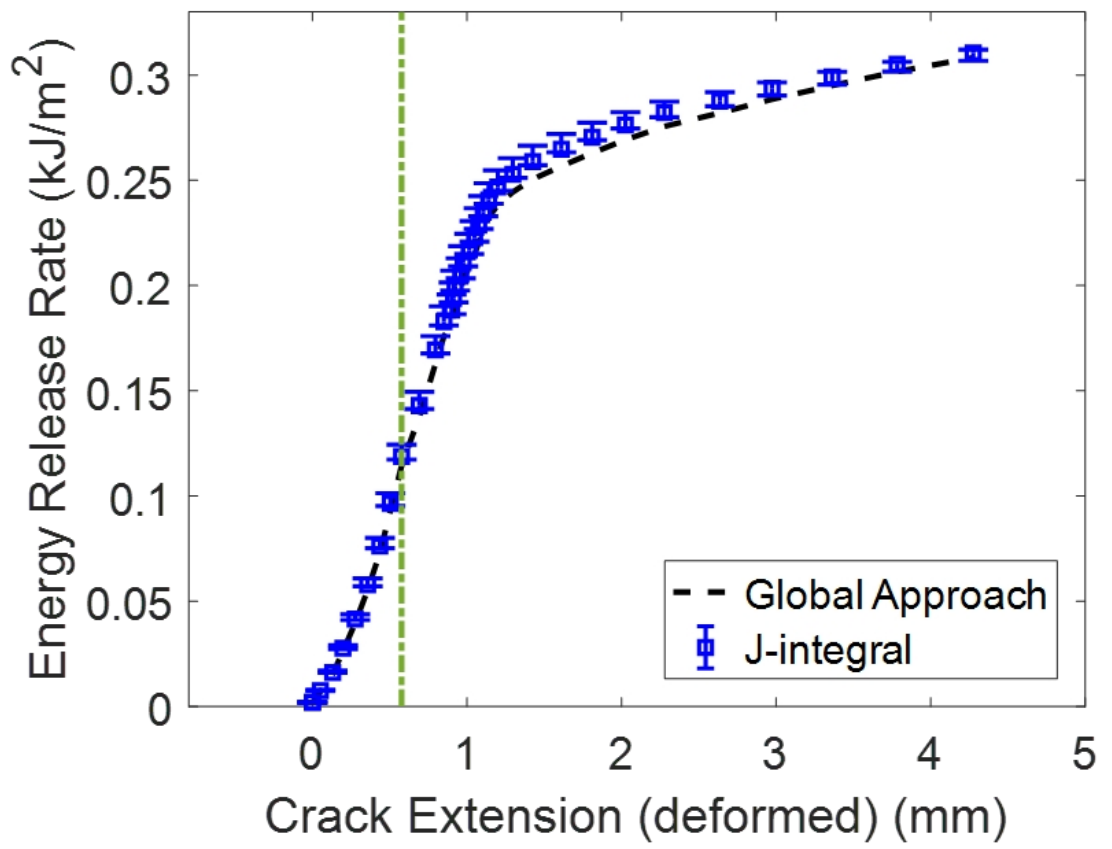


(a)

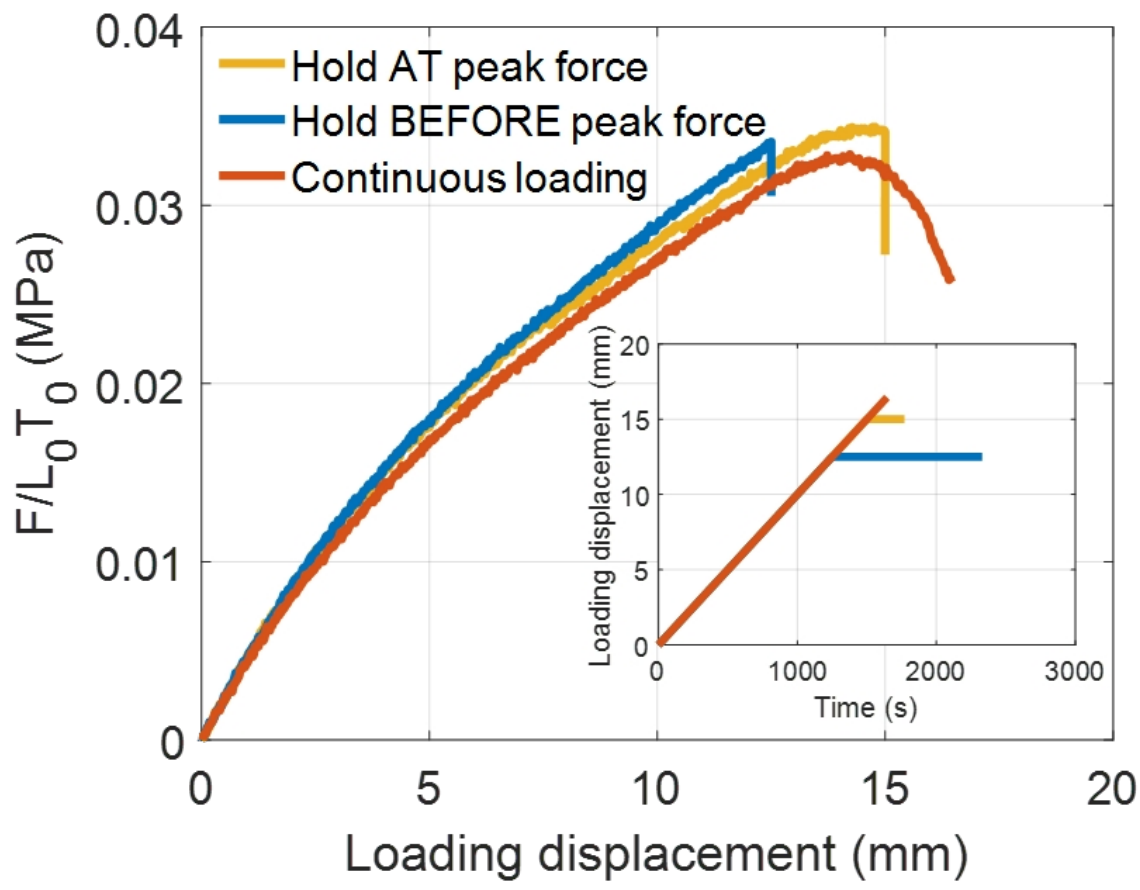

(b)

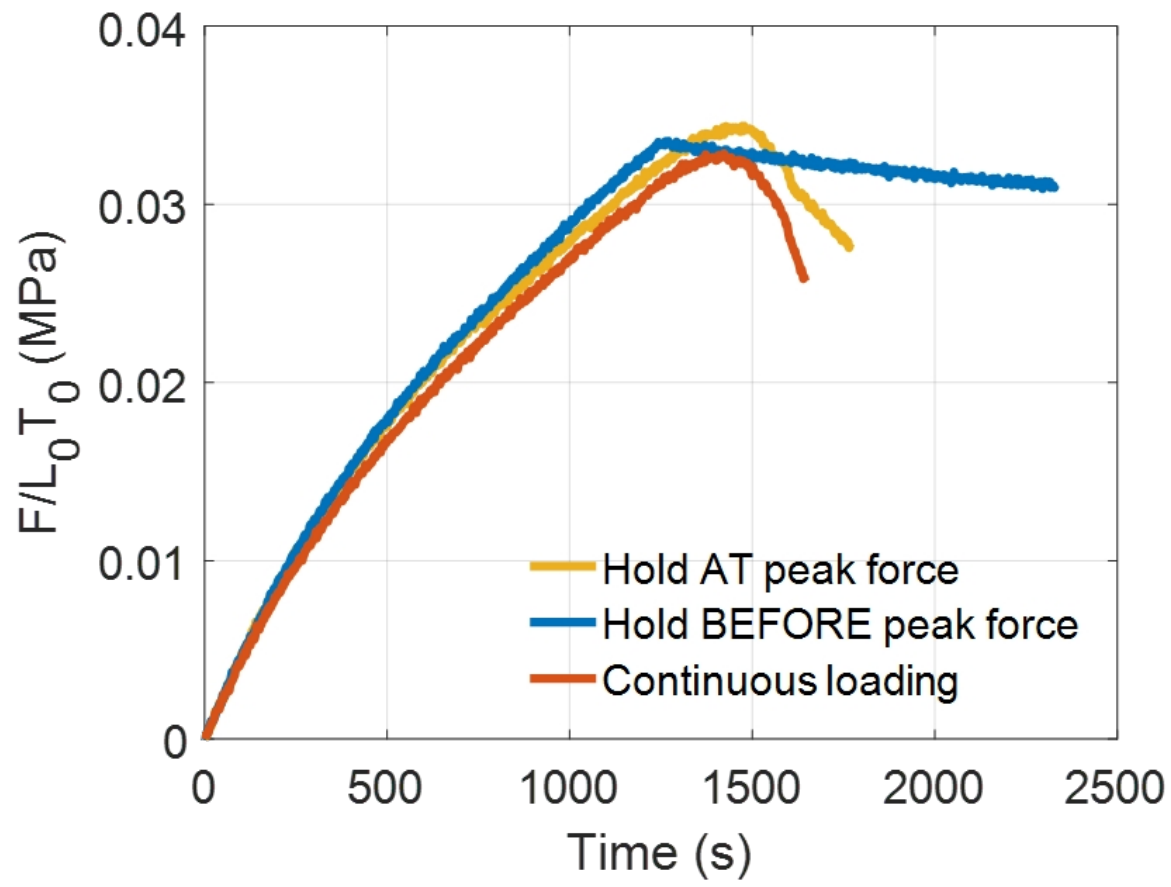



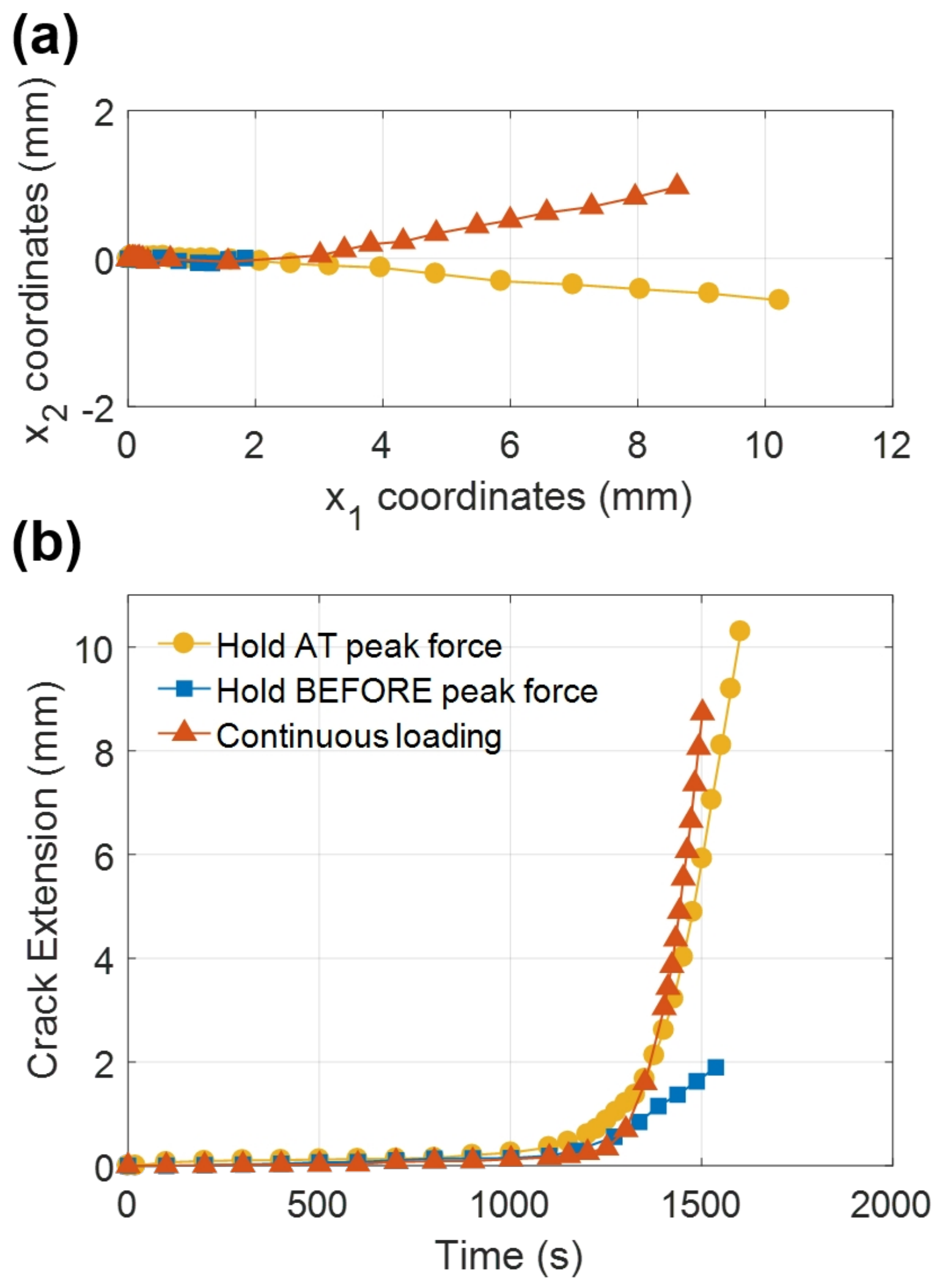
(a)

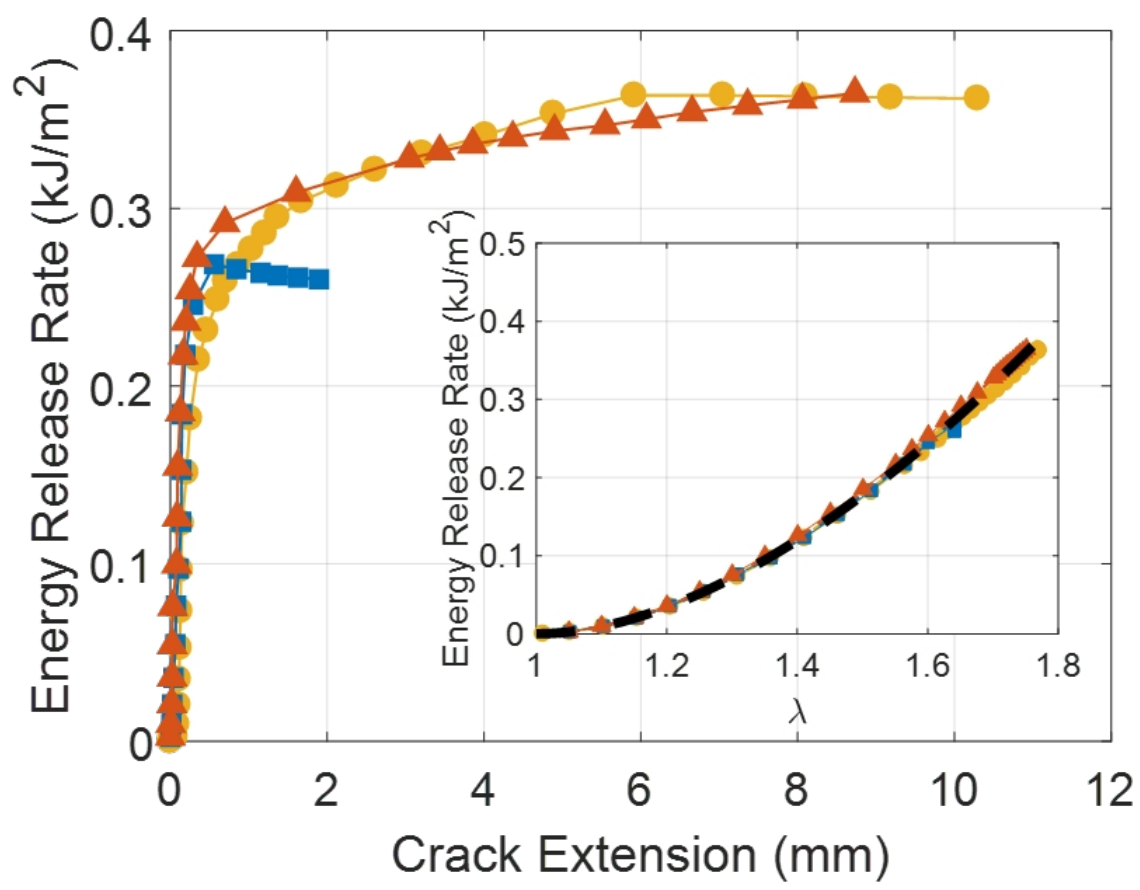

(b)

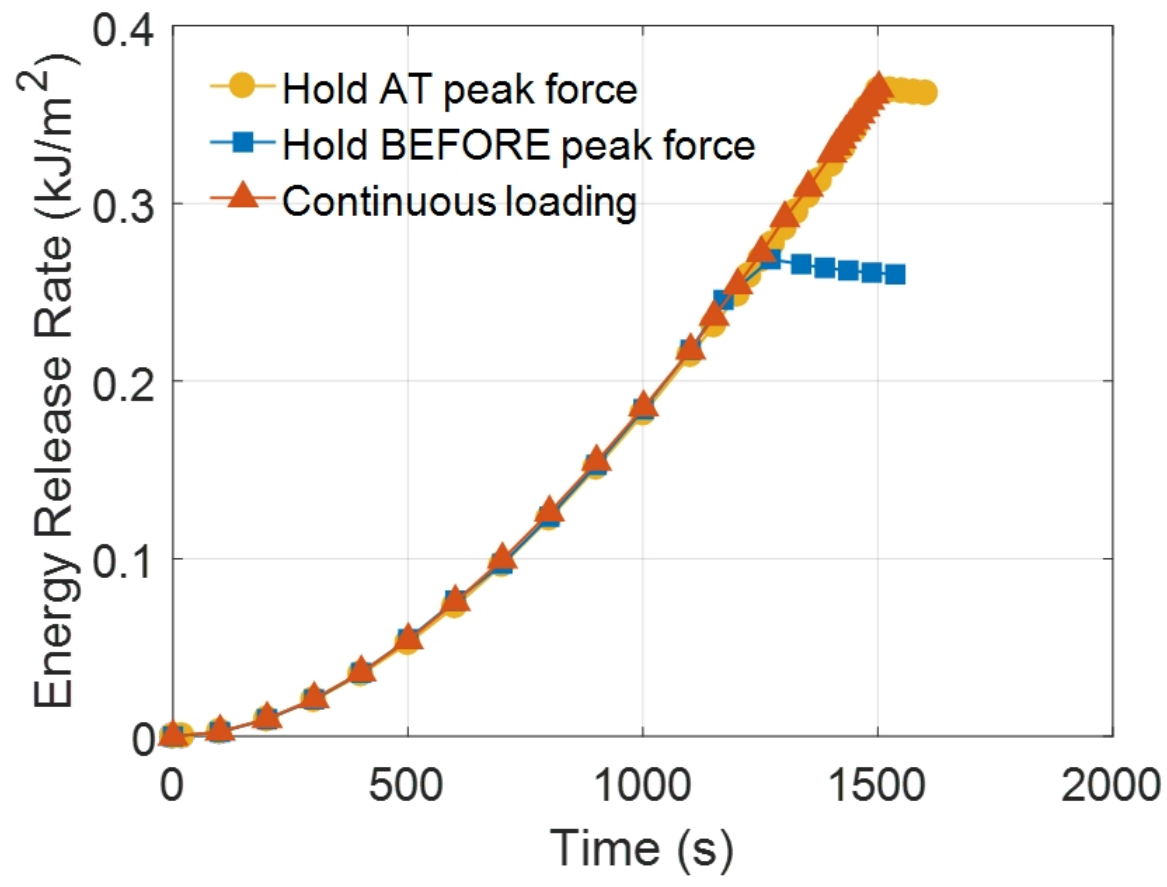




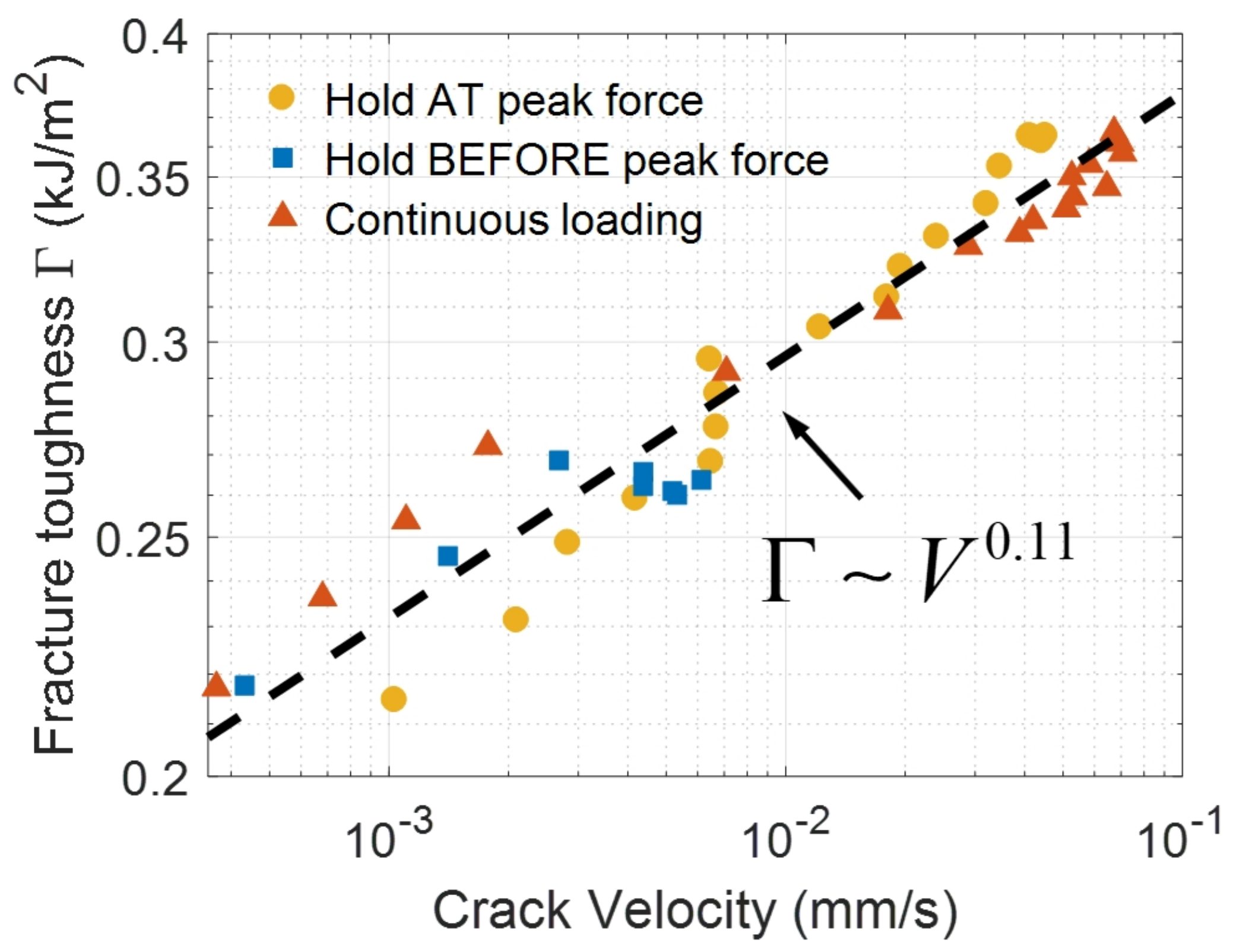


a

UV exposure and develop Photo Resist; etching Copper.

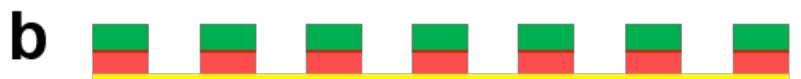

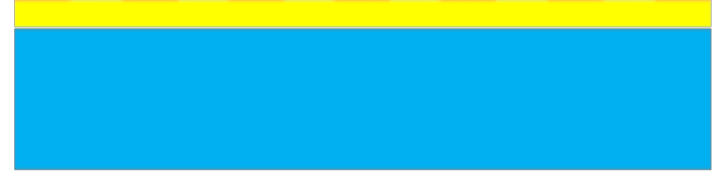

Remove Photo Resist and applied Water soluble tape.

C

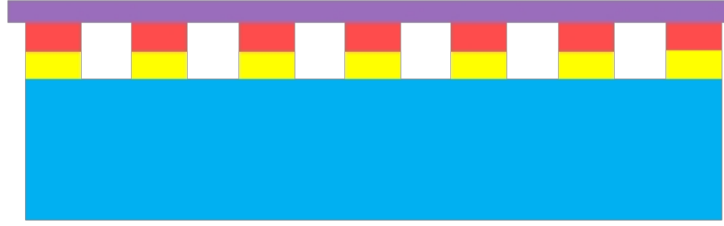

f

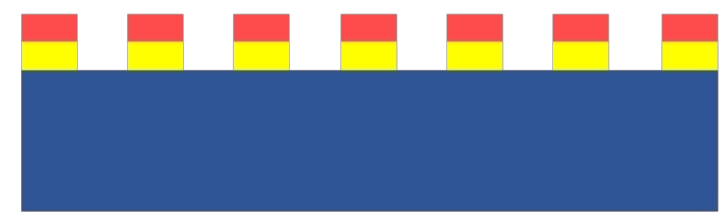

Dissolve Water soluble tape using DI water.

e

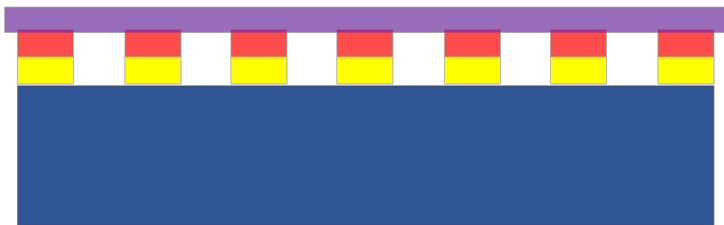

Transfer patterns on Ecoflex substrate using liquid Ecoflex as adhesive.

d Quickly peel off, and transfer patterns to the tape.

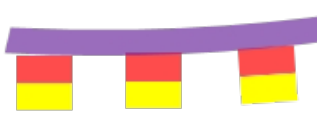

AZ 4210 Photo resist

- Copper $100 \mathrm{~nm}$

Polyimide 1 um

- Glass sheet (PDMS coated)
- Water soluble tape 
a

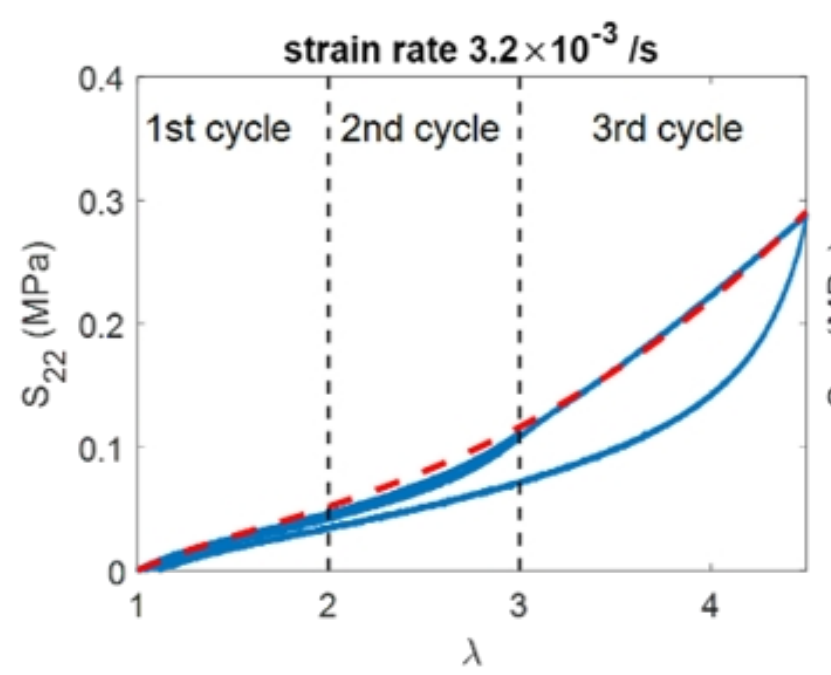

b

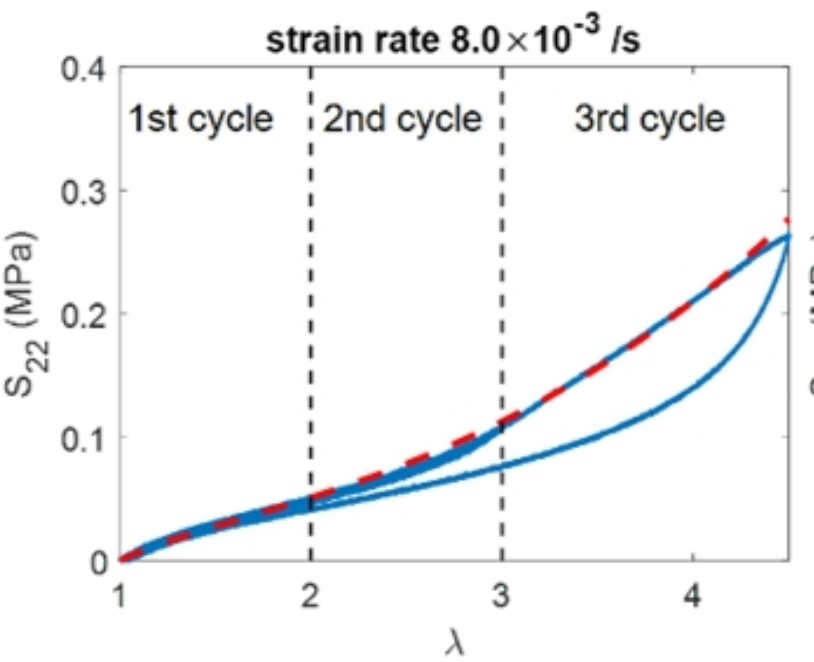

C

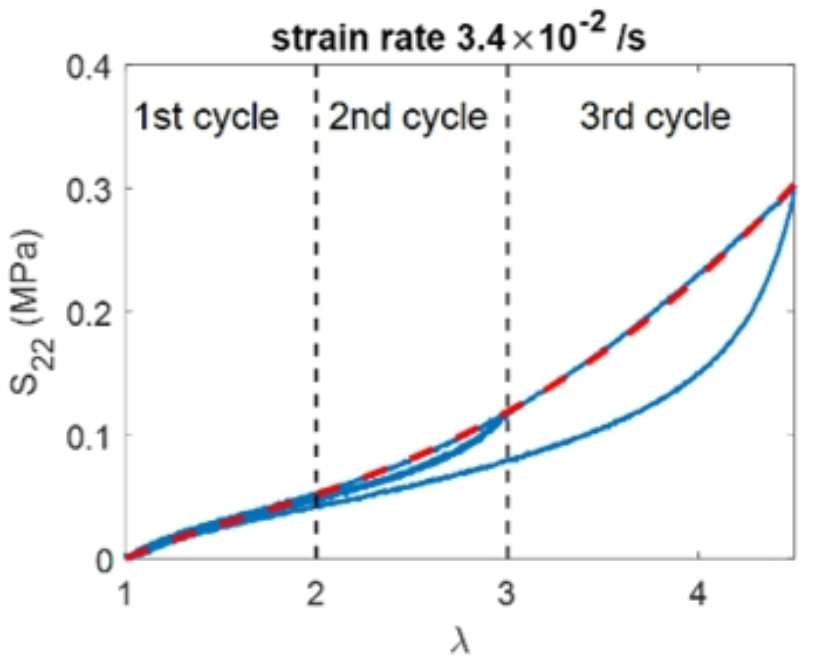

